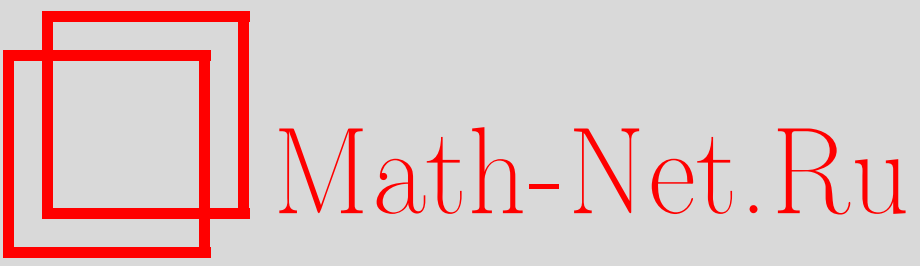

В. В. Вершинин, Группы кос и пространства петель, УМН, 1999, том 54, выпуск 2, 3-84

DOI: https://doi.org/10.4213/rm132

Использование Общероссийского математического портала Math-Net.Ru подразумевает, что вы прочитали и согласны с пользовательским соглашением

http://www.mathnet.ru/rus/agreement

Параметры загрузки:

IP : 54.237 .206 .68

26 апреля 2023 г., 11:03:14 


\title{
ГРУППЫ КОС И ПРОСТРАНСТВА ПЕТЕЛЬ
}

\author{
В. В. ВЕРшинин
}

\section{СОДЕРЖАНИЕ}

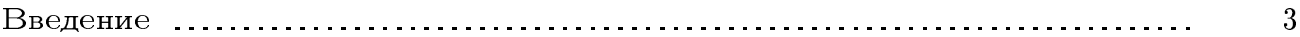

$\S 1$. Группы кос и конфигурационные пространства ................. 6

$\S 2$. Группы автоморфизмов свободных групп ....................... 9

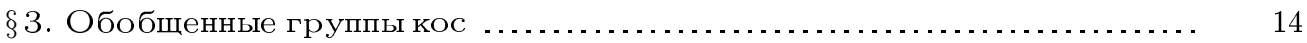

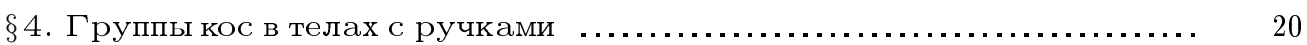

$\S 5$. Косы с особенностями ......................................... 26

$\S 6$. Классифицирующие пространства для групп автоморфизмов свободных групп ............................................ 31

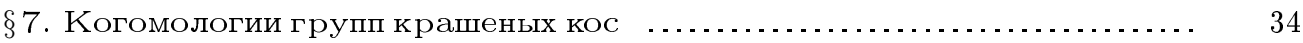

$\S 8$. Гомологии групп кос и пространства петель ....................... 35

$\S 9$. Когомологии обобшенных групп кос ......................... 42

$\S 10$. Порядковые комплексы и когомологии исключительных обобщен-

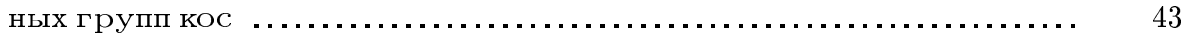

$\S 11$. Гомологии групп кос в телах с ручками и гомологии конфигурационных пространств для поверхностей ....................... 47

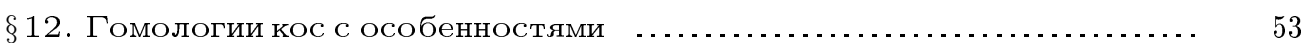

$\S 13$. Косы и спектры Тома ....................................... 62

$\S 14$. Косы, монополи и пространства рациональных функций .......... 71

Дополнение. Категории и пространства петель ....................... 75

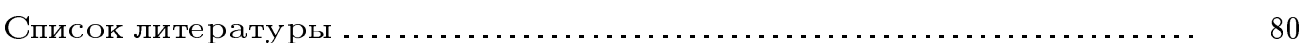

\section{Введение}

Косы были строго определены Э. Артином [1] в 1925 году, хотя корни этого естественного понятия видны в работах А. Гурвица ([2], 1891 г.), Р. Фрике и Ф. Клейна ([3], 1897 г.) и даже в записных книжках К.-Ф. Гаусса. В своей работе [4] Э. Артин дал копредставление группы кос (см. формулы (1.2) в $\S 1)$, широко известное в настояшее время.

Работа выполнена при финансовой поддержке INTAS. 
Косы можно рассматривать с различных точек зрения. Во-первых, они естественно возникают как классы изотопии набора из $n$ связных кривых (нитей) в трехмерном пространстве. С этой точки зрения они принадлежат маломерной топологии. По теореме А. А. Маркова [5], [6] они являются существенными компонентами при изучении зацеплений и узлов. Начиная с работы В.Ф.Р. Джонса [7], обширное семейство инвариантов зацеплений было построено с использованием кос (см., например, [8], [9]). Эта точка зрения связана с определением групшы кос как фундаментальной группы конфигурационного пространства $n$ точек на плоскости [10]. Оказалось, что это пространство является $K(\pi, 1)$-пространством [11] и, таким образом, может служить естественной геометрической моделью классифицируюшего пространства групшы кос. С другой стороны, В.И. Арнольд [12] интерпретировал это конфигурационное пространство как пространство полиномов над $\mathbb{C}$ степени $n$ без кратных корней, т.е. как дополнение в $\mathbb{C}^{n} \mathrm{\kappa}$ дискриминантному множеству. Этот подход также имел последствия для маломерной топологии. А именно, первоначальное определение инвариантов Васильева узлов [13] моделировало ситуацию изучения дополнения к дискриминантному множеству, образованному узлами с простыми особенностями (двойньми точками трансверсальных самопересечений).

Конфигурационное пространство можно рассматривать как пространство орбит дополнения комплексификации конфигурации гиперплоскостей, соответствующей групше Кокстера $A_{n-1}=\Sigma_{n}$. Обобшая этот подход на все конечные групшы Кокстера, Э. Брискорн [14] определил так называемые обобщенные группы кос, которые иногда называют также Артиновыми группами, наверное потому, что Э. Артин никогда их не рассматривал.

Другим способом обобщения является рассмотрение групп кос в трехмерных многообразиях, возможно с краем. Простейшим примером являются группы кос в телах с ручками. А.Б. Сосинский [15] был первым, кто их изучал. Такая группа может быть интерпретирована как фундаментальная группа конфигурационного пространства плоскости без $g$ точек, где $g$ есть род тела с ручками. На языке конфигураций гиперплоскостей это соответствует “дискриминантным" конфигурациям, которые связаны с дифференциальным уравнением Книжника-Замолодчикова и изучались В. В. Шехтманом и А. Н. Варченко [16]. Обобщенная группа кос типа $C$ изоморфна группе кос в полнотории и была использована С. Ламбропулу [17] при построении полинома типа Джонса для зацеплений в полнотории. Групшы кос в телах с ручками использовались автором при изучении инвариантов Васильева зацеплений в телах с ручками [18], [19].

Классическая группа кос, так же как и симметрическая группа, является группой автоморфизмов свободной групшы [4], [20]. Эта точка зрения и копредставление группы кос дают связи с теорией груп и, в частности, с теорией свободных групп.

В попытках интерпретировать инварианты Васильева через косы, следуя общей идеологии В.А. Васильева расширять набор основных объектов до объектов с простыми особенностями, было введено понятие кос с особенностями. Соответствующими алгебраическими структурами являются моноид Баеса-Бирман [21], [22] и группа кос-перестановок Р. Фенна, Р. Риманњи и К. Рурка [23], [24]. По-видимому, косы с особенностями также имеют широкие связи с различными разделами математики. Группа кос-перестановок определяется как подгруппа группы автоморфизмов свободной группы и имеет интерпретацию как группа кос со спайками, как их называют 
авторы. Она может быть также интерпретирована как группа автоморфизмов свободного объекта в категории, образованной некоторой алгебраической структурой с одной бинарной операцией (quandle) [24]. Дж. Баес обсуждал в [21] связи моноида кос с особенностями с теорией возмущений Черна-Саймонса и другими вопросами математической физики.

Когомологии классических групп кос появились в работах В. И. Арнольда [12], [25], опубликованных в 1970 году. Они были введены в связи с изучением проблемы представимости алгебраических функций нескольких переменных посредством суперпозиции алгебраических функций меньшего числа переменных. А именно, в размерностях вида $2^{n}-1$ этих когомологий В. И. Арнольд обнаружил препятствия к такой представимости. В упомянутых работах когомологии групп кос обсуждались в широком математическом контексте, а также демонстрировались их связи с различными математическими дисциплинами. В.И. Арнольд доказал три важные теоремы о целочисленных когомологиях групп кос (теоремы 8.1-8.3 настояшей работы) и проделал вычисления в малых размерностях. В.И. Арнольд также описал полностью когомологии груп крашеных кос [26]. Д. Б. Фукс продолжил исследования когомологий классических групп кос и вычислил когомологии по модулю 2 [27].

Независимо от работ В.И. Арнольда и Д. Б. Фукса Ф.Р. Коэн изучал гомологии классических групп кос другими методами и в связи с теорией конфигурационных пространств, пространств петель, операциями Араки-Кудо-Даера-Лашофа и спектрами Тома [28]-[32]. Он вычислил эти гомологии с коэффициентами в $\mathbb{Z}$, а также в $\mathbb{Z} / p$ как модули над алгеброй Стинрода. Аддитивная структура когомологий групп кос с коэффициентами в $\mathbb{Z}$ была также вычислена $\Phi$. В. Вайнштейном [33], которьй использовал методы Д. Б. Фукса. Позже В. В. Горюнов применил эти методы в [34], [35] и выразил когомологии обобщенных групп кос типов $C$ и $D$ в терминах когомологий классических групп кос. Теорема Г. Сегала [36], устанавливающая связи между гомологиями группы кос из бесконечного числа нитей и двукратным пространством петель на двумерной сфере, является аналогом теоремы М. Баррата, С. Придди и Д. Квиллена [37], [38] о связи между гомологиями бесконечной симметрической групшы и бесконечнократньм пространством петель $\Omega^{\infty} S^{\infty}$. Отсюда происходит связь с работами М. Маховальда [39], [40] по теории гомотопий.

Для вещественной конфигурации гиперплоскостей в $\mathbb{C}^{n}$ (т.е. конфигурации, заданной вещественными уравнениями) М. Сальветти построил в [41] клеточный комплекс размерности $n$, гомотопически эквивалентньй дополнению к конфигурации. В [42] он применил эту конструкцию к конфигурации конечной группы Кокстера, заметил, что в этом случае комплекс допускает естественное свободное действие данной группы Кокстера, которое делает указанную вьше гомотопическую эквивалентность эквивариантной. Затем он получил эффективное описание фактор-комплекса этого действия, что дало простой способ вычисления когомологий всех обобщенных групп кос, в том числе исключительных.

Композишия канонического эпиморфизма групшы кос на симметрическую группу $B r_{n} \rightarrow \Sigma_{n}$ и стандартного представления симметрической групшы в ортогональную группу $\Sigma_{n} \rightarrow O_{n}$ порождает спектр Тома $\left\{M B r_{n}\right\}$. Ф. Р. Коэн [30], М. Маховальд [39] и Ш. Буллет [43] доказали, что спектр $\left\{M B r_{n}\right\}$ изоморфен спектру Эйленберга-Маклейна обычных гомологий с коэффишиентами в $\mathbb{Z} / 2$ (см. теорему 13.3 в $\S 13)$.

Другая удивительная связь, обнаруженная Ф. Р. Коэном, Р. Л. Коэном, Б. М. Ман- 
ном, Р. Дж. Мильграмом [44] и С. К. Дональдсоном [45], наблюдается между классифицируюшим пространством группы кос, пространством комплексных рациональных функций и пространством модулей монополей. Используя эту связь, Р. Л. Коэн и Дж. Д. С. Джонс проинтерпретировали индексное расслоение оператора Дирака в гомотопических терминах [46], [47].

Мы не касаемся в нашем обзоре алгоритмических проблем, связанных с косами [48]-[51], потому что теорема Гарсайда отражена в книге Дж. Бирман [6], а более полное обсуждение этих вопросов невозможно из-за существенного увеличения объема настоящей работы. Связи, открытые С. Смейлом [52] и развитые В. А. Васильевым [53], между когомологиями кос и сложностью алгоритмов также не рассматриваются. Этот предмет уже отражен в монографической литературе [13].

Настояший обзор организован следуюшим образом. В первых пяти параграфах мы обсуждаем косы без упоминаний о классифицируюших пространствах или (ко)гомологиях. Эти объекты возникают в $\S 6$ и изучаются в связи с группами кос и пространствами петель в следуюших параграфах. В $\S 1$ мы вводим конфигурационные пространства, фундаментальные группы которых являются группами кос. Связи с группами автоморфизмов свободных групп рассматриваются в $\S 2$. В $\S 3$ мы даем краткий очерк о группах Кокстера и изучаем обобщенные группы кос, которые этими группами Кокстера определяются. Свойства групп кос в телах с ручками обсуждаются в $\S 4$. Косы с особенностями появляются в $\S 5$. В $\S 6$ рассматриваются некоторые обшие черты классифишируюших пространств групп автоморфизмов свободных групп. В $\S 7$ даны когомологии групा крашеных кос. Мы обсуждаем различные аспекты (ко)гомологий классических групп кос в $\S 8$. В $\S 9$ мы описываем когомологии обобшенных групп кос типов $C$ и $D$ в терминах когомологий классических групп кос. Параграф 10 содержит конструкцию комплекса Сальветти и его приложения к когомологиям обобщенных групп кос. Гомологические свойства групп кос в телах с ручками и кос с особенностями изучаются соответственно в $\S 11$ и 12 . Мы изучаем спектры Тома групा кос в $\S 13$. В $\S 14$ указаны связи с пространством рациональных функций комплексного переменного и теорией монополей. В дополнении собраны необходимые понятия и факты из теории категорий и алгебраической топологии.

Автор благодарен В.И. Арнольду, который привлек его внимание к связи между косами и теорией монополей и снабдил необходимой литературой по этому предмету. Особая благодарность В.А. Васильеву за массу полезных замечаний (от принципиальных до опечаток). Автор также признателен $\Phi$. Р. Коэну за плодотворные беседы о когомологиях кос, С.С. Кутателадзе и Е.П. Волокитину за помошь и советы, касаюшиеся изложения материала работы, а также В. М. Бухштаберу, без внимания которого этот обзор никогда бы не был написан.

\section{$\S 1$. Группы кос и конфигурационные пространства}

Косы естественно возникают как объектыв трехмерном пространстве. Чтобы формализовать их как группу, простейшим способом является интерпретировать их как фундаментальную группу конфигурационного пространства.

Пусть $Y$ есть связное топологическое многообразие, а $W$ есть конечная группа, действуюшая на $Y$. Точка $y \in Y$ назьвается регулярной, если ее стабилизатор $\{w \in W$ : $w y=y\}$ тривиален, т.е. состоит только из единицы групшы $W$. Множество $Y$ всех ре- 
гулярных точек открыто. Предположим, что оно связно и непусто. Подпространство $\operatorname{ORB}(Y, W)$ пространства всех орбит $\operatorname{Orb}(Y, W)$, состоящее из орбит всех регулярных точек, назьвается пространством регулярных орбит. Имеется свободное действие $W$ на $\widetilde{Y}$, и проекция $p: \widetilde{Y} \rightarrow \widetilde{Y} / W=O R B(Y, W)$ определяет накрытие. Рассмотрим начальньй отрезок длинной точной последовательности этого накрытия:

$$
1 \longrightarrow \pi_{1}\left(\widetilde{Y}, y_{0}\right) \stackrel{p_{*}}{\longrightarrow} \pi_{1}\left(\operatorname{ORB}(Y, W), p\left(y_{0}\right)\right) \longrightarrow W \longrightarrow 1 .
$$

Фундаментальная группа $\pi_{1}\left(O R B(Y, W), p\left(y_{0}\right)\right)$ пространства регулярных орбит назьвается группой кос действия $W$ на $Y$ и обозначается через $\operatorname{Br}(Y, W)$. Фундаментальная группа $\pi_{1}\left(\widetilde{Y}, y_{0}\right)$ назьвается группой крашеных кос действия $W$ на $Y$ и обозначается через $P(Y, W)$. Пространства $\widetilde{Y}$ и $O R B(Y, W)$ линейно связны, так что обе эти группы определены однозначно с точностью до изоморфизма и можно не указьвать в обозначениях базисную точку $y_{0}$.

Для любого пространства $Y$ симметрическая группа $\Sigma_{m}$ действует на декартовой степени $Y^{m}$ пространства $Y$ :

$$
w\left(y_{1}, \ldots, y_{m}\right)=\left(y_{w^{-1}(1)}, \ldots, y_{w^{-1}(m)}\right), \quad w \in \Sigma_{m} .
$$

Обозначим через $F(Y, m)$ пространство $m$-ок попарно различных точек в $Y$ :

$$
F(Y, m)=\left\{\left(p_{1}, \ldots, p_{m}\right) \in Y^{m}: p_{i} \neq p_{j} \text { при } i \neq j\right\} .
$$

Оно является пространством регулярных точек указанного действия. В случае, когда $Y$ есть связное топологическое многообразие $M$ без края и $\operatorname{dim} M \geqslant 2$, пространство регулярных орбит $O R B\left(M^{m}, \Sigma_{m}\right)$ открыто, связно и непусто. Мы называем ORB $\left(M^{m}, \Sigma_{m}\right)$ конфигурачионныц пространством многообразия $M$ и обозначаем $B(M, m)$. Группа кос $B r\left(M^{m}, \Sigma_{m}\right)$ называется группой кос из $m$ нитей многообразия $M$ и обозначается $\operatorname{Br}(m, M)$. Аналогично мы называем группу $P\left(M^{m}, \Sigma_{m}\right)$ группой крашеных кос из $m$ нитей многообразия $M$ и обозначаем $P(m, M)$. Эти определения групп кос были даны Р. Фоксом и Л. Нойвиртом [10]. Классическая группа кос из $m$ нитей $B r_{m}$ и соответствуюшая группа крашеных кос $P_{m}$ возникают в случае, когда многообразие $M$ является евклидовой плоскостью $\mathbb{R}^{2}$.

Пусть $\left(q_{i}\right)_{i \in \mathbb{N}}$ есть фиксированная последовательность различных точек многообразия $M$, положим $Q_{m}=\left\{q_{1}, \ldots, q_{m}\right\}$. Мы будем использовать

$$
Q_{m, l}=\left(q_{l+1}, \ldots, q_{l+m}\right) \in F\left(M \backslash Q_{l}, m\right)
$$

в качестве стандартной базисной точки пространства $F\left(M \backslash Q_{l}, m\right)$. Если $k<m$, определим проекцию

$$
\operatorname{proj}: F\left(M \backslash Q_{l}, m\right) \rightarrow F\left(M \backslash Q_{l}, k\right)
$$

по формуле: $\operatorname{proj}\left(p_{1}, \ldots, p_{m}\right)=\left(p_{1}, \ldots, p_{k}\right)$. Следуюшие теоремы были доказаны Э. Фаделлом и Л. Нойвиртом [11]. 
Tеорема 1.1. Тройка proj: $F\left(M \backslash Q_{l}, m\right) \rightarrow F\left(M \backslash Q_{l}, k\right)$ является локально тривиальным расслоением со слоем $\operatorname{proj}^{-1} Q_{k, l}$, гомеоморфным $F\left(M \backslash Q_{k+l}, m-k\right)$.

Из рассмотрения последовательности расслоений

$$
\begin{aligned}
& F\left(M \backslash Q_{m-1}, 1\right) \rightarrow F\left(M \backslash Q_{m-2}, 2\right) \rightarrow M \backslash Q_{m-2}, \\
& F\left(M \backslash Q_{m-2}, 2\right) \rightarrow F\left(M \backslash Q_{m-3}, 3\right) \rightarrow M \backslash Q_{m-3}, \\
& F\left(M \backslash Q_{1}, m-1\right) \rightarrow F(M, m) \rightarrow M
\end{aligned}
$$

вытекает следующий факт.

ТЕОремА 1.2. Для любого многообразия $M$

$$
\pi_{i}\left(F\left(M \backslash Q_{1}, m-1\right)\right)=\bigoplus_{k=1}^{m-1} \pi_{i}\left(M \backslash Q_{k}\right)
$$

при $i \geqslant 2$. Если proj: $F(M, m) \rightarrow M$ допускает сечение, то

$$
\operatorname{proj}_{i} \pi_{i}(F(M, m))=\bigoplus_{k=0}^{m-1} \pi_{i}\left(M \backslash Q_{k}\right), \quad i \geqslant 2 .
$$

СлЕДСТВИЕ 1.1. Если $М$ есть г-мерное евклидово пространство, то

$$
\pi_{i}(F(M, m))=\bigoplus_{k=0}^{m-1} \pi_{i}(\underbrace{S^{r-1} \vee \cdots \vee S^{r-1}}_{k}), \quad i \geqslant 2 .
$$

СлЕДСТВИЕ 1.2. Если $M$ есть двумерное евклидово пространство, то $F\left(\mathbb{R}^{2}, m\right)$ есть $K\left(P_{m}, 1\right)$-пространство, a $B\left(\mathbb{R}^{2}, m\right)$ есть $K\left(B r_{m}, 1\right)$-nространство.

В этом случае точная последовательность (1.1) имеет следующий вид:

$$
1 \longrightarrow P_{m} \longrightarrow B r_{m} \stackrel{\tau_{m}}{\longrightarrow} \Sigma_{m} \longrightarrow 1
$$

Она может быть использована для доказательства канонического копредставления групшы кос $B r_{m}$ с образуюшими $\sigma_{i}, i=1, \ldots, m-1$, и соотношениями:

$$
\left\{\begin{array}{l}
\sigma_{i} \sigma_{j}=\sigma_{j} \sigma_{i}, \text { если }|i-j|>1 \\
\sigma_{i} \sigma_{i+1} \sigma_{i}=\sigma_{i+1} \sigma_{i} \sigma_{i+1}
\end{array}\right.
$$

Детали можно найти, например, в книге Дж. Бирман [6].

При интерпретации кос как классов изотопии набора из $n$ связных кривых (нитей) в трехмерном пространстве образуюшая $\sigma_{i}$ соответствует набору кривых, изображенному на рис. 1.1. 


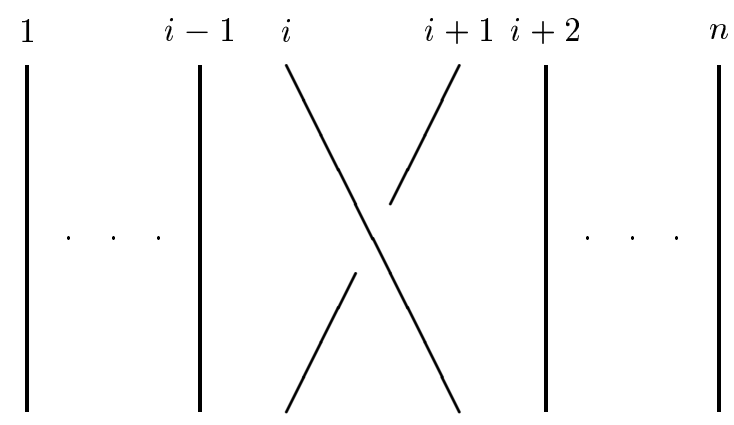

Рис. 1.1

Конечно, имеются и другие копредставления группы кос. Интересная серия копредставлений была дана В. Сержиеску [54]. Для каждого плоского графа он построил копредставление группы $B r_{n}$, где $n$ есть число вершин графа, образуюшие соответствуют ребрам, а соотношения отражают геометрию графа. Представление Артина в этом контексте соответствует графу, состоящему из отрезка вещественной прямой от 1 до $n$ с натуральными числами (от 1 до $n$ ) в качестве вершин и отрезками, их соединяюшими, в качестве ребер.

\section{§2. Группы автоморфизмов свободных групп}

Другой важный подход к группе кос основывается на факте, что она может рассматриваться как подгруппа группы всех автоморфизмов свободной группы.

Пусть $F_{n}$ есть свободная группа ранга $n$ с множеством свободных образуюших $\left\{x_{1}, \ldots, x_{n}\right\}$. Предположим далее, что Aut $F_{n}$ есть группа автоморфизмов группы $F_{n}$. Имеем стандартные вложения симметрической групाы $\Sigma_{n}$ и группы кос $B r_{n}$ в группу Aut $F_{n}$. Они могут быть описаны следуюшим образом. Пусть $\xi_{i} \in \operatorname{Aut} F_{n}$, $i=1,2, \ldots, n-1$, заданы следуюшими формулами действия на образуюших:

$$
\left\{\begin{aligned}
x_{i} & \mapsto x_{i+1}, \\
x_{i+1} & \mapsto x_{i}, \\
x_{j} & \mapsto x_{j}, \quad j \neq i, i+1,
\end{aligned}\right.
$$

а автоморфизмы $\sigma_{i} \in \operatorname{Aut} F_{n}, i=1,2, \ldots, n-1$, определяются формулами:

$$
\left\{\begin{aligned}
x_{i} & \mapsto x_{i+1}, \\
x_{i+1} & \mapsto x_{i+1}^{-1} x_{i} x_{i+1}, \\
x_{j} & \mapsto x_{j}, \quad j \neq i, i+1 .
\end{aligned}\right.
$$

Свободная группа $F_{n}$ является фундаментальной группой диска $D_{n}$ без $n$ точек, а образуюшая $x_{i}$ соответствует петле, обходящей вокруг $i$-й точки. Группа кос $B r_{n}$ является группой классов отображений диска $D_{n}$ с фиксированным краем [6] и действует на фундаментальной групше $D_{n}$ по формулам $(2.2)$. Геометрически это действие изображено на рис. 2.1 . 

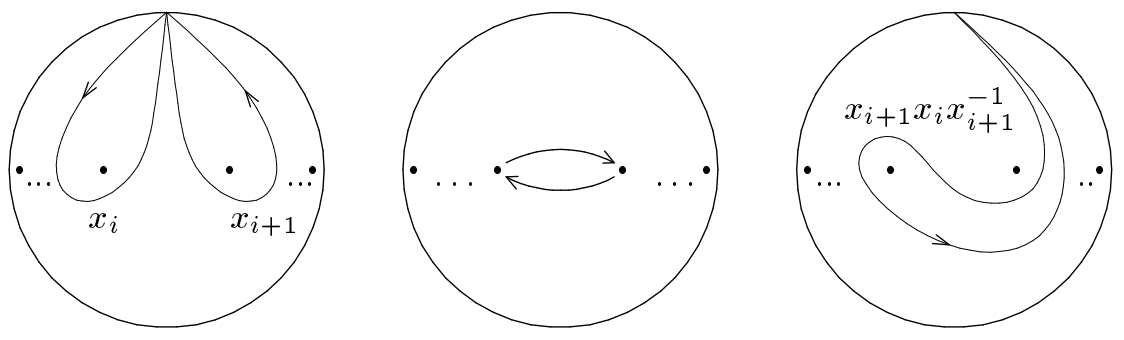

Рис. 2.1

Отображая стандартные образуюшие симметрической группы на $\xi_{i}$, а стандартные образуюшие группы кос на $\sigma_{i}$, мы получим мономорфизмы групा $r$ и $t$ :

$$
\begin{aligned}
t: \Sigma_{n} & \rightarrow \text { Aut } F_{n}, \\
r: B r_{n} & \rightarrow \operatorname{Aut} F_{n} .
\end{aligned}
$$

Используя такое определение групшы кос, А. А. Марков [20] доказал копредставление (1.2).

Имеем также канонические вложения

$$
\text { Aut } F_{m} \times \text { Aut } F_{n} \rightarrow \text { Aut } F_{m+n},
$$

согласуюшиеся со стандартными спариваниями симметрических групп и групा кос

$$
\begin{gathered}
\Sigma_{k} \times \Sigma_{l} \rightarrow \Sigma_{k+l}, \\
\mu: B r_{k} \times B r_{l} \rightarrow B r_{k+l} .
\end{gathered}
$$

Эти спаривания коммутируют с отображениями $\tau_{j}: B r_{j} \rightarrow \Sigma_{j}$. Для групп кос это спаривание может быть построено добавлением дополнительных $l$ нитей к начальным $k$. Если $\sigma_{i}^{\prime}$ суть образуюшие $B r_{k}, \sigma_{j}^{\prime \prime}$ - образуюшие $B r_{l}$ и $\sigma_{r}$ - образуюшие $B r_{k+l}$, тогда отображение $\mu$ может быть выражено в виде

$$
\begin{array}{cl}
\mu\left(\sigma_{i}^{\prime}, e\right)=\sigma_{i}, & 1 \leqslant i \leqslant k-1, \\
\mu\left(e, \sigma_{j}^{\prime \prime}\right)=\sigma_{j+k}, & 1 \leqslant j \leqslant l-1 .
\end{array}
$$

Пусть $f\left(y_{1}, \ldots, y_{m}\right)$ есть слово с вхождениями (возможно, пустыми) $y_{i}^{\varepsilon}$, где $y_{i}-$ некоторые буквы, а $\varepsilon$ может быть \pm 1 . Если $y_{i}$ являются элементами группы $G$, тогда $f\left(y_{1}, \ldots, y_{m}\right)$ будет рассматриваться как элемент $G$.

Определим элементы $s_{i, j}, 1 \leqslant i<j \leqslant m$, группы кос $B r_{m}$ по формуле:

$$
s_{i, j}=\sigma_{j-1} \ldots \sigma_{i+1} \sigma_{i}^{2} \sigma_{i+1}^{-1} \ldots \sigma_{j-1}^{-1} .
$$

Эти элементы удовлетворяют следуюшим соотношениям Бурау ([20], см. также [55]):

$$
\begin{array}{rlrl}
s_{i, j} s_{k, l} & =s_{k, l} s_{i, j} & & \text { для } i<j<k<l \text { и } i<k<l<j, \\
s_{i, j} s_{i, k} s_{j, k} & =s_{i, k} s_{j, k} s_{i, j} & & \text { для } i<j<k, \\
s_{i, k} s_{j, k} s_{i, j} & =s_{j, k} s_{i, j} s_{i, k} & & \text { для } i<j<k, \\
s_{i, k} s_{j, k} s_{j, l} s_{j, k}^{-1} & =s_{j, k} s_{j, l} s_{j, k}^{-1} s_{i, k} & \text { для } i<j<k<l .
\end{array}
$$


А. А. Марков доказал, что элементы $s_{i, j}$ вместе с соотношениями (2.5) дают копредставление группы крашенных кос $P_{m}[20]$. Следуюшая формула является следствием соотношений Бурау и также принадлежит А. А. Маркову:

$$
\left[s_{i, l}, s_{j, k}^{\varepsilon}\right]=f\left(s_{1, l}, \ldots, s_{l-1, l}\right), \quad \varepsilon= \pm 1, \quad k<l .
$$

Определим элементы $\sigma_{k, l}, 1 \leqslant k \leqslant l \leqslant m$, по формулам:

$$
\begin{aligned}
\sigma_{k, k} & =e, \\
\sigma_{k, l} & =\sigma_{k} \ldots \sigma_{l-1} .
\end{aligned}
$$

ЗАмЕчАнИЕ. Наше определение $\sigma_{k, l}$ отличается от первоначального, данного А. А. Марковым:

$$
\sigma_{k, l}=\sigma_{k}^{-1} \ldots \sigma_{l-1}^{-1} .
$$

Пусть $P_{m}^{k}$ есть подгруппа $P_{m}$, порожденная элементами $s_{i, j}$ с $k<j$.

Tеорема 2.1 (А.А. Марков). (i) Каждый әлемент группь $B r_{m}$ может быть единственным образом записан в виде

$$
f_{2}\left(s_{1,2}\right) \ldots f_{j}\left(s_{1, j}, \ldots, s_{j-1, j}\right) \ldots f_{m}\left(s_{1, m}, \ldots, s_{m-1, m}\right) \sigma_{i_{m}, m} \ldots \sigma_{i_{j}, j} \ldots \sigma_{i_{2}, 2} .
$$

(ii) Фактор группа $P_{m}^{k} / P_{m}^{k-1}$ есть свободная группа от свободных образующих $s_{i, k+1}, 1 \leqslant i \leqslant k$.

ЗАМЕчАнИЕ. В копредставлении (2.7) наш порядок элементов $f_{j}$ противоположен первоначальному, определенному А. А. Марковым. Доказательство то же самое, что и в [20]: используется формула коммутирования (2.6).

Пусть теперь $G_{n}, n=0,1, \ldots, G_{0}=\{e\}$, есть система подгрупп групп автоморфизмов свободных групп:

$$
G_{n} \leqslant \text { Aut } F_{n}
$$

такая, что $G_{m+n}$, как подгруппа Aut $F_{m+n}$, содержит образ $G_{m} \times G_{n}$ при гомоморфизме (2.3) для всех $m$ и $n$. В этом случае мы имеем систему гомоморфизмов

$$
\mu_{m, n}(G): G_{m} \times G_{n} \rightarrow G_{m+n} .
$$

Эти спаривания являются строго ассоциативными, что влечет коммутативность диаграммы

$$
\begin{array}{cr}
G_{l} \times G_{m} \times G_{n} & \stackrel{\operatorname{Id} \times \mu_{m, n}}{\longrightarrow} G_{l} \times G_{m+n} \\
\downarrow \mu_{l, m} \times \mathrm{Id} & { }^{\mu_{l, m+n}} \\
G_{l+m} \times G_{n} & \stackrel{\mu_{l+m, n}}{\longrightarrow} G_{l+m+n}
\end{array}
$$

Предположим теперь, что для каждого $m$ группа $G_{m}$ содержит симметрическую группу $\Sigma_{m}$, канонически вложенную в Aut $F_{m}$. В этом случае обычньм образом определяется строгая моноидальная (тензорная) категория $\mathscr{G}$. А именно, ее объекты $\{\overline{0}, \overline{1}, \ldots\}$ 
соответствуют целым числам от 0 до бесконечности, а морфизмы определяются по формуле

$$
\operatorname{hom}(\bar{k}, \bar{l})= \begin{cases}G_{k}, & \text { если } k=l \\ \varnothing, & \text { если } k \neq l\end{cases}
$$

Категория $\Sigma$, соответствуюшая симметрическим группам, имеет симметрию (см. [56; с. 180], а также дополнение к настоящему обзору), т.е. систему изоморфизмов

$$
s \bar{m}, \bar{n}: \overline{m+n} \cong \overline{n+m}
$$

естественную по отношению к морфизмам из $\bar{m}$ в себя и из $\bar{n}$ в себя, обладающую некоторым свойством когерентности и такую, что

$$
s_{\bar{m}, \bar{n}} s_{\bar{n}, \bar{m}}=1 \overline{m+n} .
$$

ПРЕДЛОЖЕНИЕ 2.1. Если группа $G_{m}, m=0,1, \ldots$, cодержит симметрическую группу $\Sigma_{m}$ для всех $m$, то симметрия из категории, порохденной симметрическими группами, индуцирует симметрию в категории Ч. Таким образом, последняя становится пермутативной категорией, а функтор, индуцированный вложениями $\Sigma_{m} \rightarrow G_{m}$, становится морфизмом между пермутативными категориями.

Предположим, что мы имеем систему нормальных подгрупп $N_{m} \triangleleft G_{m}$ для всех $m$, такую, что $N_{m+n}$, как подгруппа Aut $F_{m+n}$, содержит $N_{m} \times N_{n}$ для всех $m$ и $n$. Пусть $H_{m}$ есть система фактор-групп $H_{m}=G_{m} / N_{m}$ и $\mathscr{H}$ есть соответствуюшая категория.

С ЛЕДСТВИЕ 2.1. Категория $\mathscr{H}$ является пермутативной категорией, а функтор $\mathscr{G} \rightarrow \mathscr{H}$, индуиированньй эпиморфизмами $G_{m} \rightarrow H_{m}$, является морфизмом между пермутативными категориями.

Имеются еще такие мультипликативные структуры для систем симметрических групп и групп кос:

$$
\begin{aligned}
\Sigma_{k} \times \Sigma_{l} & \rightarrow \Sigma_{k l}, \\
B r_{k} \times B r_{l} & \rightarrow B r_{k l} .
\end{aligned}
$$

Для системы симметрических групп это спаривание может быть описано следующим образом. Пусть $\sigma \in \Sigma_{k}$ и $s \in \Sigma_{l}$. Тогда $\sigma \circ s \in \Sigma_{k l}$ задается по формуле

$$
\sigma \circ s:(i-1) k+j \mapsto(s(i)-1) k+\sigma(j) \text { для } 1 \leqslant i \leqslant l, 1 \leqslant j \leqslant k .
$$

Это означает, что $\sigma$ о $s$ переставляет $k l$ символов, разделенных на $l$ блоков (по $k$ символов в каждом), таким образом, что $\sigma \circ s$ переставляет блоки как перестановка $s$ и $\sigma \circ s$ переставляет символы внутри каждого блока как перестановка $\sigma$. Спаривание (2.11) для групп кос описывается следуюшим образом. Мы утолшаем каждую нить косы 
$\beta \in B r_{l}$ и вкладываем косу $\alpha \in B r_{k}$ в каждую получившуюся трубку. Графически это изображено на рис. 2.2 .

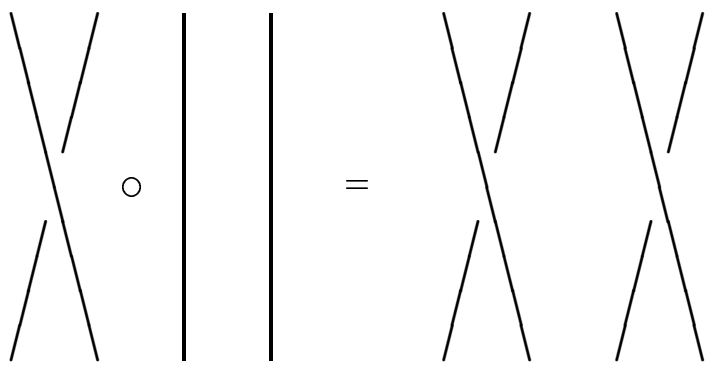

Рис. 2.2

Пусть $\alpha \in$ Aut $F_{k}$ и $\beta \in$ Aut $F_{l}$ суть автоморфизмы свободных групп от образуюших $x_{1}, \ldots, x_{k}$ и $y_{1}, \ldots, y_{l}$. Распишем действие автоморфизмов $\alpha$ и $\beta$ на $x_{j}$ и $y_{i}$ :

$$
\begin{aligned}
& \alpha: x_{j} \mapsto \omega_{j}\left(x_{1}, \ldots, x_{k}\right), \\
& \beta: y_{i} \mapsto \nu_{i}\left(y_{1}, \ldots, y_{l}\right) .
\end{aligned}
$$

Пусть $F_{k l}$ есть свободная группа от образуюших $a_{i, j}$, где $1 \leqslant i \leqslant l, 1 \leqslant j \leqslant k$. Определим эндоморфизм $\alpha \circ \beta$ группы $F_{k l}$ по формуле

$$
\alpha \circ \beta: a_{i, j} \mapsto \nu_{i}\left(\omega_{j}\left(a_{1,1}, \ldots, a_{1, k}\right), \ldots, \omega_{j}\left(a_{l, 1}, \ldots, a_{l, k}\right)\right) .
$$

Рассмотрим группу Aut $F_{k}$ как $\pi_{0} H$-пространства гомотопических самоэквивалентностей букета из $k$ окружностей $\vee^{k} S^{1}:$ Aut $\left(\vee^{k} S^{1}\right)$. Группа Aut $F_{k}$ гомотопически эквивалентна $\operatorname{Aut}\left(\vee^{k} S^{1}\right)$. Тогда элемент $\alpha \circ \beta$ может быть описан следуюшим образом. Пусть $\beta$ представлен отображением букета из $l$ окружностей:

$$
b: \vee^{l} S^{1} \rightarrow \vee^{l} S^{1} .
$$

Отметим точки $p_{i, j}, 1 \leqslant i \leqslant l, 0 \leqslant j \leqslant k$, которые делят $i$-ю окружность на $k$ равных частей так, что $p_{i, 0}$ является базисной точкой букета. Мы берем такой представитель $b$ элемента $\beta$, что $b\left(p_{i, j}\right)$ есть одна из точек $p_{i, j}, 1 \leqslant i \leqslant l, 1 \leqslant j \leqslant k$. Это можно сделать, например, следуюшим образом. Рассмотрим окружность, соответствуюшую символу $y_{i}$, и пусть $d_{i}$ есть длина слова $\nu_{i}\left(y_{1}, \ldots, y_{l}\right)$. Мы отображаем интервал $[0,1]$ линейно на интервал $\left[0, d_{i}\right]$. Тогда точки $r / k, r=0,1, \ldots, k$, отобразятся на точки $d_{i} r / k$. Факторизуя по целочисленной решетке, мы получим нужньй представитель элемента $\beta$. Это отображение $b$ определяет гомотопическую эквивалентность букета из $k l$ окружностей на себя

$$
b: \vee^{k l} S^{1} \rightarrow \vee^{k l} S^{1} .
$$

Букет $\vee^{k l} S^{1}$ естественно разделен на $l$ букетов, имеюших $k$ окружностей в каждом. Применим теперь гомотопическую эквивалентность, соответствующую $\alpha$, на каждом таком подбукете. Мы получим гомотопическую эквивалентность, соответствуюшую 
$\alpha \circ \beta$. Таким образом, $\alpha \circ \beta$, определенньй исходя из (2.12), является автоморфизмом группы $F_{k l}$. Это определяет спаривание

$$
\text { Aut } F_{k} \times \text { Aut } F_{l} \rightarrow \text { Aut } F_{k l}
$$

согласованное с (2.10) и (2.11). Оно ассоциативно. Согласованность с (2.10) видна, если мы будем интерпретировать симметрическую группу таким же образом, как и Aut $F_{k}$, заменив $S^{1}$ на $S^{0}$. Отображение $\operatorname{Aut}\left(\vee^{k} S^{0}\right) \rightarrow \operatorname{Aut}\left(\vee^{k} S^{1}\right)$ индуцировано функтором надстройки.

\section{§3. Обобшенные группы кос}

Группы кос входят в серию так называемых обобщенных групп кос (или Артиновых групп). Нам потребуются некоторые предварительные рассмотрения для определения этих групп.

Пусть $V$ есть конечномерное вещественное векторное пространство $(\operatorname{dim} V=n)$ с евклидовой структурой. Пусть $W$ есть конечная подгруппа $G L(V)$, порожденная отражениями. Мы используем терминологию и обозначения из книги Н. Бурбаки [57]. Пусть $\mathscr{M}$ есть множество гиперплоскостей таких, что $W$ порождена ортогональными отражениями относительно $M \in \mathscr{M}$. Мы предполагаем, что для любого $w \in W$ и любой гиперплоскости $M \in \mathscr{M}$ гиперплоскость $w(M)$ принадлежит $\mathscr{M}$. Гиперплоскости системы $\mathscr{M}$ делят все пространство $V$ (включая сами гиперплоскости) на клетки, назьваемые ячейками. Ячейки максимальной размерности (равной $n$ ) назьваются камерами. Граница камеры $A$ является подмножеством некоторого объединения гиперплоскостей. Эти гиперплоскости называются стенками камеры А. Следующие факты хорошо известны [57].

ПРЕДЛОЖЕНИЕ 3.1. (i) $W$ переставляет камеры из $\mathscr{M}$ транзитивно.

(ii) Замыкание $\bar{A}$ камеры $А$ является фундаментальной областью для $W$, действующей на $V$.

(iii) Если $x \in V$ принадлежит $\bar{A}$, то стабилизатор $x$ порождается отражениями по отношению $\kappa$ стенкам $A$, содержащим $x$.

Существует множество $I$ и взаимно однозначное соответствие между элементами $I$ и стенками камеры $A: i \mapsto M_{i}(A)$, которое называется канонической индексацией стенок камеры $A$. Тогда $W$ порождается отражениями $w_{i}=w_{i}\left(M_{i}\right), i \in I$, удовлетворяющими только следующим соотношениям:

$$
\left(w_{i} w_{j}\right)^{m_{i, j}}=e, \quad i, j \in I,
$$

где натуральные числа $m_{i, j}=m_{j, i}$ образуют матрицу Кокстера для $W$, по которой граф Kокстера $\Gamma(W)$ для $W$ строится таким образом: вершины соответствуют элементам $i \in I$, причем вершины $i$ и $j$ соединены ребром тогда и только тогда, когда $m_{i, j} \geqslant 3$. Мы используем далее следующее обозначение П. Делиня [58]: $\operatorname{prod}(m ; x, y)$ обозначает произведение $x y x y . . .($ $m$ сомножителей $)$. Обобщенная группа кос $B r(W)$ для $W[14],[58]$ определяется как группа с образуюшими $\left\{s_{i}, i \in I\right\}$ и соотношениями

$$
\operatorname{prod}\left(m_{i, j} ; s_{i}, s_{j}\right)=\operatorname{prod}\left(m_{j, i} ; s_{j}, s_{i}\right)
$$


Представление группы $W$ отсюда получается добавлением соотношений:

$$
s_{i}^{2}=e ; \quad i \in I
$$

В теореме 3.1 мы увидим, что это определение обобшенной группы кос согласуется с нашим общим определением группы кос действия групшы $W(\S 1)$. Мы обозначаем через $\tau_{W}$ канонический гомоморфизм из $\operatorname{Br}(W)$ в $W$. Классические косы из $k$ нитей $B r_{k}$ получены этой конструкцией, когда $W$ есть симметрическая группа на $k+1$ символах. В этом случае $m_{i, i+1}=3$ и $m_{i, j}=2$, если $j \neq i, i+1$.

Теперь пусть $J_{1}, \ldots, J_{s}$ суть множества вершин связных компонент граффа Кокстера $W$. Предположим далее, что $W_{q}$ - подгруппа $W$, порожденная отражениями $w_{i}, i \in J_{q}$. Пусть $V_{q}^{0}$ есть подпространство $V$, состояшее из векторов, инвариантных при действии $W_{q}$. Пусть $V_{q}$ обозначает ортогональное дополнение $V_{q}^{0}$ в $V$, $V_{0}=\bigcap_{1 \leqslant q \leqslant s} V_{q}^{0}$. Тогда мы имеем следуюшие факты [57; гл. V, $\S 3.7$, предложение 5].

ПРЕДЛОЖЕНИЕ 3.2. (i) Группа $W$ есть прямое произведение $W_{q}(1 \leqslant q \leqslant s)$.

(ii) Векторное пространство $V$ есть прямая сумма ортогональных подпространств $V_{1}, \ldots, V_{s}, V_{0}$, инвариантных при действии $W$.

Если $V_{0}=0$, то группа $W$, действующая на $V$, назьвается существенной. В этом случае каждая камера является открытым симплициальным конусом. Зададим некоторое упорядочивание стенок $M_{i}, 1 \leqslant i \leqslant n$, каждой камеры $A$. Произведение отражений $w_{M_{1}} w_{M_{2}} \ldots w_{M_{n}}$ есть преобразование Кокстера, определенное упорядоченной камерой $A$. Все преобразования Кокстера сопряжены в $W$, и, следовательно, все они имеют одинаковый характеристический многочлен и одинаковый (конечный) порядок. Это число называется числом Кокстера группы $W$. Обозначим его через $h$. Тогда характеристический полином преобразования Кокстера может быть записан в виде

$$
f(t)=\prod_{j=1}^{n}\left(t-\exp \left(\frac{2 i \pi m_{j}}{h}\right)\right)
$$

где $m_{1}, m_{2}, \ldots, m_{n}$ - такие числа, что

$$
0 \leqslant m_{1} \leqslant m_{2} \leqslant \cdots \leqslant m_{n}<h .
$$

Числа $m_{1}, m_{2}, \ldots, m_{n}$ назьваются показателямм $W$.

Классификация неприводимых (т.е. со связными графами Кокстера) групп Кокстера хорошо известна (см., например, теорему 1 гл. VI, $\S 4$ книги [57]). Она состоит из трех бесконечных серий: $A, C$ (которую также обозначают через $B$ потому, что в соответствующей классификации простых алгебр Ли две различные серии $B$ и $C$ имеют эту группу в качестве группы Вейля) и $D$, а также исключительных групп: $E_{6}, E_{7}$, $E_{8}, F_{4}, G_{2}, H_{3}, H_{4}$ и $I_{2}(p)$. Например, имеем следующие графы Кокстера для $A_{n}, C_{n}$, $D_{n}$ и $E_{8}$ : 
$\left(A_{n}\right)$

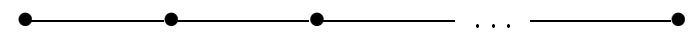

$\left(C_{n}\right)$

4

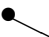

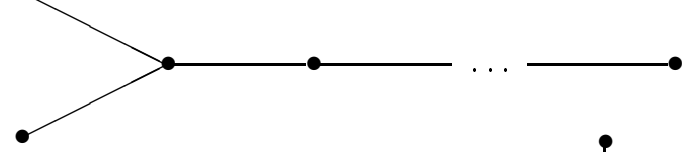

$\left(E_{8}\right)$

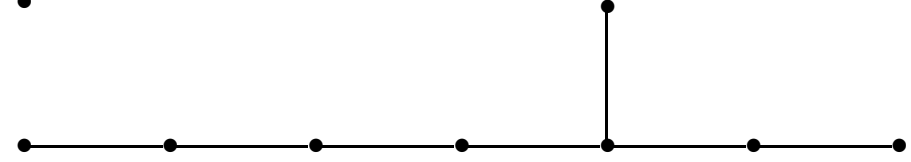

Число вершин в этих диаграммах равно $n$, а число $m$ над ребром означает, что $m_{i, j}=m$ для пары образуюших, соответствующих точкам, соединенным данным ребром. Если $m_{i, j}=3$, то написание числа над ребром опускается.

Рассмотрим теперь комплексификацию $V_{C}$ пространства $V$ и комплексификацию $M_{C}$ для $M \in \mathscr{M}$. Пусть $Y_{W}=V_{C}-\bigcup_{M \in \mathscr{M}} M_{C}$. Из пункта (iii) предложения 3.1 мы заключаем, что $W$ действует свободно на $Y_{W}$. Пусть $X_{W}=Y_{W} / W$, тогда $Y_{W}$ есть накрытие над $X_{W}$, соответствуюшее групе $W$. Пусть $y_{0}$ есть точка в некоторой камере $A_{0}$ и $x_{0}$ - ее образ в $X_{W}$. Мы находимся в ситуации, описанной в $§ 1$, в определении группы кос действия группы $W$. Эта группа кос определялась как фундаментальная группа пространства регулярных орбит действия $W$. В нашем случае $O R B\left(V_{C}, W\right)=X_{W}$. Следовательно, обобщенная группа кос равна $\pi_{1}\left(X_{W}, x_{0}\right)$. Для каждого $j \in I$ пусть $\ell_{j}^{\prime}$ есть гомотопический класс путей в $Y_{W}$, начинающихся в $y_{0}$ и оканчиваюшихся в $w_{j}\left(y_{0}\right)$, которые содержат ломаную линию с последовательными вершинами: $y_{0}, y_{0}+i y_{0}, w_{j}\left(y_{0}\right)+i y_{0}, w_{j}\left(y_{0}\right)$. Образ $\ell_{j}$ класса $\ell_{j}^{\prime}$ в $X_{W}$ является петлей с базисной точкой $x_{0}$.

ТеОрема 3.1. (i) Фундаментальная группа $\pi_{1}\left(X_{W}, x_{0}\right)$ порождена әлементами $\ell_{j}$, удовлетворяющими следующим соотношениям:

$$
\operatorname{prod}\left(m_{j, k} ; \ell_{j}, \ell_{k}\right)=\operatorname{prod}\left(m_{k, j} ; \ell_{k}, \ell_{j}\right) .
$$

(ii) Универсальное накрытие $X_{W}$ стягиваемо, $и$, следовательно, $X_{W}$ есть пространство типа $K(\pi ; 1)$.

Часть (i) этой теоремы была доказана Э. Брискорном [59]. Часть (ii) для групп типов $C_{n}, G_{2}$ и $I_{2}(p)$ была доказана также Э. Брискорном [59] аналогично тому, как Э. Фаделл и Л. Нойвирт [11] доказьвали теоремы $1.1,1.2$ и следствие 1.2. В доказательстве утверждения (ii) для групп типов $D_{n}$ и $F_{4}$ Э. Брискорн использует этот метод с небольшими изменениями. В обшем случае часть (ii) этой теорема была доказана П. Делинем [58]. 
Если $W$ есть прямое произведение $W^{\prime}$ и $W^{\prime \prime}$, то группа $B r(W)$ есть прямое произведение $B r\left(W^{\prime}\right)$ и $B r\left(W^{\prime \prime}\right)$. Следовательно, если группа $W$ такая же, как в предложении 3.2, то $\operatorname{Br}(W)=\operatorname{Br}\left(W_{1}\right) \times \cdots \times \operatorname{Br}\left(W_{s}\right)$.

В терминах графов Кокстера спаривание $\mu$ из (2.4) для классических групп кос означает, что мы берем вершину, соответствующую $\sigma_{k}$ в графе Кокстера $\Gamma\left(\Sigma_{k+l}\right)$, и вкладываем $B r_{k} \times B r_{l}$ в $B r_{k+l}$ в соответствии с вложением $\Gamma\left(\Sigma_{k} \times \Sigma_{l}\right)=\Gamma\left(\Sigma_{k}\right) \cup$ $\Gamma\left(\Sigma_{l}\right)$ в две компоненты графа $\Gamma\left(\Sigma_{k+l}\right) \backslash \sigma_{k}$. Таким же образом мы интерпретируем различные вложения произведений конечных групп Кокстера в группу с большим индексом. Это верно также для соответствующих обобщенных групп кос. Мы удаляем вершину в связном графе Кокстера и получаем граф, у которого число компонент связности меньше или равно 3. Эти компоненты соответствуют неприводимым группам Кокстера или группам кос, прямое произведение которых является областью определения отображения. Например, имеем очевидные спаривания:

$$
\begin{aligned}
& \mu(C, A): \operatorname{Br}\left(C_{k}\right) \times \operatorname{Br}\left(A_{l}\right) \rightarrow B r\left(C_{k+l+1}\right), \\
& \mu(D, A): \operatorname{Br}\left(D_{k}\right) \times B r\left(A_{l}\right) \rightarrow B r\left(D_{k+l+1}\right) \text { для всех } k \text { и } l,
\end{aligned}
$$

или спаривание

$$
\mu\left(A_{3}, A_{4} ; E_{8}\right): B r\left(A_{3}\right) \times B r\left(A_{4}\right) \rightarrow B r\left(E_{8}\right),
$$

которое соответствует четвертой горизонтальной вершине графа Кокстера $E_{8}$.

Вложения групा (не произведений) могут быть также выражены на этом языке. Например, имеем вложение

$$
\alpha_{C}: \operatorname{Br}\left(A_{l-1}\right) \rightarrow B r\left(C_{l}\right)
$$

и два различных вложения:

$$
\alpha_{D}: \operatorname{Br}\left(A_{l-1}\right) \rightarrow \operatorname{Br}\left(D_{l}\right)
$$

в соответствии с двумя различньми вершинами на одном конце графа Кокстера для $D_{l}$.

Теорема 3.1 дает копредставление группы $\operatorname{Br}\left(C_{k}\right)$ образуюшими $\left\{s_{1}, \ldots, s_{k}\right\}$, удовлетворяюшими соотношению

$$
s_{1} s_{2} s_{1} s_{2}=s_{2} s_{1} s_{2} s_{1}
$$

а также соотношениям группы кос (1.2) для образуюших $s_{2}, \ldots, s_{k}$. Пусть $B r_{1, n+1}$ есть подгруппа группы кос $B r_{n+1}$, состояшая из всех элементов $B r_{n+1}$, с тем свойством, что перестановки, им соответствующие, оставляют единицу инвариантной. Это означает, что конец первой нити находится снова на первом месте. В.-Л. Чжоу [60] нашел копредставление этой группы с образующими

$$
\sigma_{2}, \ldots, \sigma_{n}, a_{2}, \ldots, a_{n+1},
$$

где $\sigma_{j}$ есть стандартная образующая группы кос $B r_{n+1}$, а элементы $a_{i}$ заданы равенством $a_{i}=\sigma_{1}^{-1} \ldots \sigma_{i-2}^{-1} \sigma_{i-1}^{2} \sigma_{i-2} \ldots \sigma_{1}, 2 \leqslant i \leqslant n+1$. Элементы $\sigma_{2}, \ldots, \sigma_{n}$ порождают подгруппу в $B r_{1, n+1}$, изоморфную $B r_{n}$, а элементы $a_{2}, \ldots, a_{n+1}$ порождают нормальную свободную подгрупу $F_{n}$. Следуюшее соотношение вьполнено в $B r_{1, n+1}$ :

$$
\sigma_{2} a_{2} \sigma_{2} a_{2}=a_{2} \sigma_{2} a_{2} \sigma_{2} \text {. }
$$

Определим гомоморфизм $\phi: B r\left(C_{n}\right) \rightarrow B r_{1, n+1}$ по формулам:

$$
\begin{aligned}
& \phi\left(w_{1}\right)=a_{2}, \\
& \phi\left(w_{i}\right)=\sigma_{i}, \quad i=2, \ldots, n,
\end{aligned}
$$

и получим следующее утверждение. 
ПРЕДЛОЖЕНИЕ 3.3. Отображсение ф определяет изоморфизм

$$
\phi: B r\left(C_{n}\right) \cong B r_{1, n+1} \text {. }
$$

Это утверждение очевидно с геометрической точки зрения. Пространство $X_{C_{n}}$ можно интерпретировать как пространство $n$ различных пар точек из $\mathbb{R}^{2} \backslash 0$, симметричных относительно нуля [34], [35]. Это то же самое, что просто пространство $n$ различных точек в $\mathbb{R}^{2} \backslash 0$. Группа $B r_{n+1}$ интерпретируется как фундаментальная группа пространства $X_{A_{n}}$ наборов $n+1$ различных точек из $\mathbb{R}^{2}$. Если зафиксировать одну точку (скажем, 0 ), то это будет $X_{C_{n}}$. Для фундаментальной группы $X_{A_{n}}$ это означает, что первая нить должна иметь те же самые начало и конец (равные нулю). Наши рассмотрения означают также, что группа кос $\operatorname{Br}\left(C_{n}\right) \cong B r_{1, n+1}$ может быть интерпретирована как группа кос в полнотории, которая определяется в следующем параграфе.

Пусть $\beta$ есть гомоморфизм из $\operatorname{Br}\left(C_{n}\right)$ в $B r_{n}$, определенный по формулам:

$$
\begin{aligned}
& \beta\left(s_{1}\right)=e, \\
& \beta\left(s_{i}\right)=\sigma_{i-1} \text { для } i>1 .
\end{aligned}
$$

Тогда $\beta \alpha_{C}=1_{B r_{n}}$ и $B r\left(C_{n}\right)$ изоморфна полупрямому произведению $F_{n}$ и $B r_{n}$ с действием $B r_{n}$ на $F_{n}$, описанным в $\S 2$. Известно, что группа $C_{k}$ изоморфна сплетению симметрической групшы $\Sigma_{k}=A_{k-1} \mathrm{c} \mathbb{Z} / 2: C_{k} \cong \Sigma_{k} \int \mathbb{Z} / 2$. Спаривание

$$
m_{C}: C_{k} \times C_{l} \rightarrow C_{k+l}
$$

может быть определено, используя спаривание для симметрических групп

$$
\Sigma_{k} \times \Sigma_{l} \rightarrow \Sigma_{k+l}
$$

и структуру сплетения.

Пусть $s_{1}^{\prime}, \ldots, s_{k}^{\prime}$ суть образующие $\operatorname{Br}\left(C_{k}\right), s_{1}^{\prime \prime}, \ldots, s_{l}^{\prime \prime}$ - образующие $\operatorname{Br}\left(C_{l}\right)$. Тогда мы можем определить спаривание

$$
\mu(C, C): B r\left(C_{k}\right) \times B r\left(C_{l}\right) \rightarrow B r\left(C_{k+l}\right)
$$

по формулам:

$$
\begin{aligned}
& \mu(C, C)\left(s_{i}^{\prime}, e\right)=s_{i}, \quad 1 \leqslant i \leqslant k \\
& \mu(C, C)\left(e, s_{1}^{\prime \prime}\right)=s_{k+1} \ldots s_{2} s_{1} s_{2} \ldots s_{k+1}, \\
& \mu(C, C)\left(e, s_{j}^{\prime \prime}\right)=s_{k+j}, \quad 1 \leqslant j \leqslant l .
\end{aligned}
$$

Соотношение (3.1) показьвает, что образы $s_{1}^{\prime}$ и $s_{1}^{\prime \prime}$ коммутируют в $B r\left(C_{k+l}\right)$. Коммутирование образов $s_{i}^{\prime}, i \geqslant 2$, и $s_{1}^{\prime \prime}$ следует из соотношений группы кос. Следовательно, 
$\mu(C, C)$ есть гомоморфизм. Спаривание $\mu(C, C)$ было впервые определено в [61]. Легко проверить, что $\mu(C, C)$ ассоциативно, т.е. что следуюшая диаграмма коммутативна:

$$
\begin{array}{rrr}
\operatorname{Br}\left(C_{k}\right) \times \operatorname{Br}\left(C_{l}\right) \times \operatorname{Br}\left(C_{q}\right) \stackrel{\mu_{i} \times 1}{\longrightarrow} & \operatorname{Br}\left(C_{k+l}\right) \times \operatorname{Br}\left(C_{q}\right) \\
\lfloor 1 \times \mu(C, C) & & \downarrow \mu(C, C) \\
\operatorname{Br}\left(C_{k}\right) \times \operatorname{Br}\left(C_{l+q}\right) & \stackrel{\mu_{i}}{\longrightarrow} & \operatorname{Br}\left(C_{k+l+q}\right)
\end{array}
$$

Это спаривание согласовано со спариванием для групп Кокстера

$$
m_{C}: C_{k} \times C_{l} \rightarrow C_{k+l},
$$

так, что диаграмма

$$
\begin{array}{rrr}
\operatorname{Br}\left(C_{k}\right) \times \operatorname{Br}\left(C_{l}\right) \stackrel{\tau_{C} \times \tau_{C}}{\longrightarrow} C_{k} \times C_{l} \\
\downarrow \mu(C, C) & & \text { m }_{C} \\
\operatorname{Br}\left(C_{k+l}\right) & \stackrel{\tau_{C}}{\longrightarrow} & C_{k+l}
\end{array}
$$

коммутативна. Спаривание $\mu(C, C)$ также согласовано со спариванием

$$
\operatorname{Br}\left(C_{k}\right) \times B r_{l} \rightarrow B r\left(C_{k+l}\right)
$$

через каноническое вложение $B r_{l} \rightarrow B r\left(C_{l}\right)$. Легко проверить коммутативность диаграммы для гомоморфизма $\alpha_{C}$ :

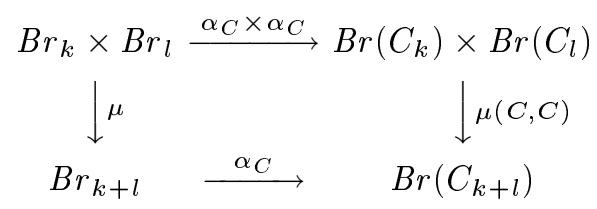

Однако, нет аналогичной коммутативности для $\beta: B r\left(C_{k}\right) \rightarrow B r_{k}$ и $\mu(C, C)$. Действительно, пусть $k=2$. Тогда $\mu(\beta, \beta)\left(e, s_{1}^{\prime \prime}\right)=\mu(e, e)=e$ и $\beta \mu(C, C)\left(e, s_{1}^{\prime \prime}\right)=$ $\beta\left(s_{3} s_{2} s_{1} s_{2} s_{3}\right)=\sigma_{2} \sigma_{1}^{2} \sigma_{2} \neq e$. Следовательно, гомоморфизм $\beta$ не согласован со спариваниями $\mu$ и $\mu(C, C)$.

На уровне конфигурационных пространств спаривание $\mu(C, C)$ для кос серии $C$ может быть описано следуюшим образом. Отобразим $\mathbb{R}^{2} \backslash 0$ (с $k$ различными точками) диффеоморфно на открытый диск радиуса $k+1 / 2$ без нуля: $\mathscr{D}_{k+1 / 2} \backslash 0$ таким образом, что точки с координатами $(1,0), \ldots,(k, 0)$ отображаются на себя. Отобразим также $\mathbb{R}^{2} \backslash 0$ (с $l$ различными точками) диффеоморфно на $\mathbb{R}^{2} \backslash \mathscr{D}_{k+1 / 2}$ таким образом, что точки с координатами $(1,0), \ldots,(l, 0)$ отображаются в точки $(k+1,0), \ldots,(k+l, 0)$. Это отображение

$$
\mathbb{R}^{2} \backslash 0 \times \mathbb{R}^{2} \backslash 0 \rightarrow \mathbb{R}^{2} \backslash 0
$$

является частным случаем отображения конфигурационных пространств:

$$
B(X, k) \times B(X, l) \rightarrow B(X, k+l),
$$


где пространство $X$ может быть представлено в виде $X=Y \times \mathbb{R}$ для некоторого другого пространства $Y$. Отображение (3.2) порождает спаривание фундаментальных групп конфигурационных пространств, которое совпадает с $\mu(C, C)$. Рассматривая обобщенные групшы кос типа $C$ как подгруппы обычных групп кос, мы можем описать спаривание $\mu(C, C)$ через вложение $k+1$ нитей первой группы вместо нулевой нити второй группы.

Рассмотрим теперь прямые пределы конечных групп Кокстера. Обозначим через $\mathscr{W}$ категорию, объектами которой являются конечные группы Кокстера, а морфизмами - вложения $W^{\prime} \mapsto W$, соответствуюшие вложениям графов Кокстера $\Gamma^{\prime} \mapsto \Gamma$. Мы назьваем иепью подкатегорию $\mathscr{E}$ категории $\mathscr{W}$, являюшуюся вполне упорядоченньм множеством и такую, что общее число компонент связности графов Кокстера элементов из $\mathscr{E}$ ограничено некоторым натуральным числом $N_{\mathscr{E}}$ (для подгрупшы $W^{\prime}$ групшы $W$ мы рассматриваем $\Gamma^{\prime}$ как подграф $\Gamma$ ). Мы называем предельной әруппой Коксте$p a W_{\infty}$ такую бесконечную группу, что существует цепь $\mathscr{E}$, для которой $W_{\infty}$ равна прямому пределу $\mathscr{E}$. Если $\mathscr{E}$ есть цепь групп из одной из серий $A, C$ или $D$ с каноническими вложениями в качестве морфизмов, то $A_{\infty}, C_{\infty}$ и $D_{\infty}$ суть соответствуюшие предельные группы Кокстера.

ПРЕДЛОЖЕНИЕ 3.4. Предельная группа Кокстера $W_{\infty}$ изоморфна прямому произведению конечного числа (большего или равного одному) групп $A_{\infty}, C_{\infty}$ или $D_{\infty}$ и произвольному конечному числу конечных групп Кокстера.

Доказательство следует из того факта, что $W_{\infty}$ должна быть бесконечной и ее граф Кокстера должен иметь конечное число компонент.

Некоторые спаривания, описанные вьше, порождают спаривания предельных групп Кокстера и соответствующих групп кос, например:

$$
\begin{aligned}
& \mu(C, A): B r\left(C_{\infty}\right) \times B r\left(A_{\infty}\right) \rightarrow B r\left(C_{\infty}\right), \\
& \mu(D, A): B r\left(D_{\infty}\right) \times B r\left(A_{\infty}\right) \rightarrow B r\left(D_{\infty}\right) .
\end{aligned}
$$

Для произвольной предельной группы Кокстера $W_{\infty}$ может быть несколько различных спариваний с $B r\left(A_{\infty}\right)=B r_{\infty}$, в зависимости от копии одной из предельных групп $A_{\infty}, C_{\infty}$ или $D_{\infty}$, для которой такое спаривание рассматривается

$$
\mu(W, A): B r\left(W_{\infty}\right) \times B r\left(A_{\infty}\right) \rightarrow B r\left(W_{\infty}\right) .
$$

\section{$\S$ 4. Группы кос в телах с ручками}

Подгруппа $B r_{1, n+1}$ групшы кос $B r_{n+1}$, состоящая из кос с прямой первой нитью, рассматривавшаяся в предыдушем параграфе, интерпретировалась также как группа кос в полнотории. Здесь мы изучаем косы в телах с ручками произвольного рода $g$.

Пусть $H_{g}$ есть тело с ручками рода $g$. Группа кос $B r_{n}^{g}$ из $n$ нитей в $H_{g}$ рассматривалась А. Б. Сосинским [15]. В $\S 1$ через $Q_{g}$ было обозначено подмножество многообразия $M$, состоящее из $g$ различных точек. Пусть $M$ есть комплексная плоскость $\mathbb{C}$, $Q_{g}=\left\{z_{1}^{0}, \ldots, z_{g}^{0}\right\}$, скажем, $z_{i}^{0}=i$. Внутренность тела $H_{g}$ имеет интерпретацию как прямое произведение комплексной плоскости $\mathbb{C}$ без $g$ точек $\mathbb{C} \backslash Q_{g}$ на открытый интервал, например, $(-1,2)$ :

$$
\dot{H}_{g}=\left(\mathbb{C} \backslash Q_{g}\right) \times(-1,2) .
$$


Косы из $B r_{n}^{g}$ можно рассматривать лежашими между плоскостями с координатами $z=0$ и $z=1$ и соединяюшими точки $((g+1,0), \ldots,(g+n, 0))$. Так что $B r_{n}^{g}$ можно считать подгруппой классической групшы кос из $g+n$ нитей такой, что косы из $B r_{n}^{g}$ оставляют первые $g$ нитей незаплетенными. В этом параграфе мы обозначаем через $\bar{\sigma}_{j}$ стандартные образуюшие группы кос из $g+n$ нитей. Пусть $\tau_{k}, k=1,2, \ldots, g$, будут следуюшими косами:

$$
\tau_{k}=\bar{\sigma}_{g} \bar{\sigma}_{g-1} \ldots \bar{\sigma}_{k+1} \bar{\sigma}_{k}^{2} \bar{\sigma}_{k+1}^{-1} \ldots \bar{\sigma}_{g-1}^{-1} \bar{\sigma}_{g}^{-1} .
$$

Такая коса изображена на рис. 4.1.

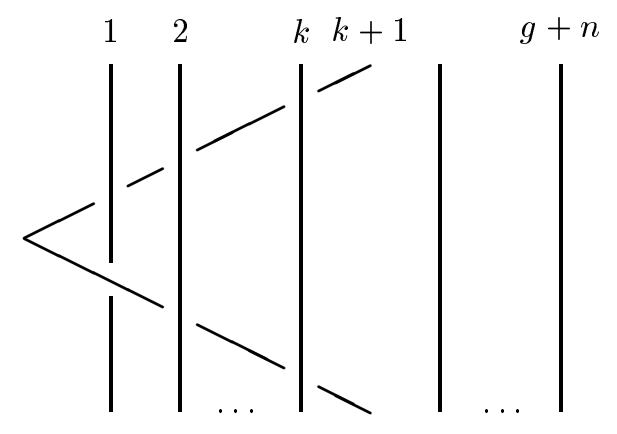

Рис. 4.1

Элементы $\tau_{k}, k=1,2, \ldots, g$, порождают свободную подгруппу $F_{g}$ в группе кос $B r_{g+n}[6],[20],[60]$. Например, из нормальной формы для элементов группы кос (теорема 2.1) следует, что вместе со стандартными образуюшими $\bar{\sigma}_{g+1}, \ldots, \bar{\sigma}_{g+n-1}$ элементы $\tau_{k}, k=1,2, \ldots, g$, порождают группу $B r_{n}^{g}$. Таким образом, группа кос в теле с ручками $B r_{n}^{g}$ может рассматриваться как подгруппа $B r_{g+n}$, порожденная двумя подгруппами: $F_{g}$ и $B r_{n}$. Обозначим через $\sigma_{1}, \ldots, \sigma_{n-1}$ стандартные образуюшие $B r_{n}$, рассматриваемые как элементы $B r_{n}^{g}, \sigma_{i}=\bar{\sigma}_{g+i}, i=1, \ldots, n-1$. Следовательно, они должны удовлетворять соотношениям групшы кос в $B r_{n}^{g}$. Следуюший список соотношений между образуюшими $\tau_{k}$ и $\sigma_{i}$ был получен в [15]:

$$
\left\{\begin{array}{l}
\sigma_{i} \sigma_{j}=\sigma_{j} \sigma_{i}, \text { если }|i-j|>1, \\
\sigma_{i} \sigma_{i+1} \sigma_{i}=\sigma_{i+1} \sigma_{i} \sigma_{i+1} \\
\tau_{k} \sigma_{i}=\sigma_{i} \tau_{k}, \text { если } k \geqslant 1, i \geqslant 2, \\
\tau_{k} \sigma_{1} \tau_{k} \sigma_{1}=\sigma_{1} \tau_{k} \sigma_{1} \tau_{k}, \quad k=1,2, \ldots, g \\
\tau_{k} \sigma_{1}^{-1} \tau_{k+l} \sigma_{1}=\sigma_{1}^{-1} \tau_{k+l} \sigma_{1} \tau_{k}, k=1,2, \ldots, g-1 ; \quad l=1,2, \ldots, g-k .
\end{array}\right.
$$

Соотношения четвертого типа в (4.1) уже появлялись в предыдущем параграфе как формула (3.1) для подгруппы $B r_{1, n}$. Соотношения пятого типа в (4.1) описьвают взаимодействие образующих свободной подгруппы с их ближайшим соседом $\sigma_{1}$. Геометрически это видно на рис. 4.2 . 

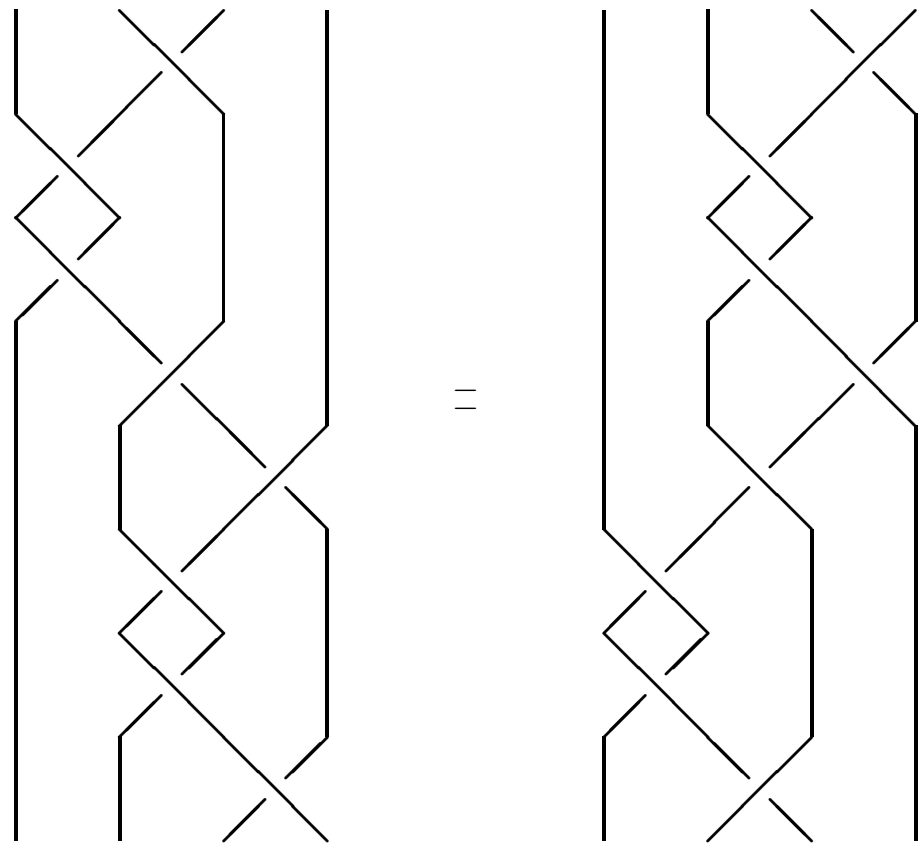

Рис. 4.2

Из теоремы 1.2 следует, что $B\left(\mathbb{C} \backslash Q_{g}, n\right)$ и $F\left(\mathbb{C} \backslash Q_{g}, n\right)$ являются $K(\pi, 1)$-пространствами, $\pi_{1} B\left(\mathbb{C} \backslash Q_{g}\right)=B r_{n}^{g}$, следовательно, $B\left(\mathbb{C} \backslash Q_{g}\right)$ можно рассматривать как классифицируюшее пространство для $B r_{n}^{g}$. Пространство $F\left(\mathbb{C} \backslash Q_{g}, n\right)$ можно интерпретировать как дополнение к конфигурации гиперплоскостей в $\mathbb{C}^{g+n}$, заданной формулами:

$$
\begin{aligned}
& H_{j, k}: z_{j}-z_{k}=0 \text { для всех } j, k \\
& H_{j}^{i}: z_{j}=z_{i}^{0} \text { для } i=1, \ldots, g ; j=1, \ldots, n .
\end{aligned}
$$

В. В. Шехтман и А.Н. Варченко изучали такие конфигурации в [16], где они были названы “дискриминантными".

Имеется аналог теоремы А. А. Маркова (теорема 2.1) для группы $B r_{n}^{g}$.

ПРЕДЛОЖЕНИЕ 4.1. (i) Каждый элемент $\mathrm{Br}_{n}^{g}$ может бъть единственнъцм образом записан в виде

$$
f_{g+1}\left(s_{1, g+1}, \ldots, s_{g, g+1}\right) \ldots f_{g+n}\left(s_{1, g+n}, \ldots, s_{g+n-1, g+n}\right) \sigma_{i_{g+n}, g+n} \ldots \sigma_{i_{g+1}, g+1}
$$

т.е. в представлении (2.7) имеем $f_{j}=1$ и $i_{j}=j$ для $2 \leqslant j \leqslant g$.

(ii) Фактор-группа $P_{m}^{k} / P_{m}^{k-1}$ есть свободная группа от свободных образующих $s_{i, k+1}$.

Доказательство следует из теоремы 2.1. 
Обозначим через $B_{n}^{g}$ группу, заданную абстрактньми образующими $\tau_{k}, 1 \leqslant k \leqslant g$, и $\sigma_{i}, 1 \leqslant i \leqslant n-1$, а также соотношениями (4.1). Сушествует эпиморфизм

$$
\phi_{n}: B_{n}^{g} \rightarrow B r_{n},
$$

определенньй по формулам:

$$
\begin{aligned}
\phi_{n}\left(\sigma_{i}\right) & =\sigma_{i}, \\
\phi_{n}\left(\tau_{k}\right) & =e .
\end{aligned}
$$

Очевидно, что этот эпиморфизм расщепляется.

Пусть $h_{g}$ есть гомоморфизм из группы $B_{n}^{g}$ в группу кос $B r_{g+n}$, определенный следуюшим образом:

$$
\begin{gathered}
\tau_{k} \mapsto \bar{\sigma}_{g} \bar{\sigma}_{g-1} \ldots \bar{\sigma}_{k+1} \bar{\sigma}_{k}^{2} \bar{\sigma}_{k+1}^{-1} \ldots \bar{\sigma}_{g-1}^{-1} \bar{\sigma}_{g}^{-1} \\
\sigma_{i} \mapsto \bar{\sigma}_{g+i}
\end{gathered}
$$

Зададим элементы $a_{i, j}(1 \leqslant i<j, g+1 \leqslant j)$ и элементы $\alpha_{k, l},(g+1 \leqslant k \leqslant l \leqslant g+n)$ из $B_{n}^{g}$ по формулам

$$
\begin{aligned}
a_{i, j} & =\left\{\begin{array}{l}
\tau_{i}, \text { если } j=g+1, \\
\sigma_{j-g-1} \ldots \sigma_{1} \tau_{i} \sigma_{1}^{-1} \ldots \sigma_{j-g-1}^{-1}, \text { если } 1 \leqslant i \leqslant g, g+1<j \leqslant g+n, \\
\sigma_{j-g-1} \ldots \sigma_{i+1-g} \sigma_{i-g}^{2} \sigma_{i+1-g}^{-1} \ldots \sigma_{j-g-1}^{-1}, \text { если } g+1 \leqslant i<j \leqslant g+n,
\end{array}\right. \\
\alpha_{k, k} & =e, \\
\alpha_{k, l} & =\sigma_{k-g} \ldots \sigma_{l-g-1} .
\end{aligned}
$$

Элементы $a_{i, j}$ определены таким образом, что они удовлетворяют соотношениям Бурау (2.5) и их образами в группе $B r_{g+n}$ являются элементы $s_{i, j}$. Образы $\alpha_{k, l}$ суть элементы $\sigma_{k, l}$. Формула (2.6) есть следствие только соотношений Бурау. Следовательно, такая же формула верна для элементов $a_{i, j}$ :

$$
\left[a_{i, l}, a_{j, k}^{\varepsilon}\right]=f\left(a_{1, l}, \ldots, a_{l-1, l}\right), \quad \varepsilon= \pm 1, \quad k<l .
$$

ЛЕмма 4.1. Следующие формуль коммутирования выполнены в $B_{n}^{g}$ :

$$
\sigma_{q}^{\varepsilon_{1}} a_{k, j}^{\varepsilon_{2}}=\left\{\begin{array}{l}
a_{k, j}^{\varepsilon_{2}} \sigma_{q}^{\varepsilon_{1}}, \quad \text { ecлu } q \neq j-g, \quad q \neq j-g-1, \\
a_{k, j+\varepsilon_{1}}^{\varepsilon_{2}} \sigma_{q}^{\varepsilon_{1}}, \quad \text { ecлu } q=j-g, \\
a_{j-1, j} a_{k, j-1} \sigma_{j-g-1}, \quad \text { ecлu } q=j-g-1,
\end{array}\right.
$$

если левая часть не равна $\sigma_{1}^{-1} a_{k, g+1}^{\varepsilon}$. В противном случае имеем

$$
\begin{aligned}
& \sigma_{1}^{-1} a_{k, g+1}=a_{k, g+1} a_{k, g+2} a_{k, g+1}^{-1} \sigma_{1}^{-1}, \\
& \sigma_{1}^{-1} a_{k, g+1}^{-1}=a_{k, g+1} a_{k, g+2}^{-1} a_{k, g+1}^{-1} \sigma_{1}^{-1} .
\end{aligned}
$$


ДокАЗАтЕльство. Следует непосредственно из соотношений. Рассмотрим, например, в (4.3) случай $\sigma_{q} a_{k, j}$, когда $1 \leqslant q<j-g-1$ и $k \leqslant g$. Имеем

$$
\begin{aligned}
\sigma_{q} a_{k, j} & =\sigma_{q} \sigma_{j-g-1} \ldots \sigma_{1} \tau_{k} \sigma_{1}^{-1} \ldots \sigma_{j-g-1}^{-1} \\
& =\sigma_{j-g-1} \sigma_{q} \sigma_{q+1} \sigma_{q} \ldots \sigma_{1} \tau_{k} \sigma_{1}^{-1} \ldots \sigma_{j-g-1}^{-1} \\
& =\sigma_{j-g-1} \sigma_{q+1} \sigma_{q} \sigma_{q+1} \ldots \sigma_{1} \tau_{k} \sigma_{1}^{-1} \ldots \sigma_{j-g-1}^{-1} \\
& =\sigma_{j-g-1} \sigma_{q+1} \sigma_{q} \ldots \sigma_{1} \tau_{k} \sigma_{1}^{-1} \ldots \sigma_{q+1} \sigma_{q}^{-1} \sigma_{q+1}^{-1} \ldots \sigma_{j-g-1}^{-1} \\
& =\sigma_{j-g-1} \sigma_{q+1} \sigma_{q} \ldots \sigma_{1} \tau_{k} \sigma_{1}^{-1} \ldots \sigma_{q}^{-1} \sigma_{q+1}^{-1} \sigma_{q} \ldots \sigma_{j-g-1}^{-1} \\
& =\sigma_{j-g-1} \sigma_{q+1} \sigma_{q} \ldots \sigma_{1} \tau_{k} \sigma_{1}^{-1} \ldots \sigma_{q}^{-1} \sigma_{q+1}^{-1} \sigma_{q} \ldots \sigma_{j-g-1}^{-1} \sigma_{q}
\end{aligned}
$$

Формулы (4.4) и (4.5) суть переписанное четвертое соотношение в (4.1).

Лемма 4.2. Каждый әлемент $B_{n}^{g}$ может быть единственным образом записан в виде

$$
\begin{aligned}
& f_{g+1}\left(a_{1, g+1}, \ldots, a_{g, g+1}\right) \\
& \quad \ldots f_{g+n}\left(a_{1, g+n}, \ldots, a_{g+n-1, g+n}\right) \alpha_{i_{g+n}, g+n} \ldots \alpha_{i_{g+1}, g+1} .
\end{aligned}
$$

ДоКАЗАТЕЛЬСТВо. Применим индукцию по длине $l(z)$ элемента $z \in B_{n}^{g}$ по отношению к базису $\tau_{1}, \ldots, \tau_{g}, \sigma_{1}, \ldots, \sigma_{n}$. Если $l(z)=0$, то $z=1$ и утверждение леммы верно. Пусть $l(z)=l$. Тогда $z=\tau_{k}^{\varepsilon} z_{0}$ или $z=\sigma_{q}^{\varepsilon} z_{0}$, где $l\left(z_{0}\right)<l$. Следовательно, $z_{0}$ представляется в виде (4.6):

$$
\begin{aligned}
z_{0}= & f_{g+1}\left(a_{1, g+1}, \ldots, a_{g, g+1}\right) \\
& \ldots f_{g+n}\left(a_{1, g+n}, \ldots, a_{g+n-1, g+n}\right) \alpha_{i_{g+n}, g+n} \ldots \alpha_{i_{g+1}, g+1} .
\end{aligned}
$$

В первом случае $\tau_{k}=a_{k, g+1}$. Значит,

$$
\begin{aligned}
z=\tau_{k}^{\varepsilon} z_{0}= & a_{k, g+1}^{\varepsilon} f_{g+1}\left(a_{1, g+1}, \ldots, a_{g, g+1}\right) \\
& \ldots f_{g+n}\left(a_{1, g+n}, \ldots, a_{g+n-1, g+n}\right) \alpha_{i_{g+n}, g+n} \ldots \alpha_{i_{g+1}, g+1} \\
= & f_{g+1}^{\prime}\left(a_{1, g+1}, \ldots, a_{g, g+1}\right) \\
& \ldots f_{g+n}\left(a_{1, g+n}, \ldots, a_{g+n-1, g+n}\right) \alpha_{i_{g+n}, g+n} \ldots \alpha_{i_{g+1}, g+1} .
\end{aligned}
$$

Утверждение леммы выполняется в этом случае. Рассмотрим второй случай. Имеем:

$$
\begin{aligned}
z=\sigma_{q}^{\varepsilon} z_{0}= & \sigma_{q}^{\varepsilon} f_{g+1}\left(a_{1, g+1}, \ldots, a_{g, g+1}\right) \\
& \ldots f_{g+n}\left(a_{1, g+n}, \ldots, a_{g+n-1, g+n}\right) \alpha_{i_{g+n}, g+n} \ldots \alpha_{i_{g+1}, g+1} .
\end{aligned}
$$

Используя лемму 4.1, мы можем записать $z$ как слово $f \omega$, где $f$ есть слово из $a_{i, j}$, а $\omega$ - слово из $\sigma_{1}, \ldots, \sigma_{n}$. Элемент $\omega$ можно рассматривать как элемент группы кос $B r_{n}$. Следовательно, по теореме $2.1 \omega$ можно переписать в виде

$$
f_{2}\left(s_{1,2}\right) \ldots f_{j}\left(s_{1, j}, \ldots, s_{j-1, j}\right) \ldots f_{n}\left(s_{1, n}, \ldots, s_{n-1, n}\right) \sigma_{i_{n}, n} \ldots \sigma_{i_{j}, j} \ldots \sigma_{i_{2}, 2} .
$$

Значит, элемент $z$ переписан теперь в виде $f f^{\prime} \alpha$, где $\alpha$ есть $\alpha_{i_{n}, n} \ldots \alpha_{i_{2}, 2}, f$ и $f^{\prime}$ суть слова из $a_{i, j}$. Используя (4.2), перепишем $f f^{\prime}$ в виде

$$
f_{g+1}\left(a_{1, g+1}, \ldots, a_{g, g+1}\right) \ldots f_{g+n}\left(a_{1, g+n}, \ldots, a_{g+n-1, g+n}\right) .
$$


ТЕОРема 4.1. Гомоморфизм $h_{g}$ из $B_{n}^{g}$ в группу кос $B r_{g+n}$ является изоморфизмом группь $B_{n}^{g}$ на группу $\mathrm{Br}_{n}^{g}$.

ДоКАЗАТЕЛЬСтво. Рассмотрим композицию

$$
\nu^{\prime}: B_{n}^{g} \stackrel{\phi}{\longrightarrow} B r_{n}^{g} \stackrel{\nu}{\longrightarrow} \Sigma_{n}
$$

гомоморфизма $\phi$ с каноническим эпиморфизмом

$$
\nu: B r_{n} \rightarrow \Sigma_{n} .
$$

Если элемент $z \in B_{n}^{g}$ записан в виде (4.6), то его образ в $\Sigma_{n}$ равен следующему произведению транспозиций:

$$
\left(i_{n}, n\right) \ldots\left(i_{2}, 2\right)
$$

Следовательно, ядро $J_{g+n}^{g}$ гомоморфизма $\nu_{n}^{\prime}$ порождено $a_{i, j}, 1 \leqslant i<j \leqslant g+n, g<j$. Таким образом, гомоморфизм $h_{g}$ индуцирует морфизм точных последовательностей:

$$
\begin{aligned}
& e \longrightarrow J_{g+n}^{g} \longrightarrow B_{n}^{g} \longrightarrow \Sigma_{n} \longrightarrow e \\
& \downarrow h_{g} \mid J_{g+n}^{g} \quad \downarrow h_{g} \downarrow \\
& e \longrightarrow P_{g+n} \longrightarrow \mathrm{Br}_{g+n} \longrightarrow \Sigma_{g+n} \longrightarrow e
\end{aligned}
$$

Ограничение на подгруппу $B r_{n}^{g}$ группы $B r_{g+n}$ дает следующий морфизм точных последовательностей:

$$
\begin{aligned}
& e \longrightarrow J_{g+n}^{g} \longrightarrow B_{n}^{g} \longrightarrow \Sigma_{n} \longrightarrow e \\
& \downarrow h_{g} \mid J_{g+n}^{g} \quad \downarrow h_{g} \quad \downarrow \text { Id } \\
& e \longrightarrow P_{g+n}^{g} \longrightarrow B r_{n}^{g} \longrightarrow \Sigma_{n} \longrightarrow e
\end{aligned}
$$

Пусть $J_{g+n}^{k}$ есть подгруппа $J_{g+n}^{g}$, порожденная $a_{i, j}, k<j, g \leqslant k$. Из (4.2) следует, что $J_{g+n}^{k}$ есть нормальная подгруппа $J_{g+n}^{g}$. Гомоморфизм $h_{g}$ отображает эту подгрупшу в $P_{g+n}^{g}$. Таким образом, получаем морфизм точных последовательностей:

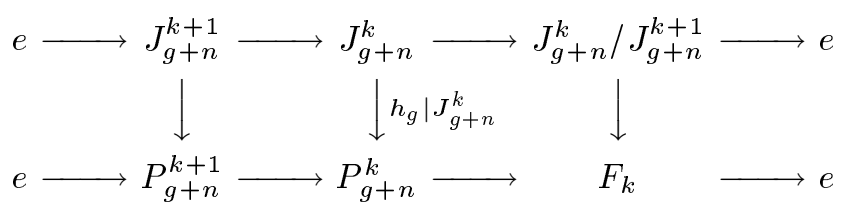

Группа $J_{g+n}^{k} / J_{g+n}^{k+1}$ порождена смежными классами элементов $a_{i, k+1}, 1 \leqslant i \leqslant k$, а их образы в $F_{k}$ суть свободные образующие. Следовательно, правая стрелка в (4.8) есть изоморфизм. Группа $J_{g+n}^{g+n-1}$ порождена $a_{i, g+n}, 1 \leqslant i \leqslant g+n-1$, а их образы в группе $P_{g+n}^{g+n-1}$ - также свободные образующие. Следовательно, имеется изоморфизм

$$
h_{g} \mid J_{g+n}^{g+n-1}: J_{g+n}^{g+n-1} \cong P_{g+n}^{g+n-1} .
$$

По индукции из этого факта и из диаграммы (4.8) получаем, что

$$
h_{g} \mid J_{g+n}^{k}: J_{g+n}^{k} \rightarrow P_{g+n}^{k}
$$

есть изоморфизм для всех $k$. Тогда из диаграммы (4.7) следует, что

$$
h_{g}: B_{n}^{g} \rightarrow B r_{n}^{g}
$$

есть изоморфизм. 
СлЕДСТВИЕ 4.1. Для группь кос $\mathrm{Br}_{n}^{g}$ в теле с ручками рода g существует нормальный ряд

$$
\{e\}=J_{g+n}^{g+n} \subset J_{g+n}^{g+n-1} \subset \cdots \subset J_{g+n}^{g+1} \subset J_{g+n}^{g} \subset B r_{n}^{g}
$$

такой, что

$$
B r_{n}^{g} / J_{g+n}^{g} \cong \Sigma_{n}, J_{g+n}^{r} / J_{g+n}^{r+1} \cong F_{r}, \quad r=g, \ldots, g+n-1
$$

Гомоморфизмы, индуцированные вложением $\mathrm{Br}_{n}^{g} \rightarrow \mathrm{Br}_{g+n}$, отображсают его в соответствующий нормальный ряд группь кос $\mathrm{Br}_{g+n}$ таким образом, что $J_{g+n}^{r} \cong P_{g+n}^{r}$.

ЗАМЕЧАНИЕ. Можно рассмотреть группу кос поверхности (ориентированной или неориентированной) с удаленньми $h$ точками. Группа кос и группа крашеных кос из $m$ нитей замкнутой поверхности были описаны в [62]. Группа крашеных кос имеет образуюшие $A_{i, l}$ и $\rho_{i, k}$, где $A_{i, j}$ суть некоторые образуюшие обычной групшы крашеных кос, а $\rho_{i, k}$ возникают из лент Мёбиуса или ручек поверхности. Факт, аналогичньй теореме 2.1 (i), остается верным [62]. Таким образом, возможно в принципе получить представление группы кос поверхности с удаленными $h$ точками, используя представление группы кос замкнутой поверхности, описанное в [62].

Теорема 4.1 и следствие 4.1 были доказаны в [63].

\section{$\S$ 5. Косы с особенностями}

Пусть $B P_{n}$ есть подгруппа группы Aut $F_{n}$, порожденная двумя множествами автоморфизмов $\xi_{i}$ и $\sigma_{i}$ из (2.1) и (2.2). Она назьвается группой кос-перестановок. Р. Фенн, Р. Риманьи и К. Рурк доказали [23], [24], что $B P_{n}$ задается набором образуюших $\left\{\xi_{i}, \sigma_{i}, i=1,2, \ldots, n-1\right\}$ и соотношениями:

$$
\begin{aligned}
& \left\{\begin{array}{l}
\xi_{i}^{2}=1, \\
\xi_{i} \xi_{j}=\xi_{j} \xi_{i}, \text { если }|i-j|>1, \\
\xi_{i} \xi_{i+1} \xi_{i}=\xi_{i+1} \xi_{i} \xi_{i+1}
\end{array}\right. \\
& \left\{\begin{array}{l}
\sigma_{i} \sigma_{j}=\sigma_{j} \sigma_{i}, \text { если }|i-j|>1, \\
\sigma_{i} \sigma_{i+1} \sigma_{i}=\sigma_{i+1} \sigma_{i} \sigma_{i+1}
\end{array}\right. \\
& \left\{\begin{array}{l}
\sigma_{i} \xi_{j}=\xi_{j} \sigma_{i}, \text { если }|i-j|>1, \\
\xi_{i} \xi_{i+1} \sigma_{i}=\sigma_{i+1} \xi_{i} \xi_{i+1}, \\
\sigma_{i} \sigma_{i+1} \xi_{i}=\xi_{i+1} \sigma_{i} \sigma_{i+1}
\end{array}\right.
\end{aligned}
$$

Р. Фенн, Р. Риманьи и К. Рурк дали также геометрическую интерпретацию $B P_{n}$ как группы кос со спайками. Сначала они определили диаграмму кос со спайками из $n$ нитей как набор $n$ монотонных дуг, начинающихся из $n$ точек на горизонтальной линии некоторой плоскости (верх диаграммы) и идущих вниз до $n$ точек на другой горизонтальной линии (низ диаграммы). Диаграммы могут иметь пересечения двух 
типов: 1) как обычные косы, что показано на рис. 5.1, или 2) точки спайки, как показано на рис. 5.2 .
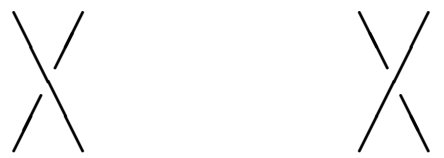

Рис. 5.1

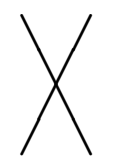

Рис. 5.2

Пример косы со спайками показан на рис. 5.3.

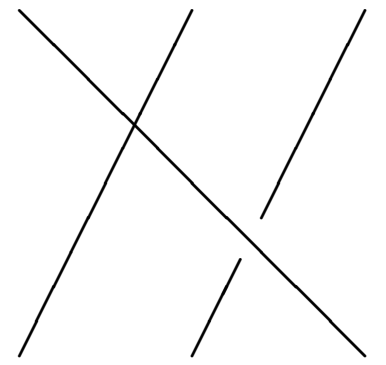

Рис. 5.3

Композиция диаграмм кос со спайками из $n$ нитей определяется расположением одной диаграммы под другой. Диаграмма без пересечений и точек спайки является единичной по отношению к этой композиции. Таким образом, множество диаграмм кос со спайками из $n$ нитей есть полугруппа, обозначаемая через $W D_{n}$.

Р. Фенн, Р. Риманьи и К. Рурк определили следующие типы допустимых преобразований диаграмм кос со спайками. Они изображены на рис. 5.4-5.7.
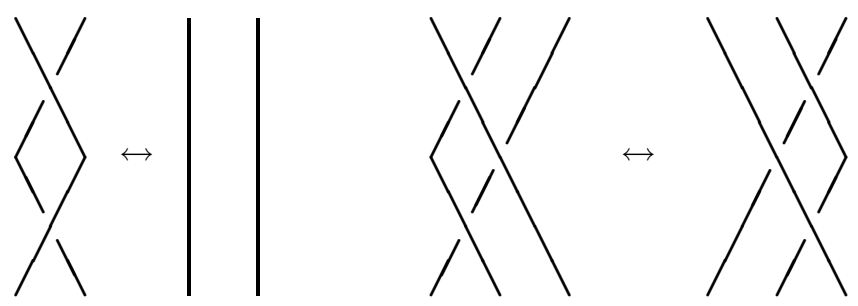

Рис. 5.4 

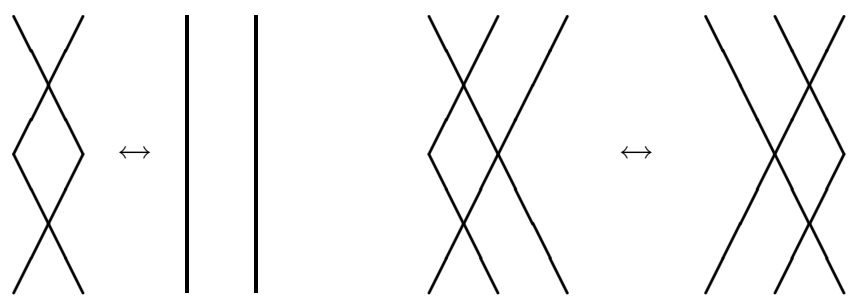

Рис. 5.5
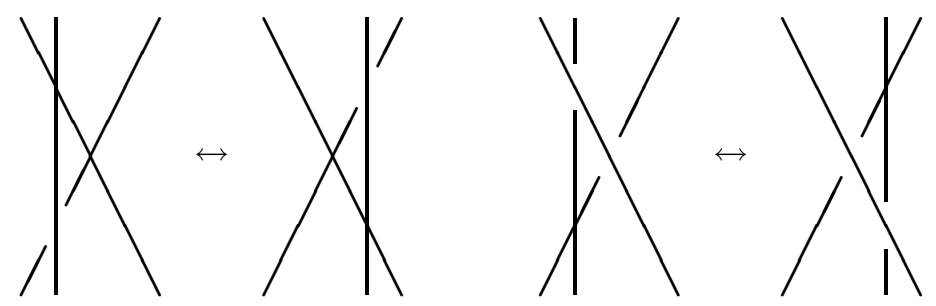

Рис. 5.6

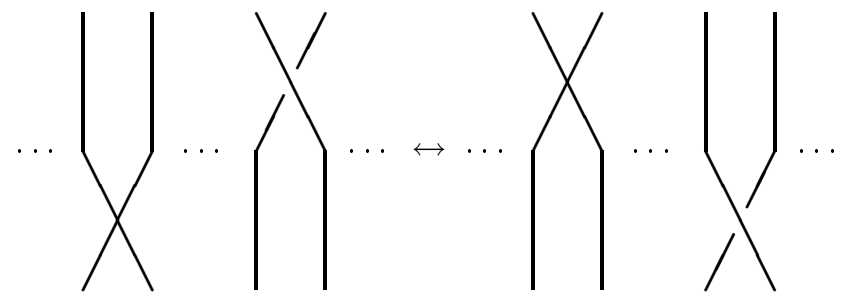

Рис. 5.7

Допустимые преобразования, изображенные на рис. 5.4, суть преобразования Райдемайстера, известные в теории узлов. Первое преобразование на рис. 5.4 соответствует соотношению

$$
\xi_{i}^{2}=1
$$

Преобразование на рис. 5.7 есть геометрическая форма коммутативности из смешанных соотношений.

Коса со спайками определяется как класс эквивалентности диаграмм кос со спайками при допустимых преобразованиях. Р. Фенн, Р. Риманьи и К. Рурк доказали, что косы со спайками образуют группу, и эта группа изоморфна группе кос-перестановок $B P_{n}$. Образуюшая $\sigma_{i}$ соответствует канонической образующей группы кос $B r_{n}$ и изображена на рис. 1.1. Образующие $\xi_{i}$ соответствуют косам со спайками, изображенным на рис. 5.8 . 


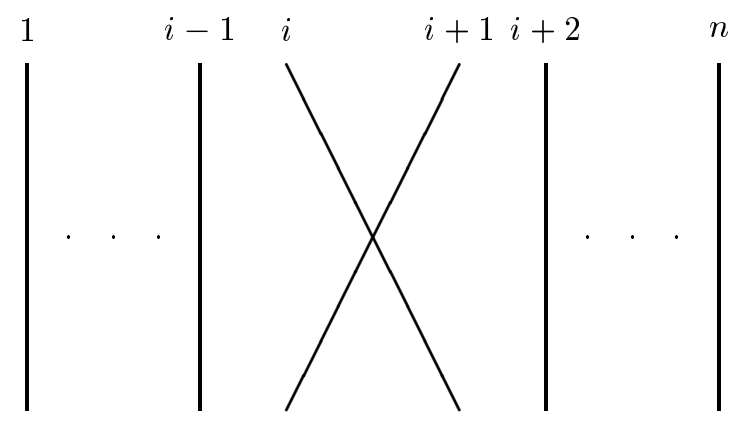

Рис. 5.8

Можно также рассматривать косы со спайками как объекты трехмерного пространства. Мы рассматриваем их вложенными в положительное полупространство (например, по третьей координате) трехмерного евклидова пространства, в то время как точки спайки предполагаются принадлежащими двумерной плоскости с нулевой третьей координатой. Таким образом, нитям не разрешается проходить под точками спайки.

Автоморфизмы группы $F_{n}$, лежащие в $B P_{n}$, могут быть охарактеризованы следующим образом [24]. Пусть $\pi \in \Sigma_{n}$ есть перестановка, а $w_{i}, i=1,2 \ldots, n$, суть слова в $F_{n}$. Тогда отображение

$$
x_{i} \mapsto w_{i}^{-1} x_{\pi(i)} w_{i}
$$

определяет инъективный эндоморфизм $F_{n}$. Если он также сюръективен, то он называется автоморфизмом типа "перестановка-сопряжение". Автоморфизмы этого типа образуют подгруппу группы Aut $F_{n}$, которая является в точности $B P_{n}$. Из этой интерпретации мы видим, что спаривание

$$
\mu(n, m): B P_{n} \times B P_{m} \rightarrow B P_{n+m}
$$

и вложения

$$
B P_{n} \rightarrow B P_{n+k}
$$

корректно определены и удовлетворяют условиям, которые предъявлялись для системы групп $G_{m} \leqslant$ Aut $F_{n}$ в $\S 2$. Система групп $B P_{n}$ согласована со спариванием (2.13) для групп автоморфизмов свободных групп. Действительно, пусть $\alpha \in B P_{k}$ и $\beta \in B P_{l}$ заданы по формулам

$$
\begin{aligned}
& \alpha: x_{j} \mapsto w_{j}^{-1} x_{\pi(j)} w_{j} \\
& \beta: y_{i} \mapsto v_{i}^{-1} y_{\sigma(i)} v_{i}
\end{aligned}
$$

Обозначим через $z_{p, j}$ следующее выражение из букв $a_{i, j}(\S 2)$ :

$$
w_{j}^{-1}\left(a_{p, 1}, \ldots, a_{p, k}\right) a_{p, \pi(j)} w_{j}\left(a_{p, 1}, \ldots, a_{p, k}\right) .
$$

Тогда $\alpha \circ \beta \in$ Aut $F_{k l}$ выражается следующим образом:

$$
\begin{aligned}
a_{i, j} \mapsto v_{i}^{-1}\left(z_{1, j}, \ldots, z_{l, j}\right) w_{j}^{-1} & \left(a_{\sigma(i), 1}, \ldots, a_{\sigma(i), k}\right) \\
& \times a_{\sigma(i), \pi(j)} w_{j}\left(a_{\sigma(i), 1}, \ldots, a_{\sigma(i), k}\right) v_{i}\left(z_{1, j}, \ldots, z_{l, j}\right) .
\end{aligned}
$$


Следовательно, он принадлежит группе кос-перестановок $B P_{k l}$ и спаривание

$$
B P_{k} \times B P_{l} \rightarrow B P_{k l}
$$

корректно определено.

Рассмотрим систему груп $\overline{B P}_{m}$ с теми же образуюшими, что и для групшы кос-перестановок, и такую, что к соотношениям в $B P_{m}$ мы добавляем еше две серии соотношений:

$$
\begin{aligned}
\xi_{i} \sigma_{i+1} \sigma_{i} & =\sigma_{i+1} \sigma_{i} \xi_{i+1} \\
\xi_{i} \sigma_{i} & =\sigma_{i} \xi_{i} .
\end{aligned}
$$

Назовем эту группу редуцированной группой кос-перестановок. Таким образом, смешанные соотношения для редуцированной группы кос-перестановок имеют следующий вид:

$$
\left\{\begin{array}{l}
\sigma_{i} \xi_{j}=\xi_{j} \sigma_{i}, \text { если }|i-j| \neq 1, \\
\xi_{i} \xi_{i+1} \sigma_{i}=\sigma_{i+1} \xi_{i} \xi_{i+1}, \\
\sigma_{i} \sigma_{i+1} \xi_{i}=\xi_{i+1} \sigma_{i} \sigma_{i+1}, \\
\xi_{i} \sigma_{i+1} \sigma_{i}=\sigma_{i+1} \sigma_{i} \xi_{i+1}
\end{array} \quad \text { (смешанные соотношения для } \overline{B P}_{m}\right. \text { ). }
$$

Моноид Баеса-Бирман $S B_{n}$, или моноид обобщенных кос, или моноид кос c особенностя.ми [21], [22], определяется как моноид с образуюшими $g_{i}, g_{i}^{-1}, a_{i}$, $i=1, \ldots, n-1$, и соотношениями

$$
\begin{aligned}
& g_{i} g_{j}=g_{j} g_{i}, \text { если }|i-j|>1, \\
& a_{i} a_{j}=a_{j} a_{i}, \text { если }|i-j|>1, \\
& a_{i} g_{j}=g_{j} a_{i}, \text { если }|i-j| \neq 1, \\
& g_{i} g_{i+1} g_{i}=g_{i+1} g_{i} g_{i+1}, \\
& g_{i} g_{i+1} a_{i}=a_{i+1} g_{i} g_{i+1}, \\
& g_{i+1} g_{i} a_{i+1}=a_{i} g_{i+1} g_{i}, \\
& g_{i} g_{i}^{-1}=g_{i}^{-1} g_{i}=1 .
\end{aligned}
$$

На рисунках $g_{i}$ соответствует канонической образующей групшы кос (правосторонний переход), а $a_{i}$ представляется пересечением $i$-й и $(i+1)$-й нитей, как показано на рис. 1.1 и 5.8. Более детальную геометрическую интерпретацию моноида Баеса-Бирман можно найти в работе Дж. Бирман [22].

Гомоморфизм $j_{n}$ из групшы кос $B r_{n}$ определяется очевидньм образом:

$$
j_{n}: B r_{n} \rightarrow S B_{n}
$$

Р. Фенн, Э. Кейман и К. Рурк доказали [64], что моноид Баеса-Бирман вкладьвается в групшу, которую они назвали группой кос с особенностями:

$$
S B_{n} \rightarrow S G_{n}
$$

Это означает, что в $S G_{n}$ элементы $a_{i}$ становятся обратимьми и все соотношения из $S B_{n}$ остаются справедливьми. 


\section{§6. Классифицирующие пространства для групп автоморфизмов свободных групп}

Под классифипируюшим пространством $B G$ для дискретной группы $G$ мы понимаем пространство Эйленберга-Маклейна $K(G, 1)$. Г. Сегал определил классифицирующее пространство для категории как геометрическую реализацию ее нерва ([65], см. также [66]). В случае, когда категория совпадает с группой, т.е. имеет только один объект, и каждый морфизм обратим, классифицируюшее пространство для категории совпадает с классифицируюшим пространством групшы.

Пусть $G_{n}$ и $H_{n}$ суть системы групп, описанные в $\S 2$. Пусть Aut $\infty$ обозначает объединение всех групп Aut $F_{n}$ при канонических вложениях. Спаривание $\mu_{m, 1}$ из $\S 2$ определяет вложение группы $G_{m}$ в группу $G_{m+1}$. В случае системы групп $H_{m}$ это дает гомоморфизм $H_{m} \rightarrow H_{m+1}$. Тогда группы $G_{\infty}$ и $H_{\infty}$ определяются как прямые пределы систем $\left\{G_{m}\right\}$ и $\left\{H_{m}\right\}$. Пусть $\amalg_{n \geqslant 0} B G_{n}$ и $\amalg_{n \geqslant 0} B H_{n}$ суть моноиды, состояшие из несвязных сумм классифицируюших пространств групा $G_{n}$ и $H_{n}$. Эти моноиды могут быть отождествлены с классифицируюшими пространствами категорий $\mathscr{G}_{\text {и }} \mathscr{H}[66]$.

ПРЕДЛОЖЕНИЕ 6.1. Классифицирующие пространства категорий $\mathscr{G}$ и $\mathscr{H}$ являются $E_{\infty}$-пространствами, для которых имеются очевидние $E_{\infty}$-отображения

$$
B \Sigma \rightarrow B \mathscr{G} \rightarrow B \mathscr{H} .
$$

Имеет место следующая коммутативная диаграмма групповых пополнений

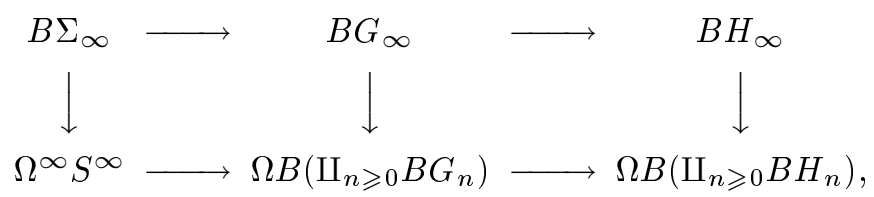

где верхняя строчка индуцирована каноническими групповыми гомоморфизмами

$$
\Sigma_{\infty} \rightarrow G_{\infty} \rightarrow H_{\infty},
$$

а нижняя строчка состоит из бесконечнократных петлевых отображений.

Доказательство основывается на теореме П. Мэя и теореме о групповом пополнении (см., например, [67] или [66; с. $69,90-91])$. Определение $E_{\infty}$-пространств и $E_{\infty}$-отображений дано в дополнении.

Для пространства $X$, имеюшего гомотопический тип $C W$-комплекса, мы используем символ $X^{+}$в том же смысле, в каком и Д.Б. Фукс в [68] использует понятие "квилленизации". Это означает, что $X^{+}$есть гомотопически простой $C W$-комплекс и существует отображение

$$
q: X \rightarrow X^{+},
$$

индуцируюшее изоморфизм в гомологиях. По теореме Дж.Г. К. Уайтхеда $X^{+}$, если существует, то единствен с точностью до гомотопической эквивалентности. Оригинальная плюс-конструкция Д. Квиллена для пространств, у которых коммутант фундаментальной групшы есть совершенная подгруппа, является конструктивным способом построить $X^{+}$в этом случае.

Обозначим через $\Omega_{0} B\left(\amalg_{n \geqslant 0} B G_{n}\right)$ и $\Omega_{0} B\left(\amalg_{n \geqslant 0} B H_{n}\right)$ компоненты связности постоянных отображений в пространствах $\Omega B\left(\amalg_{n \geqslant 0} B G_{n}\right)$ и $\Omega B\left(\amalg_{n \geqslant 0} B H_{n}\right)$. 
СЛЕДСТВИЕ 6.1. При выполнении условий предложения 6.1 имеются изомор$\oint и з м ы$

$$
B G_{\infty}^{+} \cong \Omega_{0} B\left(\amalg_{n \geqslant 0} B G_{n}\right)
$$

$u$

$$
B H_{\infty}^{+} \cong \Omega_{0} B\left(\amalg_{n \geqslant 0} B H_{n}\right),
$$

которые могут быть включень в коммутативную диаграмму, аналогичную (6.1).

ПРЕДЛОЖЕНИЕ 6.2. Существует бесконечнократное пространство петель $X$ такое, что имеет место следующее бесконечнократное петлевое расщепленue:

$$
\Omega B\left(\amalg_{n \geqslant 0} B G_{n}\right) \simeq \Omega^{\infty} S^{\infty} \times X .
$$

ДокАЗАТЕльство. Напомним некоторые результаты $\Phi$. Вальдхаузена [69]. Он изучал пространство

$$
A(*)=\mathbb{Z} \times \underset{n, k}{\underset{\mathrm{lim}}{\longrightarrow}}\left(B \operatorname{Aut}\left(\bigvee^{k} S^{n}\right)\right)^{+}
$$

и доказал, что каноническое отображение

$$
B \Sigma_{\infty}^{+} \rightarrow A(*)
$$

расщепляется, т.е. сушествует отображение

$$
A(*) \rightarrow B \Sigma_{\infty}^{+}
$$

такое, что его композиция с предыдущим отображением есть гомотопическая эквивалентность пространства $B \Sigma_{\infty}^{+}$. Более того, отображения (6.2) и (6.3) являются бесконечнократно петлевыми отображениями между бесконечнократными пространствами петель (см. замечание 5.3 в [69]). А. Хэтчер использовал это расщепление в [70]. Он рассматривал отображение

$$
\operatorname{Aut}\left(\bigvee^{k} S^{0}\right) \rightarrow \operatorname{Aut}\left(\bigvee^{k} S^{1}\right)
$$

индуцированное функтором надстройки, а также композицию

$$
\operatorname{Aut}\left(\bigvee^{k} S^{0}\right) \rightarrow \operatorname{Aut}\left(\bigvee^{k} S^{1}\right) \rightarrow \underset{n, k}{\lim } \operatorname{Aut}\left(\bigvee^{k} S^{n}\right)
$$

Затем он взял композицию (6.4) с отображением $\Phi$. Вальдхаузена (6.3) и получил аналогичное расшепление для $B \mathrm{Aut}_{\infty}^{+}$. Заметим, что аргументы А. Хэтчера остаются справедливыми и для нашей системы подгрупп $G_{n}$. А именно, пусть $X$ есть слой композиции

$$
\Omega B\left(\amalg_{n \geqslant 0} B G_{n}\right) \rightarrow \Omega B\left(\amalg_{n \geqslant 0} B \text { Aut } F_{n}\right) \rightarrow \Omega^{\infty} S^{\infty},
$$

в которой вторым является отображение $\Phi$. Вальдхаузена (6.3). Пространство $X$ является бесконечнократным пространством петель, и бесконечнократно петлевое отображение

$$
\Omega^{\infty} S^{\infty} \rightarrow \Omega B\left(\amalg_{n \geqslant 0} B G_{n}\right)
$$

дает необходимое расшепление. 
ПРЕДЛОЖЕНИЕ 6.3. Если система групп $G_{n}$ согласована со спариванием (2.12)-(2.13), то она определяет мультипликативный, ассочиативный (возможсно, некоммутативный) спектр TG вместе с мультипликативным отображением в сферический спектр

$$
T G \rightarrow S
$$

и это отображсение мультипликативно расщепляется.

ДокАЗАТЕльство. Система групा $G_{n}$ порождает $\Gamma$-категорию в смысле Г. Сегала [71] (см. также дополнение). Эта Г-категория порождает Г-пространство и затем спектр. Если система групп $G_{n}$ из предложения 2.1 согласована со спариванием (2.13), то определено аналогичное спаривание

$$
G_{k} \times G_{l} \rightarrow G_{k l}
$$

Оно определяет умножение соответствующих Г-пространств. Следовательно, спектр, соответствующий бесконечнократному пространству петель $\Omega B\left(\amalg_{n \geqslant 0} B G_{n}\right)$, мультипликативен $[71 ; \S 5]$. Рассмотрим композицию спаривания $(2.13)$ с отображением, порожденным надстройкой

$$
\operatorname{Aut}\left(\bigvee^{k} S^{1}\right) \times \operatorname{Aut}\left(\bigvee^{l} S^{1}\right) \rightarrow \operatorname{Aut}\left(\bigvee^{k l} S^{1}\right) \rightarrow \operatorname{Aut}\left(\bigvee^{k l} S^{2}\right)
$$

Мы получаем спаривание $\operatorname{Aut}\left(\bigvee^{k} S^{1}\right)$ и $\operatorname{Aut}\left(\bigvee^{l} S^{1}\right)$, порожденное смэш-произведением. Это спаривание рассматривалось $\Phi$. Вальдхаузеном [69] при изучении пространства $A(*)$. Канонические отображения

$$
\operatorname{Aut}\left(\bigvee^{k} S^{0}\right) \rightarrow \operatorname{Aut}\left(\bigvee^{k} S^{1}\right) \rightarrow \underset{n, k}{\lim _{n, k}} \operatorname{Aut}\left(\bigvee^{k} S^{n}\right)
$$

согласованы со спариваниями. Ф. Вальдхаузен доказал [69], что отображение (6.3)

$$
A(*) \rightarrow B \Sigma_{\infty}^{+}
$$

согласовано с мультипликативными структурами в $A(*)$ и $B \Sigma_{\infty}^{+}$.

Рассматривая расщепление из предложения 6.2, интересно сравнить гомологии групп $G_{\infty}$ и $\Sigma_{\infty}$. Ответ должен зависеть, конечно, от системы групп $G_{n}$. Например, для самой групшы $\mathrm{Aut}_{\infty}$ эти гомологии в размерностях 1 и 2 совпадают:

$$
H_{i}\left(\mathrm{Aut}_{\infty}, \mathbb{Z}\right)=H_{i}\left(\Sigma_{\infty}, \mathbb{Z}\right) \text { для } i=1,2 .
$$

А. Хэтчер отметил, что в общем случае (для всех $i$ ) неизвестно, справедливо это равенство или нет [70]. Ниже мы будем рассматривать гомологии групшы кос-перестановок. Как мы увидим, этот случай весьма отличен от случая целой группы Aut $_{\infty}$. 


\section{$\S$ 7. Когомологии групп крашеных кос}

Когомологии групп крашеных кос были впервые вычислены В. И. Арнольдом [26], которьй использовал спектральную последовательность Серра. Мы будем рассматривать несколько более обший случай конфигурационного пространства для $\mathbb{R}^{n}[72]$, [73]. Доказательства из работы В. И. Арнольда [26] полностью проходят и в этом случае. Рассмотрим сначала $F\left(\mathbb{R}^{n}, 2\right)$. Отображение

$$
\phi: S^{n-1} \rightarrow F\left(\mathbb{R}^{n}, 2\right),
$$

описьваемое формулой $\phi(x)=(x,-x)$, есть $\Sigma_{2}$-эквивариантная гомотопическая эквивалентность. Обозначим через $A$ образующую $H^{n-1}\left(F\left(\mathbb{R}^{n}, 2\right), \mathbb{Z}\right)$, которая отображается при $\phi^{*}$ в стандартную образующую $H^{n-1}\left(S^{n-1}, \mathbb{Z}\right)$. Для $i$ и $j$ таких, что $1 \leqslant i, j \leqslant m, i \neq j$, определим $\pi_{i, j}: F\left(\mathbb{R}^{n}, m\right) \rightarrow F\left(\mathbb{R}^{n}, 2\right)$ по формуле $\pi_{i, j}\left(p_{1}, \ldots, p_{m}\right)=\left(p_{i}, p_{j}\right)$. Пусть

$$
A_{i, j}=\pi_{i, j}^{*}(A) \in H^{n-1}\left(F\left(\mathbb{R}^{n}, m\right), \mathbb{Z}\right) .
$$

Следовательно, $A_{i, j}=(-1)^{n} A_{j, i}$ и $A_{i, j}^{2}=0$. Для $w \in \Sigma_{m}$ имеется действие $w\left(A_{i, j}\right)=$ $A_{w^{-1}(i), w^{-1}(j)}$, поскольку $\pi_{i, j} w=\pi_{w^{-1}(i), w^{-1}(j)}$. Заметим также, что при ограничении на

$$
F\left(\mathbb{R}^{n} \backslash Q_{k}, m-k\right) \cong \pi^{-1}\left(Q_{k}\right) \subset F\left(\mathbb{R}^{n}, m\right)
$$

классы $A_{i, j}$ с $1 \leqslant i, j \leqslant k$ переходят в нуль, поскольку в этом случае отображение $\pi_{i, j}$ является постоянным на $\pi^{-1}\left(Q_{k}\right)$. Рассматривая спектральную последовательность Серра, получаем следуюший факт.

ТЕОрема 7.1. Группа когомологий $H^{*}\left(F\left(\mathbb{R}^{n} \backslash Q_{k}, m-k\right), \mathbb{Z}\right)$ является свободной абелевой әруппой с образующими

$$
A_{i_{1}, j_{1}} A_{i_{2}, j_{2}} \ldots A_{i_{s}, j_{s}}
$$

где $k<j_{1}<j_{2}<\cdots<j_{s} \leqslant m u i_{r}<j_{r}$ nрu $r=1, \ldots, s$.

Мультипликативная структура и структура $\Sigma_{m}$-алгебры на $H^{*}\left(F\left(\mathbb{R}^{n}, m\right), \mathbb{Z}\right)$ дается следуюшей теоремой, которая доказьвается с использованием $\Sigma_{3}$-действия на $H^{*}\left(F\left(\mathbb{R}^{n}, 3\right), \mathbb{Z}\right)$.

ТЕОРема 7.2. Кольцо когомологий $H^{*}\left(F\left(\mathbb{R}^{n}, m\right), \mathbb{Z}\right)$ мультипликативно порождено әлементами

$$
A_{i, j} \in H^{n-1}\left(F\left(\mathbb{R}^{n}, m\right), \mathbb{Z}\right), \quad 1 \leqslant i<j \leqslant m,
$$

квадраты которых равны нулю, и связанными только соотношениями

$$
A_{i, k} A_{j, k}=A_{i, j} A_{j, k}-A_{i, j} A_{i, k} \quad \text { npu } \quad i<j<k .
$$

Ряд Пуанкаре для $F\left(\mathbb{R}^{n}, m\right)$ есть произведение $\prod_{j=1}^{m-1}\left(1+j t^{n-1}\right)$. 
ЗАмечАниЕ. В случае $\mathbb{R}^{2}=\mathbb{C}$ классы когомологий $A_{j, k}$ могут быть интерпретированы как классы когомологий дифференциальных форм

$$
\omega_{j, k}=\frac{1}{2 \pi i} \frac{d z_{j}-d z_{k}}{z_{j}-z_{k}} .
$$

Э. Брискорн вычислил когомологии крашеных обобщенных групп кос [14], используя идеи В. И. Арнольда для классического случая. Пусть $\mathscr{V}$ есть конечномерное комплексное векторное пространство, а $H_{j} \in \mathscr{V}, j \in I$, есть конечное семейство аффинных гиперплоскостей, заданное линейными формами $l_{j}$. Э. Брискорн доказал следуюший факт.

Теорема 7.3. Классы когомологий, соответствующие голоморфным дифференциальныц формам

$$
\omega_{j}=\frac{1}{2 \pi i} \frac{d l_{j}}{l_{j}},
$$

порождают кольцо когомологий $H^{*}\left(\mathscr{V} \backslash \bigcup_{j \in I} H_{j}, \mathbb{Z}\right)$. Более того, это кольцо изоморфно $\mathbb{Z}$-подалгебре, порожденной формами $\omega_{j}$ в алгебре мероморфных форм на $\mathcal{V}$.

Когомологии крашеных обобшенных групп кос описьваются следуюшим образом.

Tеорема 7.4. (i) Группа когомологий $H^{k}(P(W), \mathbb{Z})$ группы крашеных обобщенных кос $P(W)$ с целыми коэффичиентами является свободной абелевой группой, u ее ранг равен числу әлементов $w \in W$ длины $l(w)=k$, где $l$ есть длина, рассматриваемая по отношению к системе образующих, состоящей из всех отражений $W$.

(ii) Ряд Пуанкаре для $H^{*}(P(W), \mathbb{Z})$ есть произведение $\prod_{j=1}^{n}\left(1+m_{j} t\right)$, где $m_{j}$ суть показатели группь $W$, определенные в $\S 3$.

(iii) Мультипликативная структура $H^{*}(P(W), \mathbb{Z})$ совпадает со структурой алгебры, порожденной 1-формами, описанной в предьдущей теореме.

В. А. Голубева и В.П. Лексин изучали структуру кольца $H^{*}(P(W), \mathbb{Z})$ в терминах квадратичных соотношений, аналогичных (7.1) [74].

\section{§ 8. Гомологии групп кос и пространства петель}

Для изучения когомологий классических групп кос $H^{*}\left(B r_{n}, \mathbb{Z}\right)$ В. И. Арнольд [12] интерпретировал пространство $K\left(B r_{n}, 1\right) \cong B\left(\mathbb{R}^{2}, n\right)$ как пространство комплексных многочленов степени $n$ без кратных корней и со старшим коэффициентом, равным 1 :

$$
P_{n}(t)=t^{n}+z_{1} t^{n-1}+\cdots+z_{n-1} t+z_{n} .
$$

Более точно, рассмотрим "отображение Виета" из $\mathbb{C}^{n}$, которое мы в этом месте обозначаем через $\mathbb{C}_{(\lambda)}^{n}$, в $\mathbb{C}^{n}$, которое мы обозначаем через $\mathbb{C}_{(z)}^{n}$, чтобы различить область определения и область значений:

$$
p: \mathbb{C}_{(\lambda)}^{n} \rightarrow \mathbb{C}_{(z)}^{n}
$$


Оно отображает точку $\left(\lambda_{1}, \ldots, \lambda_{n}\right) \in \mathbb{C}_{(\lambda)}^{n}$ в полином $P_{n}(t)=t^{n}+z_{1} t^{n-1}+\cdots+$ $z_{n-1} t+z_{n}$, который имеет корни $\lambda_{1}, \ldots, \lambda_{n}$ (с учетом кратности). Пространство орбит $\operatorname{Orb}\left(\mathbb{C}^{n}, \Sigma_{n}\right)$ канонического действия симметрической группы $\Sigma_{n}$ на $\mathbb{C}^{n}$ изоморфно $\mathbb{C}_{(z)}^{n}$ при помоши отображения, принимаюшего значения базисных симметрических многочленов от $\lambda_{1}, \ldots, \lambda_{n}$. Стандартный базис состоит из симметрических многочленов:

$$
z_{k}(\lambda)=(-1)^{k} \sum_{i_{1}<\cdots<i_{k}} \lambda_{i_{1}} \ldots \lambda_{i_{k}}, \quad 1 \leqslant k \leqslant n .
$$

Таким образом, $\mathbb{C}_{(z)}^{n} \cong \operatorname{Orb}\left(\mathbb{C}^{n}, \Sigma_{n}\right)$, и $O R B\left(\mathbb{C}^{n}, \Sigma_{n}\right)$ есть пространство регулярных орбит этого действия. Мы увидим сейчас, что отображение $p$ из (8.2) определяет гомеоморфизм

$$
p: \operatorname{ORB}\left(\mathbb{C}^{n}, \Sigma_{n}\right) \rightarrow \mathbb{C}_{(z)}^{n} \backslash \Delta,
$$

где $\Delta$ есть дискриминантная поверхность полинома (8.1), т.е. подпространство $\mathbb{C}^{n}$, где дискриминант $\Delta^{n}(z)$ равен нулю;

$$
\Delta^{n}(z)=\Delta^{n}(p(\lambda))=\prod_{i \neq j}\left(\lambda_{i}-\lambda_{j}\right)=(-1)^{\frac{n(n-1)}{2}} \prod_{i<j}\left(\lambda_{i}-\lambda_{j}\right)^{2} .
$$

Далее В.И. Арнольд рассматривал каноническое вложение пространства $\mathbb{C}_{(z)}^{n} \backslash \Delta$ в сфферy $S^{2 n}$ :

$$
\mathbb{C}_{(z)}^{n} \backslash \Delta \rightarrow S^{2 n}
$$

Дополнение $\mathbb{C}_{(z)}^{n} \backslash \Delta$ в $S^{2 n}$ эквивалентно одноточечной компактификации $\Delta^{*}$ пространства $\Delta$. Двойственность Александера дает

$$
H^{i}\left(B r_{n} ; \mathbb{Z}\right)=H^{i}\left(\mathbb{C}_{(z)}^{n} \backslash \Delta ; \mathbb{Z}\right) \cong H_{2 n-i}\left(S^{2 n}, \Delta^{*} ; \mathbb{Z}\right) \cong \widetilde{H}_{2 n-i+1}\left(\Delta^{*} ; \mathbb{Z}\right),
$$

где волна обозначает приведенные гомологии. В. И. Арнольд доказал следуюшие факты.

Теорема 8.1 (конечности). Когомологии каждой группы кос конечны, кроме

$$
H^{0}\left(B r_{n}, \mathbb{Z}\right) \cong \mathbb{Z}, \quad H^{1}\left(B r_{n}, \mathbb{Z}\right) \cong \mathbb{Z}, \quad n \geqslant 2 .
$$

Также имеем

$$
H^{i}\left(B r_{n}, \mathbb{Z}\right)=0 \quad n p u \quad i \geqslant n .
$$

СлЕДСТвИЕ 8.1. Эйлерова характеристика любой группы кос равна нулю.

ТЕОРема 8.2 (повторения). Все группы когомологий каждой группы кос из нечетного числа нитей такие же, как у группь кос из предидущего четного числа нитей:

$$
H^{i}\left(B r_{2 n+1}, \mathbb{Z}\right)=H^{i}\left(B r_{2 n}, \mathbb{Z}\right) .
$$


ТЕОРема 8.3 (стабилизации). При увеличении п группа когомологий $H^{i}\left(B r_{n}, \mathbb{Z}\right)$ стабилизируется:

$$
H^{i}\left(B r_{n}, \mathbb{Z}\right)=H^{i}\left(B r_{2 i-2}, \mathbb{Z}\right) \quad n p u \quad n \geqslant 2 i-2 .
$$

ЗАмЕчАниЕ. Изоморфизм $H^{1}\left(B r_{n}, \mathbb{Z}\right) \cong \mathbb{Z}$ следует из того факта, что абелеанизация $B r_{n}$ равна $\mathbb{Z}$. Действительно, мы можем определить гомоморфизм из кос в целые числа, беря сумму показателей вхождений образующих $\sigma_{i}$ в выражение любого элемента группы через канонические образующие:

$$
\operatorname{deg}: B r_{n} \rightarrow \mathbb{Z}, \quad \operatorname{deg}(b)=\sum_{j} m_{j}, \quad \text { где } b=\left(\sigma_{i_{1}}\right)^{m_{1}} \ldots\left(\sigma_{i_{k}}\right)^{m_{k}} .
$$

Легко доказать, что ядро deg порождено коммутаторами. Равенство (8.3) следует из того факта, что пространство $C_{(z)}^{n} \backslash \Delta$ есть прямое произведение $\mathbb{C}^{1}$ на многообразие Штейна.

В. И. Арнольд также вычислил групшы $H^{i}\left(B r_{n}, \mathbb{Z}\right)$ при $n \leqslant 11$ и $i \leqslant 9$.

Д.Б. Фукс описал когомологии групп кос по модулю 2 [27]. Обозначим для простоты через $\Gamma_{n}$ конфигурационное пространство $B\left(\mathbb{R}^{2}, n\right)=B(\mathbb{C}, n)=F(\mathbb{C}, n) / \Sigma_{n}$. Пусть $\Gamma_{n}^{*}$ есть одноточечная компактификация $\Gamma_{n}$. Двойственность Пуанкаре дает:

$$
H^{k}\left(\Gamma_{n}, \mathbb{Z} / 2\right) \cong \widetilde{H}_{2 n-k}\left(\Gamma_{n}^{*}, \mathbb{Z} / 2\right) .
$$

Чтобы изучить группу $\widetilde{H}_{j}\left(\Gamma_{n}^{*}, \mathbb{Z} / 2\right)$, Д. Б. Фукс построил некоторое естественное клеточное разбиение пространства $\Gamma_{n}^{*}$. Используя это разбиение, он вычислил группы $H^{i}\left(B r_{n}, \mathbb{Z} / 2\right)$, а также описал мультипликативную структуру кольца $H^{*}\left(B r_{n}, \mathbb{Z} / 2\right)$ и связи с когомологиями $H^{*}\left(B O_{n}, \mathbb{Z} / 2\right)$. Структура алгебры Хопфа когомологий группы кос от бесконечного числа нитей $H^{*}\left(B r_{\infty}, \mathbb{Z} / 2\right)$, возникающая из канонического спаривания (2.4), была также определена [27]. Результаты были сформулированы на языке когомологий. Более удобно их перевести на язык гомологий следуюшим образом.

Теорема 8.4. Гомологии группы кос от бесконечного числа нитей с коэффичиентами в $\mathbb{Z} / 2$ как алгебра Хопфа изоморфны полиномиальной алгебре от бесконечного числа образующих $a_{i}, i=1,2, \ldots ; \operatorname{deg} a_{i}=2^{i}-1$ :

$$
H_{*}\left(B r_{\infty}, \mathbb{Z} / 2\right) \cong \mathbb{Z} / 2\left[a_{1}, a_{2}, \ldots, a_{i}, \ldots\right]
$$

с копроизведением, заданным формулами:

$$
\Delta\left(a_{i}\right)=1 \otimes a_{i}+a_{i} \otimes 1 .
$$

Tеорема 8.5. Каноническое вложсене $\mathrm{Br}_{n} \rightarrow \mathrm{Br}_{\infty}$ индуцирует мономорфизм в гомологиях с коэффициентами в $\mathbb{Z} / 2$. Его образом является подкоалгебра полиномиальной алгебры $\mathbb{Z} / 2\left[a_{1}, a_{2}, \ldots, a_{i}, \ldots\right]$ с $\mathbb{Z} / 2$-базисом, состоящим из мономов

$$
a_{1}^{k_{1}} \ldots a_{l}^{k_{l}} \quad \text { maкux, что } \quad \sum_{i} k_{i} 2^{i} \leqslant n .
$$


Tеорема 8.6. Канонический гомоморфизм $\mathrm{Br}_{n} \rightarrow B O_{n}, 1 \leqslant n \leqslant \infty$, индуиирует мономорфизм (алгебр Хопфа, если $n=\infty)$

$$
H_{*}\left(B r_{n}, \mathbb{Z} / 2\right) \rightarrow H_{*}\left(B O_{n}, \mathbb{Z} / 2\right) .
$$

Независимо от работ В. И. Арнольда и Д. Б. Фукса Ф.Р. Коэн также изучал гомологии групп кос [28], [29], [32]. Он использовал спектральную последовательность накрытия, имеющую начальньй член

$$
E_{2}^{*, *} \cong H^{*}\left(\Sigma_{m} ; H^{*}\left(F\left(\mathbb{R}^{n}, m\right) ; \mathbb{Z} / p\right)\right)
$$

и сходящуюся к $H^{*}\left(B\left(\mathbb{R}^{n}, m\right) ; \mathbb{Z} / p\right)$. Когомологии симметрической группы $\Sigma_{m}$ рассматриваются с нетривиальным действием $\Sigma_{m}$ на группе коэффициентов $H^{*}\left(F\left(\mathbb{R}^{n}, m\right) ; \mathbb{Z} / p\right)$, описанной в теореме 7.2. Ключевым моментом для вычислений является следуюший факт.

ТЕОРема 8.7 (об обнулении). $B$ спектральной последовательности $\left\{E_{r}\right\}$ $E_{2}^{s, t}=0$ nрu $s>0 u 0<t<(n-1)(p-1)$.

$\Phi$. Р. Коэн вычислил гомологии $B\left(\mathbb{R}^{n}, m\right)$ с коэффициентами в $\mathbb{Z} / 2$ (как в теоремах 8.4 и 8.5) и с коэффициентами в $\mathbb{Z} / p, p>2$.

ТЕОрема 8.8. Гомологии группы кос от бесконечного числа нитей с коэффиииентами в $\mathbb{Z} / p, p>2$, как алгебра Хопфа изоморфны тензорному произведению внешней и полиномиальной алгебр:

$$
\begin{gathered}
E\left(a_{1}, \ldots, a_{i}, \ldots\right) \otimes \mathbb{Z} / p\left[b_{1}, \ldots, b_{j}, \ldots\right], \quad i=1,2, \ldots ; \quad j=1,2, \ldots ; \\
\operatorname{deg} a_{i}=2 p^{i-1}-1, \quad \operatorname{deg} b_{j}=2 p^{j}-2,
\end{gathered}
$$

с копроизведением, заданным по формулам:

$$
\Delta\left(a_{i}\right)=1 \otimes a_{i}+a_{i} \otimes 1, \quad \Delta\left(b_{j}\right)=1 \otimes b_{j}+b_{j} \otimes 1 .
$$

TеОРема 8.9. Каноническое вложсение $\mathrm{Br}_{n} \rightarrow B r_{\infty}$ индуиирует мономорфизм в гомологиях с коэффичиентами в $\mathbb{Z} / p, p>2$. Его образом является подкоалгебра тензорного произведения $E\left(a_{1}, \ldots, a_{j}, \ldots\right) \otimes \mathbb{Z} / p\left[b_{1}, \ldots, b_{r}, \ldots\right] c \mathbb{Z} / p$-базисом, состоящим из мономов

$$
a_{1}^{\varepsilon_{1}} \ldots a_{l}^{\varepsilon_{l}} b_{1}^{k_{1}} \ldots b_{s}^{k_{s}}, \quad \text { əде } \quad \varepsilon_{i}=0,1 ; \quad u \quad 2\left(\sum_{i} \varepsilon_{i} p^{i-1}+\sum_{j} k_{j} p^{j}\right) \leqslant n .
$$

$\Phi$. Р. Коэн описал также действие алгебры Стинрода $\mathscr{A}[30]$. Мы выражаем его результаты на языке кодействия $\psi$ алгебры $\mathscr{A}_{*}$, двойственной к алгебре Стинрода. При $p=2 \mathscr{A}_{*}$ как алгебра изоморфна полиномиальной алгебре $\mathbb{Z} / 2\left[\xi_{1}, \ldots, \xi_{k}, \ldots\right]$, $\operatorname{deg} \xi_{k}=2^{k}-1$, а при $p>2$ она изоморфна тензорному произведению внешней и полиномиальной алгебр $E\left(\tau_{0}, \tau_{1}, \ldots, \tau_{l}, \ldots\right) \otimes \mathbb{Z} / p\left[\xi_{1}, \ldots, \xi_{k}, \ldots\right], \operatorname{deg} \xi_{k}=2\left(p^{k}-1\right)$, $\operatorname{deg} \tau_{l}=2 p^{l}-1$. Копроизведение $\psi$ задается по формулам

$$
\psi\left(\xi_{k}\right)=\sum_{i+j=k} \xi_{j}^{p^{i}} \otimes \xi_{i}, \quad \psi\left(\tau_{l}\right)=\tau_{l} \otimes 1+\sum_{i+j=l} \xi_{j}^{p^{i}} \otimes \tau_{i} .
$$


Tеорема 8.10. Кодействие алгебры $\mathscr{A}_{*}$, двойственной $к$ алгебре Стинрода, на гомология группь кос $\mathrm{Br}_{m}, 1 \leqslant m \leqslant \infty$, задается при $p=2$ по формуле:

$$
\psi\left(a_{j}\right)= \begin{cases}1 \otimes a_{j}, & \text { если } j=1, \\ 1 \otimes a_{j}+\xi_{1} \otimes a_{j-1}^{2}, & \text { если } j \geqslant 2,\end{cases}
$$

а при $р>2$ по формулам

$$
\begin{aligned}
& \psi\left(a_{j}\right)= \begin{cases}1 \otimes a_{j}, & \text { если } j=1, \\
1 \otimes a_{j}+\tau_{0} \otimes b_{j-1}, & \text { если } j \geqslant 2,\end{cases} \\
& \psi\left(b_{j}\right)= \begin{cases}1 \otimes b_{j}, & \text { если } j=1, \\
1 \otimes b_{j}-\xi_{1} \otimes b_{j-1}^{p}, & \text { если } j \geqslant 2 .\end{cases}
\end{aligned}
$$

Пусть $\Omega_{0}^{2} S^{2}$ есть компонента связности тривиальной петли в двукратном пространстве петель $\Omega^{2} S^{2}$. Г. Сегал установил следующую связь между группой кос от бесконечного числа нитей и итерированными пространствами петель [36].

Tеорема 8.11. Существует отображение $K\left(B r_{\infty}, 1\right) \rightarrow \Omega_{0}^{2} S^{2}$, индуцирующее изоморфизм в гомологиях для любой группь коэффиииентов $G$ (с тривиальным действием $\mathrm{Br}_{\infty}$ на $\left.G\right)$ :

$$
H_{*}\left(B r_{\infty}, G\right) \cong H_{*}\left(\Omega_{0}^{2} S^{2}, G\right)
$$

НАБРОСОК ДОКАЗАТЕЛЬСТВА. Имеется естественное отображение

$$
j: B\left(\mathbb{R}^{2}, k\right) \rightarrow \Omega_{k}^{2} S^{2},
$$

определенное следуюшим образом. Сначала мы заменяем пространство $B\left(\mathbb{R}^{2}, k\right)$ на гомотопически эквивалентное пространство конфигураций из $k$ непересекающихся дисков на плоскости. Теперь для данной конфигурации $\mathbb{D}=\left\{D_{1}, \ldots, D_{k}\right\}$ из $k$ непресекающихся дисков определим отображение

$$
f_{\mathbb{D}}: S^{2} \rightarrow S^{2}
$$

Рассмотрим область определения $f_{\mathbb{D}}$ как $\mathbb{R}^{2} \cup \infty, f_{\mathbb{D}}$ отображает дополнение к $D_{1} \cup \cdots \cup D_{k}$ в базисную точку $S^{2}$, а на диске $D_{i}$ является стандартным отождествлением $D_{i} / \partial D_{i}$ с $S^{2}$. Тогда

$$
\mathbb{D} \mapsto f_{\mathbb{D}}
$$

дает требуемое отображение

$$
j: B\left(\mathbb{R}^{2}, k\right) \rightarrow \Omega_{k}^{2} S^{2} .
$$

Осталось только доказать тот факт, что $j$ есть асимптотическая гомологическая эквивалентность.

Классическое расслоение Хопфа $S^{3} \rightarrow S^{2}$ индуцирует гомотопическую эквивалентность $\Omega^{2} S^{3} \simeq \Omega_{0}^{2} S^{2}$. Это позволяет нам рассматривать пространства $\Omega^{2} S^{3}$ и $\Omega_{0}^{2} S^{2}$ как варианты плюс-конструкции для $K\left(B r_{\infty}, 1\right)$. 
Имеется следуюшее расслоение пространства путей

$$
\Omega^{2} S^{3} \rightarrow P \Omega S^{3} \rightarrow \Omega S^{3}
$$

где $P \Omega S^{3}$ есть пространство путей над $\Omega S^{3}$. П. Гринберг и В. Сержиеску [75] построили ациклическую группу $A$ и группу $F^{\prime}$ такие, что следующая коммутативная диаграмма плюс-конструкций

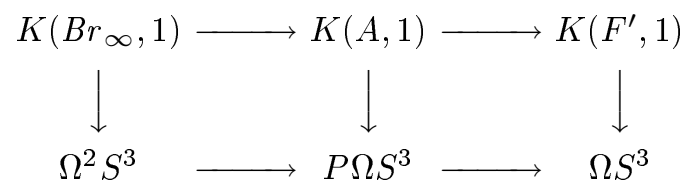

коммутативна.

П. Мэй доказал, что пространство $F\left(\mathbb{R}^{n}, m\right) \Sigma_{m}$-эквивариантно гомотопически эквивалентно пространству $\mathscr{C}_{n}(m)$ операды маленьких кубиков [26]. Эта операда играет ключевую роль в принципе распознавания $n$-кратных пространств петель (см. дополнение). Гомологии конфигурационных пространств использовались в конструкции операший Араки-Кудо-Даера-Лашофа, действуюших в гомологиях $n$-кратных пространств петель [32].

Э. Даер и Р. Лашоф [76], а также Ф.Р. Коэн [32; с. 227] изучали гомологии $\Omega^{n} S^{n+1}$. Из их результатов следует, что $H_{*}\left(\Omega^{n} S^{n+1}, \mathbb{Z} / p\right)$ изоморфно полиномиальной алгебре над $\mathbb{Z} / p$ от образующих $Q_{1}^{k_{1}} \ldots Q_{n-1}^{k_{n-1}} a_{1}$, где $Q_{i}$ суть операции Араки-Кудо-ДаераЛашофа, а $a_{1}$ есть образ порождаюшей $H_{1}\left(S^{1}, \mathbb{Z} / p\right)$ при отображении $H_{*}\left(S^{1}, \mathbb{Z} / p\right) \rightarrow$ $H_{*}\left(\Omega^{n} S^{n+1}, \mathbb{Z} / p\right)$, индуцированном каноническим вложением $S^{1} \rightarrow \Omega^{n} S^{n+1}$. Это дает другое доказательство теорем 8.4 и 8.8. Образующие $a_{j}, j>1$, можно считать равными $Q_{1}^{j-1} a_{1}, b_{j}$ равными $\beta a_{j+1}$. Кодействие двойственной к алгебре Стинрода, описанное в теореме 8.10, может быть получено из теоремы 8.11 с помошью соотношений Нишиды [32], связьваюших действие операций Стинрода и операций Араки-Кудо-Даера-Лашофа.

$\Phi$. Р. Коэн использовал спектральную последовательность Бокштейна для вычисления целочисленных гомологий групп кос [32].

Tеорема 8.12. B группах $H_{*}\left(B r_{n}, \mathbb{Z}\right), n \leqslant \infty, p$-кручение имеет порядок $p$. $B$ группах $H_{*}\left(B r_{\infty}, \mathbb{Z}\right)$ в размерностях, строго больиих единиць, р-кручение является следующим:

(i) если $p=2$, то это есть полиномиальная алгебра, порожденная $a_{1}$ и $a_{j}^{2}$, $j>1$;

(ii) если $p>2$, то әто есть тензорное произведение внешней алгебры, порожденной $a_{1}$, и полиномиальной алгебрьи, порожденной $b_{j}$.

Теорема 8.13. Каноническое вложсение $\mathrm{Br}_{n} \rightarrow \mathrm{Br}_{\infty}$ индучирует мономорфизм в гомологиях с коэффичиентами в $\mathbb{Z}$. Образ его 2-кручения в размерностях, строго больших 1 , имеет $\mathbb{Z} / 2$-базис, состоящий из мономов

$$
a_{1}^{k_{1}} \ldots a_{l}^{k_{l}} \text { maкux, чmo } k_{i} \equiv 0 \quad(\bmod 2) \quad \text { npu } i>1 \quad u \sum_{i} k_{i} 2^{i} \leqslant n \text {, }
$$


а образ его р-кручения при $p>2$ в размерностях, строго больиих 1, имеет $\mathbb{Z} / p$-базис, состоящий из мономов

$$
a_{1}^{\varepsilon_{1}} b_{1}^{k_{1}} \ldots b_{s}^{k_{s}}, \quad \text { де } \varepsilon_{1}=0,1 ; \quad u \quad 2\left(\varepsilon_{1}+\sum_{j} k_{j} p^{j}\right) \leqslant n .
$$

$\Phi$. В. Вайнштейн применил методы Д. Б. Фукса для вычисления когомологий групп кос с коэффициентами в $\mathbb{Z} / p$ и $\mathbb{Z}[33]$. В качестве результата он получил полную информацию об аддитивной структуре этих групп когомологий и о действии гомоморфизма Бокштейна.

Представление Кокстера симметрической группы $\Sigma_{n}$ есть представление

$$
\Sigma_{n} \rightarrow G L_{n}(\mathbb{Z})
$$

соответствуюшее перестановкам базисных векторов в $\mathbb{Z}^{n}$. Ограничение его на гиперплоскость $\mathbb{Z}^{n}$, заданную по формуле $\sum x_{i}=0$, назьвается приведенным представлением Кокстера. Эти представления определяют структуры $\Sigma_{n}$-модулей на $\mathbb{Z}^{n}$ и $\mathbb{Z}^{n-1}$. При помощи канонического гомоморфизма $B r_{n} \rightarrow \Sigma_{n}$ группы $\mathbb{Z}^{n}$ и $\mathbb{Z}^{n-1}$ становятся модулями над $B r_{n}$. Обозначим эти модули через $K_{n}$ и $\widetilde{K}_{n}$. В. А. Васильев доказал следующий факт [13].

ТЕОрема 8.14. Когомологии группь кос с коэффициентами в представлении Кокстера и в приведенном представлении Кокстера задаются по формулам:

$$
\begin{aligned}
H^{q}\left(B r_{n} ; K_{n}\right) & =\bigoplus_{i=0}^{n-1} H^{q-i}\left(B r_{n-1-i} ; \mathbb{Z}\right), \quad n \geqslant 2, \\
H^{q}\left(B r_{n} ; \widetilde{K}_{n}\right) & =\bigoplus_{i=1}^{n-1} H^{q-i}\left(B r_{n-i} ; \mathbb{Z}\right), \quad n \geqslant 2,
\end{aligned}
$$

где мы формально полагаем $B r_{0}$ равной единичной группе, $B r_{0}=\{e\}$.

ДокаЗАтЕЛЬСтво. Рассмотрим первую формулу. Модуль $K_{n}$ изоморфен модулю $\operatorname{Coind}_{B r_{1, n}}^{B r_{n}} \mathbb{Z}$, коиндуцированному из тривиального модуля $\mathbb{Z}$ над $B r_{1, n}$, где подгруппа $B r_{1, n}$ была определена в $\S 3$. По лемме Шапиро [77] имеем: $H^{*}\left(B r_{n}, K_{n}\right) \cong$ $H^{*}\left(B r_{1, n}, \mathbb{Z}\right)$. Докажем первьй изоморфизм нашего утверждения по индукции. Для $n=2$ группа $B r_{1,2}$ равна $\mathbb{Z}$ и, таким образом, ее когомологии такие же, как у окружности. Формула справедлива. Пусть $n>2$. Рассмотрим гомоморфизм $\beta: B r_{1, n} \rightarrow$ $B r_{n-1}$, определенньй в $\S 3$, и спектральную последовательность Серра-Хохшильда для $\beta$. Как это было описано в $\S 3, \operatorname{Ker} \beta$ есть свободная подгруппа $F_{n-1}$ групшы $B r_{n}$, порожденная косами $a_{2}, \ldots, a_{n}$. Начальньй член спектральной последовательности Серра-Хохшильда $E_{2}^{p, q}$ изоморфен $H^{p}\left(B r_{n-1}, H^{q}\left(F_{n-1}, \mathbb{Z}\right)\right)$. Имеются только две ненулевые строки в этой спектральной последовательности, так как

$$
H^{0}\left(F_{n-1}, \mathbb{Z}\right) \cong \mathbb{Z}, \quad H^{1}\left(F_{n-1}, \mathbb{Z}\right) \cong \mathbb{Z}^{n-1}, \quad H^{q}\left(F_{n-1}, \mathbb{Z}\right) \cong 0 \text { при } q>1 .
$$

Напомним, что группа $B r_{1, n}$ изоморфна полупрямому произведению $F_{n-1}$ и $B r_{n-1}$ со стандартным действием $B r_{n-1}$ на $F_{n-1}$. Это действие порождает на $H^{0}\left(F_{n-1}, \mathbb{Z}\right) \cong$ 
$\mathbb{Z}$ структуру тривиального $\mathbb{Z} B r_{n-1}$-модуля, а на $H^{1}\left(F_{n-1}, \mathbb{Z}\right) \cong \mathbb{Z}^{n-1}$ оно порождает структуру модуля $K_{n-1}$. Это заканчивает шаг индукции. Изоморфизм для приведенного представления Кокстера следует из точной последовательности $0 \rightarrow \widetilde{K}_{n} \rightarrow$ $K_{n} \rightarrow \mathbb{Z} \rightarrow 0$.

Н.С. Маркарян продолжил работу В.А. Васильева и выгислил когомологии кос с другими нетривиальными коэффициентами [78].

\section{§. Когомологии обобшенных групп кос}

В. В. Горюнов применил методы Д. Б. Фукса и Ф.В. Вайнштейна для вычисления когомологий обобшенных групп кос типов $C$ и $D$ [34], [35]. Конфигурационное пространство $X_{C_{n}}$ для групшы кос типа $C$ было описано в $\oint 3$ как пространство $n$ различных пар точек из $\mathbb{C} \backslash 0$, симметричных по отношению к нулю, что есть не что иное, как просто пространство $n$ различных точек из $\mathbb{C} \backslash 0$. Конфигурационное пространство $X_{D_{n}}$ для групшы кос типа $D$ может быть описано следуюшим образом. Рассмотрим геометрически различные пары точек из $\mathbb{C}$, симметричные относительно нуля. Вырожденньй случай, когда пара состоит из одной точки, равной нулю, включается. Предположим, что каждая невырожденная пара рассматривается с различными знаками точек (плюсом или минусом). Инволюция $\nu$ действует на невырожденных парах переменой знаков и тождественна на вырожденной паре. Два неупорядоченных множества из $n$ различных пар точек $\left\{p_{1}, \ldots, p_{n}\right\}$ и $\left\{q_{1}, \ldots, q_{n}\right\}$ эквивалентны, если $q_{i}=\nu^{\varepsilon_{i}}\left(p_{i}\right), \varepsilon_{i}=0,1$, и $\sum_{i=1}^{i=n} \varepsilon_{i}$ четно. Пространство $X_{D_{n}}$ есть фактор-пространство $n$-ок геометрически различных пар из $\mathbb{C}$ по отношению к только что описанному отношению эквивалентности.

Пространство $X_{A_{n-1}}=B(\mathbb{C}, n)$ в предыдущем параграфе было интерпретировано как пространство полиномов (8.1) степени $n$ над комплексными числами, без кратных корней и со старшим коэффициентом, равным 1. Аналогично интерпретируем пространство $X_{C_{n}}$ как пространство многочленов над $\mathbb{C}_{\text {вида }}(8.1)$ без кратных и нулевых корней. Пусть $\Gamma_{n}$ есть одно из пространств $X_{C_{n}}$ или $X_{D_{n}}$, а $\Gamma_{n}^{*}$ - его одноточечная компактификация. Так же, как Д. Б. Фукс, В. В. Горюнов использовал двойственность Пуанкаре

$$
H^{k}\left(\Gamma_{n}, \mathbb{Z}\right) \cong \widetilde{H}_{2 n-k}\left(\Gamma_{n}^{*}, \mathbb{Z}\right)
$$

для выгисления когомологий обобщенных групп кос типов $C$ и $D$. Он построил клеточное разбиение пространства $\Gamma_{n}^{*}$ и доказал следуюшие теоремы.

Теорема 9.1. Когомологии предельньх обобщенньх групп кос типов $C$ и $D$ с коэффичиентами в $\mathbb{Z}$ выражаются в терминах когомологий классических групп следующим образом:

$$
\begin{aligned}
& H^{q}\left(B r\left(C_{\infty}\right) ; \mathbb{Z}\right)=\bigoplus_{i=0}^{q} H^{q-i}\left(B r_{\infty} ; \mathbb{Z}\right), \\
& H^{q}\left(B r\left(D_{\infty}\right) ; \mathbb{Z}\right)=H^{q}\left(B r_{\infty} ; \mathbb{Z}\right) \oplus\left[\bigoplus_{i=0}^{\infty} H^{q-2 i-3}\left(B r_{\infty} ; \mathbb{Z} / 2\right)\right] .
\end{aligned}
$$


Обозначим через $\gamma_{n}$ каноническое вложение $\gamma_{n}: B r_{n-1} \rightarrow B r_{n}$, а через $\gamma_{n}^{q}$ гомоморфизм, индуцированный $\gamma_{n}$ в когомологиях:

$$
\gamma_{n}^{q}: H^{q}\left(B r_{n}, \mathbb{Z}\right) \rightarrow H^{q}\left(B r_{n-1}, \mathbb{Z}\right)
$$

$\operatorname{Ker} \gamma_{n}^{q}$ обозначает, как обычно, ядро этого гомоморфизма.

Теорема 9.2. Когомологии с коэффициентами в $\mathbb{Z}$ обобщенных групп кос $\operatorname{Br}\left(C_{n}\right)$ u $\operatorname{Br}\left(D_{n}\right)$ для конечного $n$ выражсаются в терминах когомологий классических групп следующим образом:

$$
\begin{aligned}
H^{q}\left(B r\left(C_{n}\right) ; \mathbb{Z}\right)=\bigoplus_{i=0}^{n} H^{q-i}\left(B r_{n-i} ; \mathbb{Z}\right), \quad n \geqslant 2, \\
H^{q}\left(B r\left(D_{n}\right) ; \mathbb{Z}\right)=H^{q}\left(B r_{n} ; \mathbb{Z}\right) \oplus\left[\bigoplus_{i=0}^{\infty} \operatorname{Ker} \gamma_{n-2 i}^{q-2 i}\right] \\
\oplus\left[\bigoplus_{j=0}^{\infty} H^{q-2 j-3}\left(B r_{n-2 j-3} ; \mathbb{Z} / 2\right)\right], \quad n \geqslant 3,
\end{aligned}
$$

где мы полагаем формально $B r_{0}$ равной единичной группе, $B r_{0}=\{e\}$.

Формула для когомологий $\operatorname{Br}\left(C_{n}\right)$ была доказана в предыдушем параграфе (теорема 8.14), так как

$$
H^{q}\left(B r\left(C_{n}\right) ; \mathbb{Z}\right)=H^{q}\left(B r_{1, n+1} ; \mathbb{Z}\right)=H^{q}\left(B r_{n} ; \widetilde{K}_{n}\right)
$$

СлЕДСТВИЕ 9.1 (теорема стабилизации). С возрастанием $n$ группь когомологий обобщенных групп кос типов $C$ и $D$ стабилизируются:

$$
\begin{array}{lll}
H^{i}\left(B r\left(C_{n}\right), \mathbb{Z}\right)=H^{i}\left(\operatorname{Br}\left(C_{2 i-2}\right), \mathbb{Z}\right), & \text { ecлu } & n \geqslant 2 i-2, \\
H^{i}\left(\operatorname{Br}\left(D_{n}\right), \mathbb{Z}\right)=H^{i}\left(\operatorname{Br}\left(D_{2 i-1}\right), \mathbb{Z}\right), & \text { еслu } & n \geqslant 2 i-1 .
\end{array}
$$

Д. Б. Фукс установил [68] аналоги теоремы Г. Сегала о плюс-конструкции для классифицируюшего пространства групшы кос от бесконечного числа нитей (теорема 8.11): плюс-конструкция для $K\left(B r\left(C_{\infty}\right), 1\right)$ эквивалентна $\Omega^{2} S^{3} \times \Omega S^{2}$, а плюс-конструкция для $K\left(\operatorname{Br}\left(D_{\infty}\right), 1\right)$ эквивалентна $\Omega^{2} S^{3} \times S^{3}\{2\}$, где $S^{3}\{2\}$ есть гомотопический слой отображения степени 2 из $S^{3}$ в $S^{3}$.

\section{$\S$ 10. Порядковые комплексы и когомологии исключительных обобщенных групп кос}

Пусть $M_{1}, M_{2}, \ldots, M_{m} \subset V$ есть конечное множество аффинных подпространств, возможно различных размерностей, $n$-мерного аффиинного пространства, и пусть

$$
Y_{V}=V-\bigcup_{i=1}^{m} M_{i}
$$


есть дополнение к объединению этих подпространств. С нашим семейством подпространств $\mathscr{M}$ ассоциировано частично упорядоченное множество $\mathscr{P}$, элементы $v$ которого соответствуют частично упорядоченным по включению пересечениям

$$
|v|=M_{i_{1}} \cap M_{i_{2}} \cap \cdots \cap M_{i_{r}}
$$

Единственньй максимальньй элемент $T$ этого множества соответствует объемлющему пространству $V$. Определим функцию размерности

$$
d(v)=\operatorname{dim}_{\mathbb{R}}(|v|) .
$$

Для любого частично упорядоченного множества $\mathscr{S}$ можно определить порядковыи комплекс $K(\mathscr{S})$ (см., например, [79]). Это есть симплициальный комплекс, имеющий по одной вершине для каждого элемента $v \in \mathscr{S}$ и по $k$-симплексу для каждой цепи $v_{0}<v_{1}<\cdots<v_{k}$ элементов из $\mathscr{S}$. Граница этого $k$-симплекса состоит из всех соответствуюших подцепей. Таким образом, $K(\mathscr{S})$ является классифицируюшим пространством категории, определенной частично упорядоченным множеством $\mathscr{S}$. Отметим, что $K(\mathscr{S})=K\left(\mathscr{S}^{*}\right)$, где $\mathscr{S}^{*}$ - дуальное частично упорядоченное множество, получающееся из $\mathscr{S}$ обрашением отношения порядка. Если $\mathscr{S}$ имеет минимальный или максимальньй элемент, то $K(\mathscr{S})$ стягиваем. Будем предполагать, что $\mathscr{S}$ имеет единственньй максимальный элемент $T$. Для любых двух сравнимых элементов $v<w$ частично упорядоченного множества $\mathscr{S}$ определим следуюшие подмножества:

$$
\begin{aligned}
\mathscr{S}_{[v, w]} & =\{x \in \mathscr{S} \mid v \leqslant x \leqslant w\}, \\
\mathscr{S}_{(v, w)} & =\{x \in \mathscr{S} \mid v<x<w\}, \\
\mathscr{S}_{>v} & =\{x \in \mathscr{S} \mid x>v\} .
\end{aligned}
$$

Порядковые комплексы используются при описании гомологий дополнений к конфигурациям афффинных плоскостей [79], [80].

Теорема 10.1. Гомологии дополнения $Y_{V}$ задаются по формулам:

$$
H_{i}\left(Y_{V} ; \mathbb{Z}\right)=\bigoplus_{v \in \mathscr{P}} H^{n-d(v)-i-1}\left(K\left(\mathscr{P}_{>v}\right), K\left(\mathscr{P}_{(v, T)}\right) ; \mathbb{Z}\right)
$$

где нами принято соглашение $H^{-1}(\varphi, \varnothing ; \mathbb{Z})=\mathbb{Z}$.

Комплексные конфигурации гиперплоскостей в $\mathbb{C}^{n}$ можно рассматривать как вешественные конфигурации подпространств коразмерности 2 в вешественном аффинном пространстве размерности $2 n$. Следовательно, эта теорема дает описание гомологий крашеных обобшенных групп кос.

При изучении когомологий исключительных обобщенных групп кос мы следуем здесь работам М. Сальветти [41], [42].

Первым шагом является построение комплекса $Q$, двойственного стратификации $\mathbb{C}^{n}$, полученной из конфигурации гиперплоскостей. Он может быть реализован в исходном аффинном пространстве $V$. Ячейки стратификации образуют частично упорядоченное множество $\mathscr{P}: F>F^{\prime}$, если замькание $F$ содержит $F^{\prime}$. Геометрическая реализация комплекса $K(\mathscr{P})$ может быть вложена в пространство $V$ следующим 
образом. Выберем точку $v\left(F^{j}\right)$ внутри каждой $j$-комерной ячейки стратификации и рассмотрим симплексы

$$
s\left(F^{i_{0}}, \ldots, F^{i_{j}}\right)=\left\{\sum_{k=0}^{j} \lambda_{k} v\left(F^{i_{k}}\right): \sum \lambda_{k}=1, \lambda_{k} \in[0,1]\right\}
$$

где $F^{i_{k}}>F^{i_{k+1}}, k=0, \ldots, j-1$. Затем мы строим клеточньй блок-комплекс из этого симплищиального комплекса, склеивая несколько симплексов вместе, чтобы получить клетку. Клетка $e^{j}\left(F^{j}\right)$ размерности $j$, двойственная к $F^{j}$, получается как объединение $\bigcup s\left(F^{0}, \ldots, F^{j-1}, F^{j}\right)$ по всем цепочкам $F^{0}>\cdots>F^{j-1}>F^{j}$. Пусть $Q=\bigcup e^{j}\left(F^{j}\right)$, где объединение берется по всем ячейкам, а $Q_{i}$ обозначает $i$-мерньй остов $Q$. Определим комбинаторное расстояние между двумя вершинами $v$ и $v^{\prime}$ как минимальное число ребер в реберном пути, соединяющем $v$ и $v^{\prime}$. Для каждой клетки $e^{j}$ из $Q$ обозначим через $V\left(e^{j}\right)=Q_{0} \cap e^{j}$-мерный остов $e^{j}$.

ПРЕДЛОЖЕНИЕ 10.1. Для данных вершины $v \in Q_{0}$ иклетки $e^{j} \in Q$ существует единственная вериина $w\left(v, e^{i}\right) \in V\left(e^{i}\right)$ с наименьиим комбинаторным расстоянием до $v$ :

$$
d\left(v, w\left(v, e^{i}\right)\right)<d\left(v, v^{\prime}\right), \quad \text { ecлu } \quad v^{\prime} \in V\left(e^{i}\right) \backslash\left\{w\left(v, e^{i}\right)\right\}
$$

Ecли $e^{j} \subset e^{i}$, mo $w\left(v, e^{j}\right)=w\left(w\left(v, e^{i}\right), e^{j}\right)$.

Зафиксируем камеру $A_{0}$, и пусть $v_{0}$ есть вершина из $Q$, содержашаяся в $A_{0}$. Пусть $\mathscr{F}_{0}$ - система ячеек $A_{0}$, а $\mathscr{Q}_{0}$ есть множество клеток в $Q$, двойственных к ячейке из $\mathscr{F}_{0}$, т.е. клеток, которые пересекаются с $A_{0}$. Заметим, что $\mathscr{Q}_{0}$ не является клеточным комплексом. Для данной клетки $e \in Q$ мы будем обозначать для краткости $w_{0}:=$ $w\left(v_{0}, e\right)$.

Чтобы определить действие группы $W$ на комплексе $Q$, проделаем построение более аккуратно. Выберем точку $v(F)$ внутри каждой ячейки $F \in \mathscr{F}_{0}$; внутри ячейки $\gamma(F), \gamma \in W$, выбираем точку $\gamma(v(F))$. Поскольку стабилизатор ячейки оставляет неподвижной ячейку поточечно, эта точка не зависит от выбора элемента $\gamma \in W$. Затем построим $Q$, как и вьше, используя эти точки. Ясно, что $Q$ и его остовы $Q_{i}$ инвариантны по отношению к $W$.

ПРЕДЛОЖЕНИЕ 10.2. Для каждой клетки е из $Q$ существует в точности единственная клетка $e_{0} \in \mathscr{Q}_{0}$ в фиксированной орбите действия $W$. Элементь $\gamma \in W$ такие, что $\gamma\left(e_{0}\right)=е$, описьвают левые смезсные классы стабилизатора $W_{F_{0}}$ ячейки $F_{0}$, әде $F_{0}$ двойственна $\kappa e_{0}$.

СлЕДСТВИЕ 10.1. Для клетки $е \in Q$ единственный әлемент $\gamma_{e} \in W$ такой, что $\gamma_{e}\left(v_{0}\right)=w_{0}(e)$, удовлетворяет также условию: $\gamma_{e}\left(e_{0}\right)=e$.

Построим комплекс $S_{W}$, проводя следующие отождествления клеток комплекса $Q$. Отождествим две клетки $e, e^{\prime} \in Q$, если они лежат в одной $W$-орбите, используя гомеоморфизм, индуцированньй элементом $\gamma_{e^{\prime}}\left(\gamma_{e}\right)^{-1}$. Пусть $Y$ - дополнение к вешественной конфигурации гиперплоскостей в $\mathbb{C}^{n}($ как в $\S 3)$. 
ТЕОрема 10.2. Пространство орбит $Y / W$ имеет тот же гомотопический тип, что и клеточныц комплекс $S_{W}$.

СЛЕДСТВИЕ 10.2. Если группа $W$ конечна и существенна, то число $i$-клеток $S_{W}$ ecmb $\left(\begin{array}{c}n \\ i\end{array}\right), i=0,1,2, \ldots, n$.

СлЕДСТВИЕ 10.3. Если әруппа $W$ конечна, то клеточный комплекс $S_{W}$ есть пространство типа $K\left(\pi_{1}(Y / W), 1\right)$.

Если выбрать точку $v(F), F \in \mathscr{F}_{0}$, как ортогональную проекцию $v_{0}$ на $F$, то все клетки $Q$ становятся выпукльми полиэдрами в аффинном пространстве $V$. А $Q$ сам является вьпуклой оболочкой $W$-орбиты точки $v_{0}$. Таким образом, теорема 10.2 описывает гомотопический тип $Y / W$ в терминах комплекса, полученного из выпуклого полиэдра отождествлением его граней.

Предположим с этого момента, что $W$ конечна и существенна. Пусть гиперплоскости (стенки) $A_{0}$ суть $M_{1}, \ldots, M_{n}$. Пусть также $v_{i} \in M_{i} \cap \bar{A}_{0}$ - точки из $Q, i=$ $1,2, \ldots, n$, выбранные, скажем, как ортогональные проекции $v_{0}$ на каждую $M_{i}$. Всякая ячейка $F$ соответствует единственному пересечению $M_{i_{1}} \cap \cdots \cap M_{i_{k}}, k=\operatorname{codim}(v)$, где $M_{i_{j}}$ являются стенками $A_{0}$, содержащими $F$, и $i_{1}<\cdots<i_{k}$. Следовательно, $F$ соответствует единственному подмножеству $\Gamma=\Gamma(F) \subset I_{n}=\{1, \ldots, n\}$, где $|\Gamma|=\operatorname{codim}(F)=\operatorname{dim} e(F)$. Клетки из $S_{W}$ соответствуют клеткам из $\mathscr{Q}_{0}$ и, таким образом, ячейкам $\mathscr{F}_{0}$ камеры $A_{0}$. Зададим на двойственной клетке $e(F)$ ориентацию, индуцированную упорядочиванием $v_{0}, v_{i_{1}}, \ldots, v_{i_{k}}$. На каждой клетке $e \in Q$ зададим ориентацию требованием, что каждьй элемент $\gamma_{e}$, описанный выше, эту ориентацию сохраняет. Тогда на клетках $Q$ определен индекс инцидентности $\left[e: e^{\prime}\right] \in\{0,1,-1\}$, и он сохраняется при переходе к фактор-комплексу $S_{W}$.

ПРЕДЛОЖЕНИЕ 10.3. Пусть $F \in \mathscr{F}_{0}$ coответствует подмножеству Г. Пусть также $G^{\prime}$ есть ячейка, $W$-эквивалентная ячейке $F^{\prime} \in \mathscr{F}_{0}$, соответствующей $\Gamma^{\prime} \subset \Gamma$, где $\left|\Gamma^{\prime}\right|=|\Gamma|-1$, и такая, что $\overline{G^{\prime}} \supset F$ (таким образом, $G^{\prime}$ $W_{F}$-əквивалентна $\left.F^{\prime}\right)$. Тогда $е(F) \supset e\left(G^{\prime}\right)$ и их индекс инцидентности есть

$$
\left[e(F): e\left(G^{\prime}\right)\right]=(-1)^{l\left(G^{\prime}\right)}\left[e(F): e\left(F^{\prime}\right)\right],
$$

где $l\left(G^{\prime}\right)$ есть кратчайшая длина $l(g)$ әлемента $g \in W$, переводящего $F^{\prime}$ в $G^{\prime}$.

ЗАмЕчАниЕ. Для числа $l\left(G^{\prime}\right)$ выполняется равенство

$$
l\left(G^{\prime}\right)=d\left(v_{0}, w_{0}\left(e\left(G^{\prime}\right)\right)\right) .
$$

Пусть $\mathscr{C}^{k}$ есть свободньй $\mathbb{Z}$-модуль, порожденный $k$-клетками $S_{W}, k=0, \ldots, n$. Из предыдушего следует, что

$$
\mathscr{C}^{k} \cong\left\{\sum \nu_{\Gamma} \Gamma: \nu_{\Gamma} \in \mathbb{Z}, \Gamma \subset I_{n},\left|I_{n}\right|=k\right\} .
$$

Определим кограничньй гомоморфизм $\delta^{k}: \mathscr{C}^{k} \rightarrow \mathscr{C}^{k+1}$ в градуированном модуле $\mathscr{C}$, порожденном $\mathscr{C}^{k}$ :

$$
\delta^{k}(\Gamma)=\sum_{j \in I_{n} \backslash \Gamma}(-1)^{\sigma(j, \Gamma)+1}\left(\sum_{\underline{h} \in W_{\Gamma \cup\{j\}} / W_{\Gamma}}(-1)^{l(\underline{h})}(\Gamma \cup\{j\})\right),
$$

где положено $\sigma(j, \Gamma)=|\{i \in \Gamma: i<j\}|, W_{\Gamma}=W_{\Gamma(F)}=W_{F}$ и $l(\underline{h})$ есть минимальная длина элемента $h \in \underline{h}$. 
ТЕОРема 10.3. Когомологии комплекса $S_{W}$ изоморфны когомологиям комплекса $\mathscr{C}$ :

$$
H^{*}\left(S_{W} ; \mathbb{Z}\right) \cong H^{*}(\mathscr{C} ; \mathbb{Z})
$$

Эта теорема позволяет легко посчитать когомологии всех обобщенных групп кос, в том числе исключительные. Результаты для исключительных даны в таблице 10.1 из [42]. М. Сальветти вычислил также когомологии с локальньми коэффициентами.

ТАБЛ. 10.1

\begin{tabular}{|c|c|c|c|c|c|c|c|c|c|}
\hline & $H^{0}$ & $H^{1}$ & $H^{2}$ & $H^{3}$ & $H^{4}$ & $H^{5}$ & $H^{6}$ & $H^{7}$ & $H^{8}$ \\
\hline$I_{2}(2 s)$ & $\mathbb{Z}$ & $\mathbb{Z}^{2}$ & $\mathbb{Z}$ & & & & & & \\
\hline$I_{2}(2 s+1)$ & $\mathbb{Z}$ & $\mathbb{Z}$ & & & & & & & \\
\hline$H_{3}$ & $\mathbb{Z}$ & $\mathbb{Z}$ & $\mathbb{Z}$ & $\mathbb{Z}$ & & & & & \\
\hline$H_{4}$ & $\mathbb{Z}$ & $\mathbb{Z}$ & 0 & $\mathbb{Z} \times \mathbb{Z} / 2$ & $\mathbb{Z}$ & & & & \\
\hline$F_{4}$ & $\mathbb{Z}$ & $\mathbb{Z}^{2}$ & $\mathbb{Z}^{2}$ & $\mathbb{Z}^{2}$ & $\mathbb{Z}$ & & & & \\
\hline$E_{6}$ & $\mathbb{Z}$ & $\mathbb{Z}$ & 0 & $\mathbb{Z} / 2$ & $\mathbb{Z} / 2$ & $\mathbb{Z} / 6$ & $\mathbb{Z} / 3$ & & \\
\hline$E_{7}$ & $\mathbb{Z}$ & $\mathbb{Z}$ & 0 & $\mathbb{Z} / 2$ & $\mathbb{Z} / 2 \times \mathbb{Z} / 2$ & $\mathbb{Z} / 6 \times \mathbb{Z} / 6$ & $\mathbb{Z} / 3 \times \mathbb{Z} / 6 \times \mathbb{Z}$ & $\mathbb{Z}$ & \\
\hline$E_{8}$ & $\mathbb{Z}$ & $\mathbb{Z}$ & 0 & $\mathbb{Z} / 2$ & $\mathbb{Z} / 2$ & $\mathbb{Z} / 2 \times \mathbb{Z} / 6$ & $\mathbb{Z} / 3 \times \mathbb{Z} / 6$ & $\mathbb{Z} / 2 \times \mathbb{Z} / 6 \times \mathbb{Z}$ & $\mathbb{Z}$ \\
\hline
\end{tabular}

\section{§11. Гомологии групп кос в телах с ручками и гомологии конфигурационных пространств для поверхностей}

В этом параграфе мы изучаем гомологические свойства группы кос от бесконечного числа нитей в теле с ручками рода $g$ и конфигурационного пространства из бесконечного числа точек на поверхности рода $h$. Мы выражаем эти свойства в терминах плюс-конструкции (определение плюс-конструкции см. в $\S 6$ ).

ТеОРема 11.1. Плюс-конструкиия Д. Квиллена классифицирующего пространства группы кос из бесконечного числа нитей в теле с ручками рода $g$ әквивалентна следующему произведению пространств петель над сферами

$$
B B r_{\infty}^{g}+\simeq \Omega^{2} S^{3} \times \Omega S^{2} \times \cdots \times \Omega S^{2}
$$

где число сомножителей в произведении равно роду $g$. Диаграмма

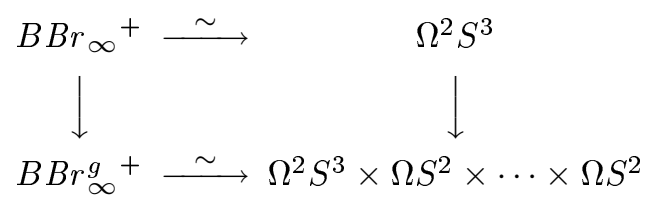

в которой левая стрелка индуцирована каноническим вложением классической группь кос в группу кос в теле с ручками, а правая стрелка есть вложение сомножителя, коммутативна. 
ДокАЗАТЕльСтво. Для наших целей будет удобно рассматривать конфигурационное пространство $B\left(\mathbb{C} \backslash Q_{g}, n\right)$ следуюшим образом. Пусть $D$ обозначает замкнутый диск в $\mathbb{C}$, а $D_{\varepsilon}^{i}, i=1, \ldots, g$, суть маленькие замкнутые диски в $D$. Тогда $\mathbb{C} \backslash Q_{g}$ гомеоморфно $\dot{D} \backslash\left(\bigcup_{i=1}^{g} D_{\varepsilon}^{i}\right)$, где $\dot{D}$ обозначает внутренность $D$. Таким образом, нужное конфигурационное пространство есть $B\left(\dot{D} \backslash\left(\bigcup_{i=1}^{g} D_{\varepsilon}^{i}\right), n\right)$. Мы используем здесь результаты Д. Мак-Дуфф [81]. Она рассматривала расслоение $E_{M}$ над многообразием $M$, которое получается одноточечной компактификацией каждого слоя касательного расслоения. Пусть $\Gamma(M)$ есть пространство сечений расслоения $E_{M}$ с компактными носителями, а $\Gamma(M, \partial M)$ составляют сечения $E_{M}$, обрашаюшиеся в нуль на краю $\partial M$. Можно определить понятие степени для таких сечений. Обозначим через $\Gamma_{n}(M)$ пространство, состолшее из сечений степени $n$. Д. Мак-Дуфф доказала, что если $\dot{M}$ есть внутренность компактного многообразия с краем, то существует отображение

$$
B(\dot{M}, n) \rightarrow \Gamma_{n}(M, \partial M)
$$

такое, что соответствуюший гомоморфизм в гомологиях

$$
H_{k}(B(\dot{M}, n) ; \mathbb{Z}) \rightarrow H_{k}\left(\Gamma_{n}(M, \partial) ; \mathbb{Z}\right)
$$

является изоморфизмом для больших $n$. Пусть $\gamma_{i}, i=1, \ldots, g$, есть простая кривая, лежашая в $D \backslash\left(\bigcup_{i=1}^{g} \dot{D}_{\varepsilon}^{i}\right)$, соединяюшая край диска $D$ с краем диска $D_{\varepsilon}^{i}$. Предположим, что кривые $\gamma_{i}$ не пересекаются, как это показано на рис. 11.1.

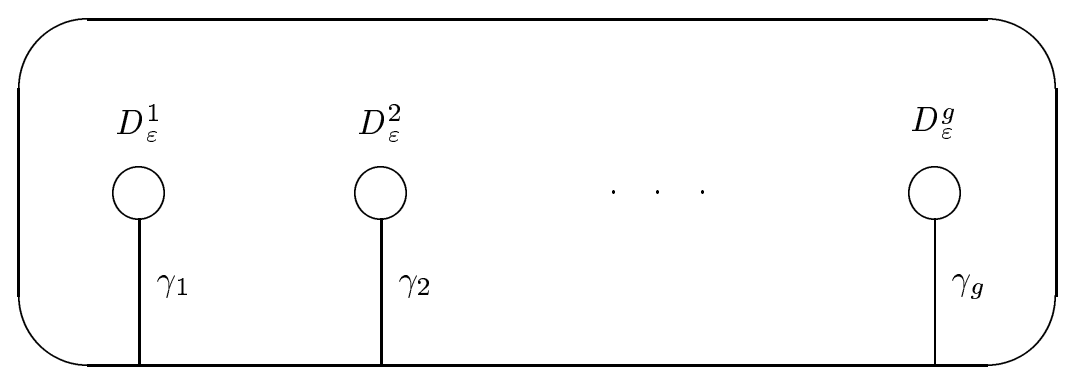

Рис. 11.1

Тогда пространство $\dot{D} \backslash\left(\left(\bigcup_{i=1}^{g} D_{\varepsilon}^{i}\right) \cup\left(\bigcup_{i=1}^{g} \gamma_{i}\right)\right)$ гомеоморфно внутренности некоторого диска, скажем $\dot{D}^{\prime}$. Гомеоморфизм вложения $\dot{D}^{\prime} \rightarrow \dot{D} \backslash\left(\left(\bigcup_{i=1}^{g} D_{\varepsilon}^{i}\right) \cup\left(\bigcup_{i=1}^{g} \gamma_{i}\right)\right)$ порождает следуюшую коммутативную диаграмму

$$
\begin{aligned}
& B\left(\dot{D}^{\prime}, n\right) \quad \longrightarrow \quad \Gamma_{n}\left(D^{\prime}, \partial D^{\prime}\right) \\
& B\left(\dot{D} \backslash\left(\bigcup_{i=1}^{g} D_{\varepsilon}^{i}\right), n\right) \longrightarrow \Gamma_{n}\left(D \backslash\left(\bigcup_{i=1}^{g} \dot{D}_{\varepsilon}^{i}\right), \partial D \cup\left(\bigcup_{i=1}^{g} \partial D_{\varepsilon}^{i}\right)\right)
\end{aligned}
$$


Для наших многообразий $D^{\prime}$ и $D \backslash\left(\bigcup_{i=1}^{g} \dot{D}_{\varepsilon}^{i}\right)$ касательные расслоения эквивалентны прямым произведениям, следовательно, соответствующие расслоения $E_{M}$ эквивалентны $S^{2} \times D^{\prime}$ и $S^{2} \times D \backslash\left(\bigcup_{i=1}^{g} \dot{D}_{\varepsilon}^{i}\right)$. Таким образом, пространства $\Gamma(M, \partial M)$ эквивалентны

$$
\operatorname{Map}\left(D^{\prime}, \partial D^{\prime} ; S^{2}, s_{0}\right) \text { и } \operatorname{Map}\left(D \backslash\left(\bigcup_{i=1}^{g} \dot{D}_{\varepsilon}^{i}\right), \partial D \cup\left(\bigcup_{i=1}^{g} \partial D_{\varepsilon}^{i}\right) ; S^{2}, s_{0}\right),
$$

где $s_{0}$ есть базисная точка сферы $S^{2}$. Вложение объединения кривых $\bigcup_{i=1}^{g} \gamma_{i}$ в многообразие $D \backslash\left(\bigcup_{i=1}^{g} \dot{D}_{\varepsilon}^{i}\right)$ порождает отображение соответствующих пространств отображений

$$
p: \operatorname{Map}\left(D \backslash\left(\bigcup_{i=1}^{g} \dot{D}_{\varepsilon}^{i}\right), \partial D \cup\left(\bigcup_{i=1}^{g} \partial D_{\varepsilon}^{i}\right) ; S^{2}, s_{0}\right) \rightarrow \operatorname{Map}\left(\bigcup_{i=1}^{g} \gamma_{i}, \bigcup_{i=1}^{g} \partial \gamma_{i} ; S^{2}, s_{0}\right) .
$$

Отображение $p$ является расслоением в смысле Серра со слоем

$$
\operatorname{Map}\left(D \backslash\left(\bigcup_{i=1}^{g} \dot{D}_{\varepsilon}^{i}\right), \partial D \cup\left(\bigcup_{i=1}^{g} \partial D_{\varepsilon}^{i}\right) \cup\left(\bigcup_{i=1}^{g} \gamma_{i}\right) ; S^{2}, s_{0}\right),
$$

которьй эквивалентен $\mathrm{Map}\left(D^{\prime}, \partial D^{\prime} ; S^{2}, s_{0}\right)$. Рассматривая связную компоненту

$$
\operatorname{Map}_{n}\left(D \backslash\left(\bigcup_{i=1}^{g} \dot{D}_{\varepsilon}^{i}\right), \partial D \cup\left(\bigcup_{i=1}^{g} \partial D_{\varepsilon}^{i}\right) ; S^{2}, s_{0}\right)
$$

пространства

$$
\operatorname{Map}\left(D \backslash\left(\bigcup_{i=1}^{g} \dot{D}_{\varepsilon}^{i}\right), \partial D \cup\left(\bigcup_{i=1}^{g} \partial D_{\varepsilon}^{i}\right) ; S^{2}, s_{0}\right),
$$

мы получим расслоение

$$
p_{n}: \operatorname{Map}_{n}\left(D \backslash\left(\bigcup_{i=1}^{g} \dot{D}_{\varepsilon}^{i}\right), \partial D \cup\left(\bigcup_{i=1}^{g} \partial D_{\varepsilon}^{i}\right) ; S^{2}, s_{0}\right) \rightarrow \operatorname{Map}\left(\bigcup_{i=1}^{g} \gamma_{i}, \bigcup_{i=1}^{g} \partial \gamma_{i} ; S^{2}, s_{0}\right) .
$$

Таким образом, из (11.1) получаем следуюшую диаграмму

$$
\begin{gathered}
B\left(\dot{D}^{\prime}, n\right) \\
\downarrow \\
B\left(\dot{D} \backslash\left(\bigcup_{i=1}^{g} D_{\varepsilon}^{i}\right), n\right) \longrightarrow \operatorname{Map}_{n}\left(D^{\prime}, \partial D^{\prime} ; S^{2}, s_{0}\right) \\
\downarrow
\end{gathered}
$$

в которой правое отображение есть вложение слоя расслоения $p_{n}$. Расслоение $p_{0}$ имеет сечение, определяемое следуюшим образом. Зафиксируем отображение из

$$
\operatorname{Map}\left(\bigcup_{i=1}^{g} \gamma_{i}, \bigcup_{i=1}^{g} \partial \gamma_{i} ; S^{2}, s_{0}\right)
$$


Делим многообразие $D \backslash\left(\bigcup_{i=1}^{g} \dot{D}_{\varepsilon}^{i}\right)$ на некоторые области $\widetilde{D}^{i}$, содержашие только по одному диску $D_{\varepsilon}^{i}$, как это показано на рис. 11.2 .

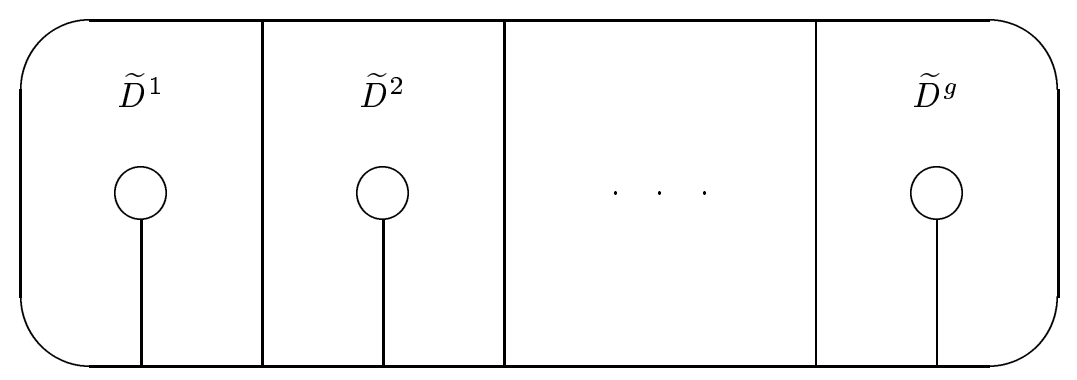

Рис. 11.2

Область $\widetilde{D}^{i}$ гомеоморфна произведению интервала $I$ и окружности $S^{1}$, таким образом, что $\gamma_{i}$ гомеоморфна некоторому радиусу. Мы отображаем каждый радиус в $S^{2}$, используя ранее фиксированное отображение из $\gamma_{i}$ в $S^{2}$, и получаем отображение, постоянное по $S^{1}$. Это определяет отображение

$$
r: \operatorname{Map}\left(\bigcup_{i=1}^{g} \gamma_{i}, \bigcup_{i=1}^{g} \partial \gamma_{i} ; S^{2}, s_{0}\right) \rightarrow \operatorname{Map}_{0}\left(D \backslash\left(\bigcup_{i=1}^{g} \dot{D}_{\varepsilon}^{i}\right), \partial D \cup\left(\bigcup_{i=1}^{g} \partial D_{\varepsilon}^{i}\right) ; S^{2}, s_{0}\right)
$$

такое, что $p_{0} \circ r=1$. Если мы сложим отображение $r$ с отображением замкнутого диска в $S^{2}$ степени $n$, мы получим отображение $r_{n}$, являюшееся сечением для $p_{n}$. Сушествование сечения дает гомотопические эквивалентности

$$
\begin{aligned}
& \operatorname{Map}_{n}\left(D \backslash\left(\bigcup_{i=1}^{g} \dot{D}_{\varepsilon}^{i}\right), \partial D \cup\left(\bigcup_{i=1}^{g} \partial D_{\varepsilon}^{i}\right) ; S^{2}, s_{0}\right) \\
& \simeq \operatorname{Map}_{n}\left(D^{\prime}, \partial D^{\prime} ; S^{2}, s_{0}\right) \times \operatorname{Map}\left(\bigcup_{i=1}^{g} \gamma_{i}, \bigcup_{i=1}^{g} \partial \gamma_{i} ; S^{2}, s_{0}\right) \\
& \simeq \Omega_{n}^{2} S^{2} \times \Omega S^{2} \times \cdots \times \Omega S^{2},
\end{aligned}
$$

где число сомножителей в произведении равно роду $g$.

СЛЕДСТВИЕ 11.1. Гомологии группы кос из бесконечного числа нитей в теле с ручками рода g изоморфнь

$$
H_{*}\left(B r_{\infty} ; \mathbb{Z}\right) \otimes H_{*}\left(\Omega S^{2} ; \mathbb{Z}\right)^{\otimes^{g}}
$$

Пусть $\Delta^{n}, \Delta_{k}^{n}, k=1, \ldots, g$, суть следуюшие “дискриминантные" отображения:

$$
\begin{aligned}
& \Delta^{n}: B B r_{\infty}^{g} \rightarrow \mathbb{C} \backslash 0 \cong S^{1}, \quad 2 \leqslant n \leqslant \infty \\
& \Delta_{k}^{n}: B B r_{\infty}^{g} \rightarrow \mathbb{C} \backslash 0 \cong S^{1}, \quad k=1, \ldots, g, \quad 2 \leqslant n \leqslant \infty
\end{aligned}
$$


которые определены для конечных $n$ на конфигурационных пространствах по формулам

$$
\Delta^{n}\left(z_{1}, \ldots, z_{n}\right)=\prod_{i \neq j}\left(z_{i}-z_{j}\right), \quad \Delta_{k}^{n}\left(z_{1}, \ldots, z_{n}\right)=\prod_{i}\left(z_{i}-k\right), \quad k=1,2, \ldots, g,
$$

а для $n=\infty$ как пределы. Тогда они определяют отображения

$$
a_{n}: B B r_{n}^{g} \rightarrow\left(S^{1}\right)^{\times^{g+1}}, \quad 2 \leqslant n \leqslant \infty
$$

такие, что $\pi_{1}\left(a_{n}\right)$ есть гомоморфизм абелеанизации группы $B r_{n}^{g}$.

Теорема 11.1 допускает обобщение на случай гомологий конфигурационных пространств для поверхностей, у которых удалены $k$ точек (или, что то же самое, для конфигурационных пространств поверхностей с $k$ компонентами края), если $k>0$. Пусть $M_{h}$ есть замкнутая поверхность рода $h$. Обозначим через $M_{h} \backslash Q_{k}$ пространство $M_{h}$ с удаленными $k$ точками. Пусть $Y_{h}$ есть пространство расслоения

$$
\Omega^{2} S^{3} \rightarrow Y_{h} \rightarrow\left(S^{1}\right)^{2 h},
$$

которое классифицируется отображением $\left(S^{1}\right)^{2 h} \rightarrow \Omega S^{3}$, переводяшим образующую групшы $H^{2}\left(\Omega S^{3}, \mathbb{Z}\right)$ в $2\left(\sum x_{2 i-1} x_{2 i}\right)$, где $x_{j}$ обозначает образующую группы $H^{1}\left(S^{1}, \mathbb{Z}\right)[68]$.

Теорема 11.2. Плюс-конструкиия Д. Квиллена бесконечного конфигурационного пространства для поверхности рода $h$ с удаленными $k$ точками әквивалентна следующему произведению пространств:

$$
B\left(M_{h} \backslash Q_{k}, \infty\right)^{+} \simeq\left(\Omega S^{2}\right)^{k-1} \times\left(\Omega S^{3}\right)^{2 h} \times Y_{h} .
$$

Диаграмма

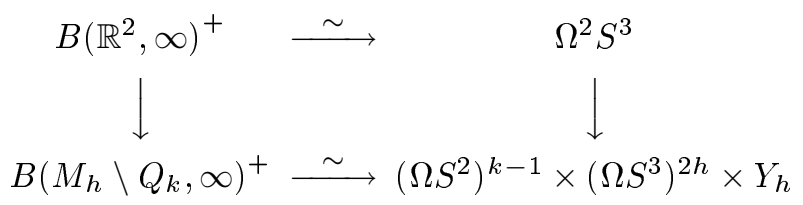

в которой левая стрелка индуцирована вложением конфигурационных пространств, а правая есть вложсение слоя $\Omega^{2} S^{3} \rightarrow Y_{h}$, коммутативна.

ДокаЗАтЕльство. Пусть $D_{\varepsilon}^{i}, i=1, \ldots, k$, суть маленькие непересекающиеся диски в $M_{h}$. Тогда пространство $M_{h} \backslash Q_{k}$ гомеоморфно $M_{h} \backslash \bigcup_{i=1}^{k} D_{\varepsilon}^{i}$. Многообразие $M_{h} \backslash Q_{k}$ параллелизуемо, если $k>0$, следовательно, остаются справедливыми те же самые аргументы, что и в доказательстве теоремы 11.1. Рассмотрим многоугольник $P_{h}$ такой, что поверхность $M_{h}$ получается из $P_{h}$ стандартной процедурой отождествления пар ребер. Пусть $P_{h}^{\prime}$ будет аналогичный многоугольник для поверхности без $k$ дисков $M_{h} \backslash \bigcup_{i=1}^{k} D_{\varepsilon}^{i}$ (см. рис. 11.3). Зафиксируем первый диск $D_{\varepsilon}^{1}$. Он будет играть роль внешней границы области, которая была в доказательстве теоремы 11.1. Делаем с многоугольником $P_{h}^{\prime}$ то же самое, что мы делали с диском $D$ в доказательстве теоремы 11.1. Соединяем некоторыми интервалами $\gamma_{i}, i=2, \ldots, k$, края дисков $D_{\varepsilon}^{i}$, $i=2, \ldots, k$, с краем диска $D_{\varepsilon}^{1}$. 


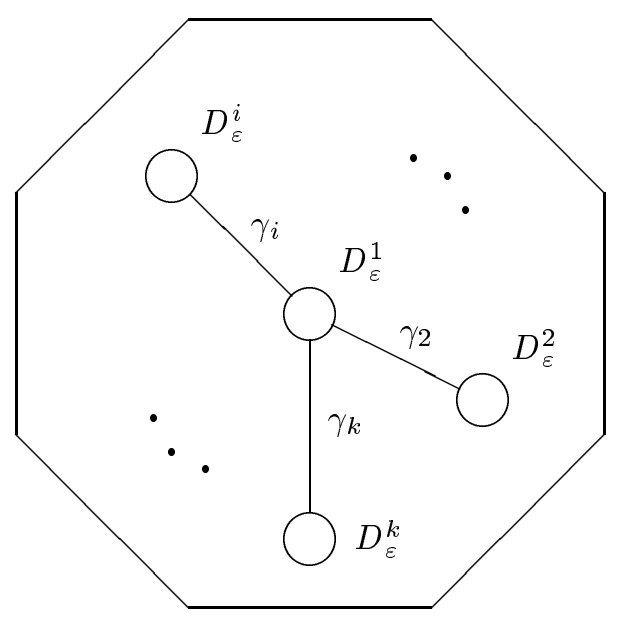

Рис. 11.3

Имеется эквивалентность

$$
B\left(M_{h} \backslash Q_{k}, n\right) \simeq B\left(M_{h} \backslash\left(\bigcup_{i=1}^{k} D_{\varepsilon}^{i}\right), n\right)
$$

и отображение

$$
B\left(M_{h} \backslash\left(\bigcup_{i=1}^{k} D_{\varepsilon}^{i}\right), n\right) \rightarrow \operatorname{Map}_{n}\left(M_{h} \backslash\left(\bigcup_{i=1}^{k} \dot{D}_{\varepsilon}^{i}\right), \bigcup_{i=1}^{k} \partial D_{\varepsilon}^{i} ; S^{2}, s_{0}\right),
$$

индуцирующее изоморфизм в гомологиях для больших $n$. Рассматривая последнее пространство, имеем также эквивалентности

$$
\begin{aligned}
\operatorname{Map}_{n}\left(M_{h} \backslash\left(\bigcup_{i=1}^{k} \dot{D}_{\varepsilon}^{i}\right), \bigcup_{i=1}^{k} \partial D_{\varepsilon}^{i} ; S^{2}, s_{0}\right) \\
\simeq \operatorname{Map}_{n}\left(M_{h, 1}, \partial M_{h, 1} ; S^{2}, s_{0}\right) \times \operatorname{Map}\left(\bigcup_{i=2}^{k} \gamma_{i}, \bigcup_{i=2}^{k} \partial \gamma_{i} ; S^{2}, s_{0}\right) \\
\simeq \operatorname{Map}_{n}\left(M_{h, 1}, \partial M_{h, 1} ; S^{2}, s_{0}\right) \times\left(\Omega S^{2}\right)^{k-1} \\
\simeq \operatorname{Map}_{n}\left(M_{h}, m_{0} ; S^{2}, s_{0}\right) \times\left(\Omega S^{2}\right)^{k-1}
\end{aligned}
$$

где $M_{h, 1}$ есть многообразие, получаюшееся из $M_{h}$ удалением открытого диска, а $m_{0} \in M_{h}$ - точка. Следовательно, имеется отображение

$$
B\left(M_{h} \backslash\left(\bigcup_{i=1}^{k} D_{\varepsilon}^{i}\right), \infty\right) \rightarrow \operatorname{Map}_{0}\left(M_{h}, m_{0} ; S^{2}, s_{0}\right) \times\left(\Omega S^{2}\right)^{k-1},
$$

индуцирующее изоморфизм в гомологиях. 
К.- Ф. Бедигхаймер, Ф. Р. Коэн и Р. Дж. Мильграм доказали [82] следуюшую гомотопическую эквивалентность:

$$
\operatorname{Map}_{0}\left(M_{h}, m_{0} ; S^{2}, s_{0}\right) \simeq\left(\Omega S^{3}\right)^{2 h} \times Y_{h} .
$$

Используя эту гомотопическую эквивалентность, мы завершаем доказательство теоремы.

СледСТвиЕ 11.2. Имеем следующий изоморфизм в гомологиях с коэффициентами в $\mathbb{Z} / 2$ :

$$
H^{*}\left(B\left(M_{h} \backslash Q_{k}, \infty\right) ; \mathbb{Z} / 2\right) \cong H^{*}\left(\Omega S^{2} ; \mathbb{Z} / 2\right)^{\otimes^{2 h+k-1}} \otimes H^{*}\left(\Omega^{2} S^{3} ; \mathbb{Z} / 2\right) .
$$

Диаграмма

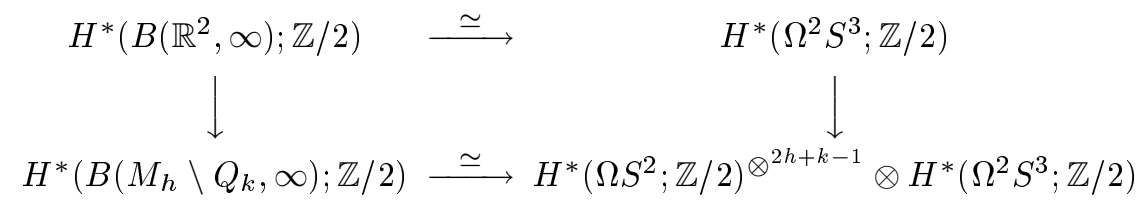

в которой левая стрелка индуцирована каноническим вложением конфигурационных пространств, коммутативна.

ЗАмечание. Результаты этого параграфа были получены в [33].

\section{$\S 12$. Гомологии кос с особенностями}

В этом параграфе мы рассматриваем группу кос-перестановок и группу кос с особенностями с гомологической точки зрения.

ПРЕДЛОЖЕнИЕ 12.1. Канонический эпиморфизм $B P_{\infty} \rightarrow \overline{B P}_{\infty}$ индуцирует отображсение групповых пополнений

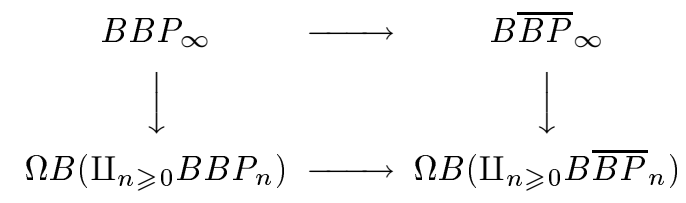

в котором нижняя строчка есть бесконечнократное петлевое отображение. Следовательно, имеются изоморфизмь

$$
B B P_{\infty}^{+} \cong \Omega_{0} B\left(\amalg_{n \geqslant 0} B B P_{n}\right), \quad B \overline{B P}_{\infty}^{+} \cong \Omega_{0} B\left(\amalg_{n \geqslant 0} B \overline{B P}_{n}\right),
$$

которые могут быть включены в коммутативную диаграмму, аналогичную (12.1).

Нам потребуется следующий простой факт о группах $B P_{n}$ и $\overline{B P}_{n}$. 
ПРЕДЛОЖЕНИЕ 12.2. Абелеанизации групп $B P_{n} u \overline{B P}_{n}, 2 \leqslant n \leqslant \infty$, равнь $\mathbb{Z} \oplus \mathbb{Z} / 2$ :

$$
\begin{aligned}
B P_{n} /\left[B P_{n}, B P_{n}\right] & \cong \mathbb{Z} \oplus \mathbb{Z} / 2, \\
\overline{B P}_{n} /\left[\overline{B P}_{n}, \overline{B P}_{n}\right] & \cong \mathbb{Z} \oplus \mathbb{Z} / 2 .
\end{aligned}
$$

ДокАЗАТЕльство. Добавим следуюшие соотношения к соотношениям группы кос $B r_{n}$ и симметрической группы $\Sigma_{n}: \sigma_{i}=\sigma_{j}$ для всех $i$ и $j, \xi_{i}=\xi_{j}$ для всех $i$ и $j$, и получим эпиморфизмы абелеанизации из группы кос и симметрической групшы $a b_{B r}: B r_{n} \rightarrow \mathbb{Z}$ и $a b_{\Sigma}: \Sigma_{n} \rightarrow \mathbb{Z} / 2$. Если добавим соотношения этих двух типов к определяюшим соотношениям группы кос-перестановок, получим эпиморфизм $a b_{B P}: B P_{n} \rightarrow \mathbb{Z} \oplus \mathbb{Z} / 2$. С другой стороны, универсальность дает то, что гомоморфизм абелеанизации для свободного произведения группы кос и симметрической группы $\mathrm{Br}_{n} * \Sigma_{n}$ может быть определен как композищия

$$
a b_{B P * \Sigma}: B r_{n} * \Sigma_{n} \stackrel{a b \quad * a b_{\Sigma}}{\longrightarrow} \mathbb{Z} * \mathbb{Z} / 2 \rightarrow \mathbb{Z} \oplus \mathbb{Z} / 2
$$

в которой второе отображение есть канонический эпиморфизм. Рассмотрим гомоморфизм $a b_{B} P * \Sigma$ как следующую композищию:

$$
B r_{n} * \Sigma_{n} \longrightarrow B P_{n} \stackrel{a b_{B P}}{\longrightarrow} \mathbb{Z} \oplus \mathbb{Z} / 2
$$

где первое отображение есть канонический эпиморфизм. Снова используя универсальность, видим, что $a b_{B P}$ есть абелеанизация $B P_{n}$. Доказательство для редуцированной группы кос-перестановок такое же.

Имеются эпиморфизмы $\alpha_{n}: B P_{n} \rightarrow \mathbb{Z}$ и $\bar{\alpha}_{n}: \overline{B P}_{n} \rightarrow \mathbb{Z}$, заданные по формулам

$$
\begin{gathered}
\xi_{i} \mapsto 0 \text { для всех } i, \\
\sigma_{i} \mapsto 1 \text { для всех } i .
\end{gathered}
$$

Из определяющих соотношений группы кос-перестановок следует, что мы имеем эпиморфизм $\phi_{n}: B P_{n} \rightarrow \Sigma_{n}$, определенньй по формулам: $\phi_{n}\left(\xi_{i}\right)=\xi_{i}, \phi_{n}\left(\sigma_{i}\right)=\xi_{i}$. Его композиция с каноническим вложением $\nu_{n}$ группы $\Sigma_{n}$ в $B P_{n}$ равна тождественному гомоморфизму группы $\Sigma_{n}$. Эти гомоморфизмы порождают отображения классифицируюших пространств $B \nu_{n}$ и $B \phi_{n}$ такие, что их композищия $B \Sigma_{n} \rightarrow B B P_{n} \rightarrow B \Sigma_{n}$ равна тождественному отображению. Имеем также вложение $\kappa_{n}$ групшы кос в $B P_{n}$, которое порождает отображение классифишируюших пространств: $B B r_{n} \rightarrow B B P_{n}$. Композиция $\kappa_{n}$ и $\phi_{n}$ дает канонический эпиморфизм $\tau_{n}: B r_{n} \rightarrow \Sigma_{n}$.

Обозначим через $\mathscr{Z}$ строгую моноидальную (тензорную) категорию, объекты которой $\{\overline{0}, \overline{1}, \ldots\}$ соответствуют цельм числам от 0 до $\infty$, а морфизмы определены по формуле

$$
\operatorname{hom}(\bar{k}, \bar{l})= \begin{cases}\mathbb{Z}, & \text { если } k=l, \\ \varnothing, & \text { если } k \neq l .\end{cases}
$$


Произведение в $\mathscr{Z}$ определено на объектах как сумма неотрицательных чисел, а на морфизмах - как сумма целых чисел. Эта категория обладает симметрией, которая равна нулевому элементу для всех $\bar{m}$ и $\bar{n}$. Гомоморфизмы $\alpha_{n}$ индуцируют морфизм пермутативных категорий $A: \mathscr{B} \mathscr{P} \rightarrow \mathscr{Z}$ и отображения классифищируюших пространств

$$
B \alpha_{n}: B B P_{n} \rightarrow S^{1}
$$

Имеется также вложение групшы $\mathbb{Z}$ в $B r_{n}$

$$
\gamma: \mathbb{Z} \rightarrow B r_{n}
$$

отображающее образующую циклической группы на одну из образующих группы кос $\sigma_{i}$, скажем, $\sigma_{1}: \gamma(1)=\sigma_{1}$. Мы будем опускать индекс $n$ в обозначениях для групा и гомоморфизмов, когда $n=\infty$.

ТЕОрема 12.1. Существуют отображсения

$$
\psi: B \Sigma \times B B r \rightarrow B B P^{+}
$$

$$
\beta: \Omega B\left(\amalg_{n \geqslant 0} B B P_{n}\right) \rightarrow \Omega^{\infty} S^{\infty} \times S^{1}
$$

такие, что отображение $\psi$ становится петлевым отображсением после группового пополнения, а отображение $\beta$ есть бесконечнократное петлевое отображение, и оно расщепляется отображением

$$
\mathbb{Z} \times B \Sigma^{+} \times S^{1} \stackrel{\mathrm{Id} \times(B \gamma)^{+}}{\longrightarrow} \mathbb{Z} \times B \Sigma^{+} \times B B r^{+} \stackrel{\mathrm{Id} \times \psi^{+}}{\longrightarrow} \mathbb{Z} \times B B P^{+} .
$$

Если бесконечнократное пространство петель $Y$ является слоем отображсения $\beta$, то оно участвует в расщеплении

$$
\Omega B\left(\amalg_{n \geqslant 0} B B P_{n}\right) \simeq \Omega^{\infty} S^{\infty} \times S^{1} \times Y .
$$

То же самое верно и для редуцированной группь кос-перестановок с одним изменением: отображение

$$
\bar{\psi}: B \Sigma \times B B r \rightarrow B \overline{B P}^{+}
$$

становится двукратным петлевым отображсением после группового пополнения. 
ДокАЗАТЕльство. Морфизм $A$ порождает бесконечнократное петлевое отображение $\Omega B\left(\amalg_{n \geqslant 0} B B P_{n}\right) \rightarrow S^{1}$. Аналогично гомоморфизмы $\phi_{n}$ порождают морфизм пермутативных категорий $\mathscr{B} \mathscr{P} \rightarrow \Sigma$ и соответствующее бесконечнократное петлевое отображение

$$
\Omega B\left(\amalg_{n \geqslant 0} B B P_{n}\right) \rightarrow \Omega^{\infty} S^{\infty} .
$$

Обозначим через $\beta$ следуюшую композищию:

$$
\Omega B\left(\amalg_{n \geqslant 0} B B P_{n}\right) \stackrel{\text { diag }}{\longrightarrow} \Omega B\left(\amalg_{n \geqslant 0} B B P_{n}\right) \times \Omega B\left(\amalg_{n \geqslant 0} B B P_{n}\right) \longrightarrow \Omega^{\infty} S^{\infty} \times S^{1} .
$$

Гомоморфизмы $\nu$ и $\kappa$ порождают отображение произведений классифишируюших пространств:

$$
B \Sigma \times B B r \rightarrow B B P \times B B P .
$$

Пространство $B \mathrm{BP}^{+}$является $H$-пространством, следовательно, существует отображение

$$
\mu: B B P^{+} \times B B P^{+} \rightarrow B B P^{+} .
$$

Рассмотрим теперь композищию $f$ :

$$
B \Sigma \times B B r \stackrel{B \nu \times B \kappa}{\longrightarrow} B B P \times B B P \stackrel{q \times q}{\longrightarrow} B B P^{+} \times B B P^{+} \stackrel{\mu}{\longrightarrow} B B P^{+} .
$$

Из конструкции мы видим, что отображение

$$
B \Sigma \stackrel{\text { equiv }}{\longrightarrow} B \Sigma \times * \stackrel{\text { id } \times \text { incl }}{\longrightarrow} B \Sigma \times B B r \stackrel{f}{\longrightarrow} B B P^{+}
$$

гомотопно $q B \nu$, а отображение

$$
B B r \stackrel{\text { equiv }}{\longrightarrow} * \times B B r \stackrel{\text { incl } \times \text { id }}{\longrightarrow} B \Sigma \times B B r \stackrel{f}{\longrightarrow} B B P^{+}
$$

гомотопно $q B \kappa$. Используя тот факт, что $\mathbb{Z} \times B B P^{+}$есть пространство петель, мы немного изменим отображение $q B \kappa: B B r \rightarrow B B P^{+}$и определим отображение $g: B B r \rightarrow$ $B P^{+}$по формуле

$$
g(b)=(q B \tau(b))^{-1} \cdot q B \kappa(b), \quad b \in B B r .
$$

В этом случае композиция $B^{+} \phi \cdot g: B B r \rightarrow B \Sigma^{+}$гомотопна нулю. Обозначим через $\psi$ композицию

$$
B \Sigma \times B B r \stackrel{B \nu \times g}{\longrightarrow} B B P \times B B P \stackrel{q \times q}{\longrightarrow} B B P^{+} \times B B P^{+} \stackrel{\mu}{\longrightarrow} B B P^{+},
$$

через $\chi$ композицию

$$
B \Sigma \times S^{1} \stackrel{\mathrm{id} \times B \gamma}{\longrightarrow} B \Sigma \times B B r \stackrel{\psi}{\longrightarrow} B B P^{+} .
$$

Наконец, обозначим через $\bar{\psi}$ и $\bar{\chi}$ соответствуюшие отображения для редуцированной группы кос-перестановок. Таким образом, композиция

$$
B \Sigma \times B B r \stackrel{\psi}{\longrightarrow} B B P^{+} \stackrel{B^{+} \phi \times B^{+} \alpha}{\longrightarrow} B \Sigma^{+} \times S^{1}
$$


гомотопна произведению $q \times B \alpha$, а композищия

$$
B \Sigma \times S^{1} \stackrel{\chi}{\longrightarrow} B B P^{+} \stackrel{B^{+} \phi \times B^{+} \alpha}{\longrightarrow} B \Sigma^{+} \times S^{1}
$$

является каноническим отображением из пространства в его плюс-конструкцию. Это дает следующее расшепление:

$$
B \Sigma^{+} \times S^{1} \stackrel{\chi^{+}}{\longrightarrow} B B P^{+} \stackrel{B^{+} \phi \times B^{+} \alpha}{\longrightarrow} B \Sigma^{+} \times S^{1}
$$

Отображение $B \kappa$ включается в коммутативную диаграмму

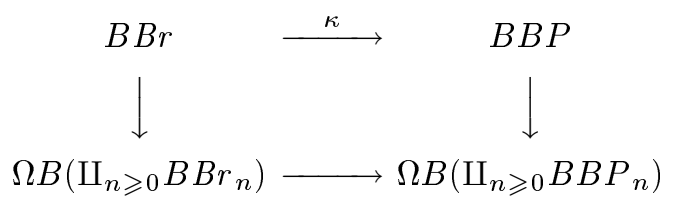

в которой нижняя стрелка является петлевым отображением.

Категория $\mathscr{B}$, образованная группами кос, является моноидальной категорией c заплетением (braided monoidal category - англ.), как это определено А. Жуаялем и Р. Стритом [83] (см. также дополнение). Следующая система элементов:

$$
\sigma_{m} \ldots \sigma_{1} \sigma_{m+1} \ldots \sigma_{2} \ldots \sigma_{n+m-1} \ldots \sigma_{n} \in B r_{m+n}
$$

определяет заплетение $c$ в $\mathscr{B}$. Графически такой элемент изображен на рис. Д.1 (см. дополнение). Образом заплетения $c$ при применении функтора $\overline{\mathscr{K}}: \mathscr{B} \rightarrow \overline{\mathscr{B} \mathscr{P}}$ является заплетение в категории $\overline{\mathscr{B} \mathscr{P}}$. Доказательство этого факта такое же, как и предложения 12.3 ниже. Отсюда и из теоремы Д.2 дополнения следует, что в нашем случае отображение классифицирующих пространств

$$
B \mathscr{B} \rightarrow B \overline{\mathscr{B} \mathscr{P}}
$$

индуцированное функтором $\overline{\mathscr{K}}$, становится двукратно петлевым после группового пополнения.

СлЕДСТВИЕ 12.1. В гомологиях с коэффициентами в $\mathbb{Z}$ эпиморфизм алгебр

$$
\beta_{*}: H_{*}(B P ; \mathbb{Z}) \rightarrow H_{*}(\Sigma \times \mathbb{Z} ; \mathbb{Z})
$$

расщепляется (как гомоморфизм абелевых групп) при помощи гомоморфизма:

$$
\chi_{*}: H_{*}(\Sigma \times \mathbb{Z} ; \mathbb{Z}) \rightarrow H_{*}(B P ; \mathbb{Z}) .
$$

Гомологии с коэффициентами в $\mathbb{Z} / p$ группы кос-перестановок из бесконечного числа нитей $H_{*}(B P, \mathbb{Z} / p)$ как $H_{*}(\Sigma ; \mathbb{Z} / p)$-модуль изоморфны

$$
H_{*}(\Sigma ; \mathbb{Z} / p) \otimes H_{*}(\mathbb{Z} ; \mathbb{Z} / p) \otimes H_{*}(Y ; \mathbb{Z} / p)
$$


То же самое верно и для гомологий редуцированной группь кос-перестановок $\overline{B P}$.

Гомоморфизм вложения $B P_{n} \rightarrow$ Aut $F_{n}$ порождает $E_{\infty}$-отображение классифицируюших пространств соответствуюших категорий $B \mathscr{B} \mathscr{P} \rightarrow B A u t$ и соответствующее отображение бесконечнократных пространств петель

$$
\Omega B\left(\amalg_{n \geqslant 0} B B P_{n}\right) \rightarrow \Omega B\left(\amalg_{n \geqslant 0} B \mathrm{Aut}_{n}\right) .
$$

Рассмотрим композищию

$$
B \Sigma^{+} \times \mathrm{BBr}^{+} \rightarrow B B P^{+} \rightarrow\left(B \mathrm{Aut}_{\infty}\right)^{+}
$$

и увидим, что в гомологиях по модулю $p$ элементы из $H_{*}(B P, \mathbb{Z} / p)$, которые являются образами элементов из $H_{*}(B r, \mathbb{Z} / p)$, и те, которые получаются из них применением операций Араки-Кудо-Даера-Лашофа [32], отображаются в образ $H_{*}(B \Sigma, \mathbb{Z} / p)$, лежащий в гомологиях группы $\mathrm{Aut}_{\infty}$.

Пусть $\pi_{i}^{S}, i=0,1, \ldots$, обозначают стабильные гомотопические групшы сфер. Бесконечнократные пространства петель $\mathbb{Z} \times B B P^{+}$и $\mathbb{Z} \times B \overline{B P}^{+}$определяют обобшенные теории гомологий, которые мы будем обозначать через $P B_{*}(\cdot)$ (чтобы не спутать с теорией Брауна-Петерсона) и $\overline{P B}_{*}(\cdot)$. Наши рассмотрения доказывают также следуюшее утверждение.

СлЕДСТВИЕ 12.2. Последовательность групп кос-перестановок определяет обобщенную мультипликативную теорию гомологий $P B_{*}(\cdot)$, группь коэффициентов которой $P B_{i}$ содержат группь $\pi_{i}^{S} \oplus \pi_{i}\left(S^{1}\right)$ в качестве прямого слагаемогов.

Рассмотрим теперь моноид Баеса-Бирман и группу кос с особенностями. Спаривания

$$
\begin{aligned}
& \mu_{m, n}: S B_{m} \times S B_{n} \rightarrow S B_{m+n}, \\
& \mu_{m, n}: S G_{m} \times S G_{n} \rightarrow S G_{m+n}
\end{aligned}
$$

определяются стандартньм образом по формулам

$$
\begin{gathered}
\mu_{m, n}\left(g_{i}^{\prime}\right)=g_{i}, \quad \mu_{m, n}\left(a_{i}^{\prime}\right)=a_{i} ; \\
g_{i}^{\prime}, a_{i}^{\prime} \in S B_{m}, S G_{m} ; \quad g_{i}, a_{i} \in S B_{m+n}, S G_{m+n} ; \\
\mu_{m, n}\left(g_{j}^{\prime \prime}\right)=g_{j+m}, \quad \mu_{m, n}\left(a_{j}^{\prime \prime}\right)=a_{j+m} ; \\
g_{i}^{\prime \prime}, a_{i}^{\prime \prime} \in S B_{n}, S G_{n} ; \quad g_{j+m}, a_{j+m} \in S B_{m+n}, S G_{m+n} .
\end{gathered}
$$

Эти спаривания согласованы с соответствующим спариванием для групш кос. Они определяют строгие моноидальные категории $\mathscr{S} \mathscr{B}$ и $\mathscr{S} \mathscr{G}$, а гомоморфизмы $j_{n}$ и канонические вложения $S B_{n} \rightarrow S G_{n}$ определяют функторы из строгой моноидальной категории $\mathscr{B}$, образованной группами кос

$$
\begin{gathered}
\mathscr{J}: \mathscr{B} \rightarrow \mathscr{S} \mathscr{B}, \\
\mathscr{B} \stackrel{\mathscr{J}}{\longrightarrow} \mathscr{S} \mathscr{B} \longrightarrow \mathscr{S} \mathscr{G},
\end{gathered}
$$

которые являются морфизмами моноидальных категорий. Спаривания $\mu$ коммутативны с точностью до сопряжения, так же как и для групп кос. Более точно, имеет место следующий факт. 
ПРЕДЛОЖЕНИЕ 12.3. Образ заплетения с в категории $\mathscr{B}$ при применении функтора $\mathscr{J}$ является заплетением в категории $\mathscr{S} \mathscr{B}$, так что последняя становится категорией с заплетением, а функтор $\mathscr{J}$ - морфизмом категорий с заплетением. Аналогично, категория $\mathscr{S} \mathscr{G}$ является категорией с заплетением, а соответствующие функторь - морфизмами категорий с заплетением.

ДокаЗАТЕльство. Мы доказьваем утверждение для категории $\mathscr{S} \mathscr{B}$. Доказательство для $\mathscr{S} \mathscr{G}$ то же самое. По определению естественность заплетения $\mathscr{J}(c)$ (которое мы будем для простоты обозначать тем же символом $c$ ) означает, что справедливо следующее равенство:

$$
c_{\bar{m}, \bar{n}} \cdot \mu\left(b_{m}^{\prime}, b_{n}^{\prime \prime}\right)=\mu\left(b_{n}^{\prime \prime}, b_{m}^{\prime}\right) \cdot c_{\bar{m}, \bar{n}} .
$$

Это эквивалентно соотношению

$$
c_{\bar{m}, \bar{n}} \cdot \mu\left(b_{m}^{\prime}, b_{n}^{\prime \prime}\right) \cdot c_{\bar{m}, \bar{n}}^{-1}=\mu\left(b_{n}^{\prime \prime}, b_{m}^{\prime}\right),
$$

которое означает, что сопряжение элементом $c_{\bar{m}}, \bar{n}$ преобразует элементы из $S B_{m} \times S B_{n}$, канонически вложенного в $S B_{m+n}$, в соответствуюшие элементы из $S B_{n} \times S B_{m}$. Элементы $c_{\bar{m}, \bar{n}}$ определяют заплетение для категории $\mathscr{B}$ так, что для проверки естественности $c$ в $\mathscr{S} \mathscr{B}$ осталось проверить ее на образующих $a_{i}$, $1 \leqslant i \leqslant m-1, m \leqslant i \leqslant m+n$. Рассмотрим соответствующее сопряжение:

$$
g_{m} \ldots g_{1} g_{m+1} \ldots g_{2} \ldots g_{n+m-1} \ldots g_{n} a_{i} g_{n}^{-1} \ldots g_{n+m-1}^{-1} \ldots g_{2}^{-1} g_{m+1}^{-1} g_{1}^{-1} \ldots g_{m}^{-1} .
$$

Если $i>n$, мы двигаем $a_{i}$ влево, используя соотношение $a_{i} g_{i+1} g_{i}=g_{i+1} g_{i} a_{i+1}$. Имеем:

$$
\begin{aligned}
g_{m} \ldots & g_{1} g_{m+1} \ldots g_{2} \ldots g_{n+m-1} \ldots g_{n} a_{i} g_{n}^{-1} \ldots g_{n+m-1}^{-1} \ldots g_{2}^{-1} g_{m+1}^{-1} g_{1}^{-1} \ldots g_{m}^{-1} \\
= & g_{m} \ldots g_{1} g_{m+1} \ldots g_{2} \ldots g_{n+m-1} \\
& \ldots g_{i-1} a_{i} g_{i-1}^{-1} \ldots g_{n+m-1}^{-1} \ldots g_{2}^{-1} g_{m+1}^{-1} g_{1}^{-1} \ldots g_{m}^{-1} \\
= & g_{m} \ldots g_{1} g_{m+1} \ldots g_{2} \ldots g_{n+m-1} \\
& \ldots g_{i+1} a_{i-1} g_{i} g_{i-1} g_{i-1}^{-1} \ldots g_{n+m-1}^{-1} \ldots g_{2}^{-1} g_{m+1}^{-1} g_{1}^{-1} \ldots g_{m}^{-1}=\ldots=a_{i-n}
\end{aligned}
$$

Если $i<n$, мы двигаем $a_{i}$ влево, используя соотношение $a_{i+1} g_{i} g_{i+1}=g_{i} g_{i+} a_{i}$. Имеем:

$$
\begin{aligned}
g_{m} \ldots & g_{1} g_{m+1} \ldots g_{2} \ldots g_{n+m-1} \ldots g_{n} a_{i} g_{n}^{-1} \ldots g_{n+m-1}^{-1} \ldots g_{2}^{-1} g_{m+1}^{-1} g_{1}^{-1} \ldots g_{m}^{-1} \\
= & g_{m} \ldots g_{1} g_{m+1} \ldots g_{2} \ldots g_{i} g_{n+m-1} \\
& \ldots g_{i+2} g_{i+1} a_{i} g_{i+1}^{-1} \ldots g_{n+m-1}^{-1} \ldots g_{2}^{-1} g_{m+1}^{-1} g_{1}^{-1} \ldots g_{m}^{-1} \\
= & g_{m} \ldots g_{1} g_{m+1} \ldots g_{2} \ldots g_{i} g_{n+m-1} \\
& \ldots g_{i+2} g_{i}^{-1} g_{i} g_{i+1} a_{i} g_{i+1}^{-1} \ldots g_{n+m-1}^{-1} g_{i}^{-1} \ldots g_{2}^{-1} g_{m+1}^{-1} g_{1}^{-1} \ldots g_{m}^{-1} \\
= & g_{m} \ldots g_{1} g_{m+1} \ldots g_{2} \ldots g_{i+1} g_{n+m-1} \\
& \ldots g_{i+2} a_{i+1} g_{i} g_{i+1} g_{i+1}^{-1} \ldots g_{n+m-1}^{-1} g_{i}^{-1} \ldots g_{2}^{-1} g_{m+1}^{-1} g_{1}^{-1} \ldots g_{m}^{-1} \\
= & g_{m} \ldots g_{1} g_{m+1} \ldots g_{2} \ldots g_{i+1} g_{n+m-1} \\
& \ldots g_{i+2} a_{i+1} g_{i+2}^{-1} \ldots g_{n+m-1}^{-1} g_{i+1}^{-1} \ldots g_{2}^{-1} g_{m+1}^{-1} g_{1}^{-1} \ldots g_{m}^{-1}=\ldots=a_{i+m} .
\end{aligned}
$$


Условия когерентности выполнены очевидным образом. Для условия В1 из [83] имеем тождество:

$$
\begin{aligned}
g_{m} \ldots g_{1} g_{m+1} \ldots g_{2} \ldots g_{n+m-1} \ldots g_{n} & \\
& \quad \times g_{m+n} \ldots g_{n+1} g_{m+n+1} \ldots g_{n+2} \ldots g_{n+m+q-1} \ldots g_{n+q} \\
= & g_{m} \ldots g_{1} g_{m+1} \ldots g_{2} \ldots g_{n+m+q-1} \ldots g_{n+q} .
\end{aligned}
$$

Для В2 очевидно, что

$$
\begin{aligned}
g_{m+n} & \ldots g_{m+1} g_{m+n+1} \ldots g_{m+2} \ldots g_{n+m+q-1} \ldots g_{m+q} \\
& \times g_{m} \ldots g_{1} g_{m+1} \ldots g_{2} \ldots g_{m+q-1} \ldots g_{q} \\
= & g_{m+n} \ldots g_{1} g_{m+n+1} \ldots g_{2} \ldots g_{m+n+q-1} \ldots g_{q} .
\end{aligned}
$$

Пусть $B S B$ и $B S G$ обозначают классифицирующие пространства предельного моноида Баеса-Бирман и соответствуюшей группы кос с особенностями. Спаривания $\mu_{m, n}$ определяют, как обычно, структуру моноида на несвязной сумме классифицирующих пространств $S B_{n}$ и $S G_{n}$ :

$$
\amalg_{n \geqslant 0} B S B_{n}, \quad \amalg_{n \geqslant 0} B S G_{n} .
$$

ПРЕДЛОЖЕНИЕ 12.4. Канонические отображения

$$
\begin{aligned}
& B S B \rightarrow \Omega B\left(\amalg_{n \geqslant 0} B S B_{n}\right), \\
& B S G \rightarrow \Omega B\left(\amalg_{n \geqslant 0} B S G_{n}\right)
\end{aligned}
$$

индуцируют изоморфизмы в гомологиях

$$
\begin{aligned}
& H_{*}(B S B ; A) \rightarrow H_{*}\left(\left(\Omega B\left(\amalg_{n \geqslant 0} B S B_{n}\right)\right)_{0} ; A\right), \\
& H_{*}(B S G ; A) \rightarrow H_{*}\left(\left(\Omega B\left(\amalg_{n \geqslant 0} B S G_{n}\right)\right)_{0} ; A\right)
\end{aligned}
$$

с любими (постоянными) коэффициентами. Следовательно, имеют место изоморфизмь

$$
\begin{aligned}
& B S B^{+} \cong\left(\Omega B\left(\amalg_{n \geqslant 0} B S B_{n}\right)\right)_{0}, \\
& B S G^{+} \cong\left(\Omega B\left(\amalg_{n \geqslant 0} B S G_{n}\right)\right)_{0} .
\end{aligned}
$$

Доказательство этого факта такое же, как доказательство теоремы 3.2.1 и следствия 3.2.2 в [66], или (что по сушеству то же самое) основывается непосредственно на результатах работы [67]. Заплетение $c$ дает необходимую гомотопическую коммутативность для $H$-пространств $\amalg_{n \geqslant 0} B S B_{n}$ и $\amalg_{n \geqslant 0} B S G_{n}$.

Сравнивая соотношения, определяюшие моноид Баеса-Бирман и редуцированную группу кос-перестановок, мы видим, что можно определить очевидньй гомоморфизм $l_{n}: S B_{n} \rightarrow \overline{B P}_{n}$ по формулам: $l_{n}\left(g_{i}\right)=\sigma_{i}, l_{n}\left(a_{i}\right)=\xi_{i}$. Эти же формулы определяют гомоморфизм $S G_{n} \rightarrow \overline{B P}_{n}$. Композиция $l_{n} \circ j_{n}$ равна каноническому гомоморфизму $\kappa_{n}: B r_{n} \rightarrow \overline{B P}_{n}$. Гомоморфизмы $l_{n}, n=0,1 \ldots$, определяют морфизм моноидальных категорий $\mathscr{L}: \mathscr{S} \mathscr{B} \rightarrow \mathscr{S} \mathscr{G} \rightarrow \overline{\mathscr{B} \mathscr{P}}$ такой, что образ заплетения $c$ является заплетением в категории $\overline{\mathscr{B} \mathscr{P}}$. 
ТЕОРема 12.2. Гомоморфизмы $j_{n} u l_{n}$ индуцируют морфизмы категорий $c$ заплетением

$$
\mathscr{B} \stackrel{\mathscr{J}}{\longrightarrow} \mathscr{S} \mathscr{B} \longrightarrow \mathscr{S} \mathscr{G} \stackrel{\mathscr{L}}{\longrightarrow} \overline{\mathscr{B} \mathscr{P}}
$$

и соответствующие двукратно петлевие отображения

$$
\Omega^{2} S^{2} \longrightarrow \Omega B\left(\amalg_{n \geqslant 0} B S B_{n}\right) \longrightarrow \Omega B\left(\amalg_{n \geqslant 0} B S G_{n}\right) \longrightarrow \Omega B\left(\amalg_{n \geqslant 0} B \overline{B P}_{n}\right) .
$$

Доказательство следует из того факта, что классифищирующее пространство категории с заплетением является двукратным пространством петель после группового пополнения.

ПРЕДЛОЖЕНИЕ 12.5. Абелеанизаџия группь $S G_{n}$ равна $\mathbb{Z} \oplus \mathbb{Z}$ :

$$
S G_{n} /\left[S G_{n}, S G_{n}\right] \cong \mathbb{Z} \oplus \mathbb{Z}
$$

ДокаЗАТЕльство.Добавим следуюшие соотношения к соотношениям группы кос с особенностями $S G_{n}: g_{i}=g_{j}$ для всех $i$ и $j, a_{i}=a_{j}$ для всех $i$ и $j$, и получим эпиморфизм $a b_{S B}: S B_{n} \rightarrow \mathbb{Z} \oplus \mathbb{Z}$. Обозначим через $g$ и $a$ образующие $\mathbb{Z} \oplus \mathbb{Z}$, являющиеся образами соответствуюших образующих $S G_{n}$. Предположим, что имеется гомоморфизм в некоторую абелеву группу $A, f: S G_{n} \rightarrow A$. Из соотношений группы кос с особенностями $S G_{n}$ следует, что $f\left(g_{i}\right)=f\left(g_{j}\right)$ и $f\left(a_{i}\right)=f\left(a_{j}\right)$ для всех $i$ и $j$. Следовательно, гомоморфизм $f^{\prime}: \mathbb{Z} \oplus \mathbb{Z} \rightarrow A$ однозначно определяется по формулам $f^{\prime}(g)=f\left(g_{i}\right), f^{\prime}(a)=f\left(a_{i}\right)$. Это означает, что эпиморфизм $a b_{S B}$ является гомоморфизмом абелеанизации $S G_{n}$.

Сушествуют гомоморфизмы, являюшиеся правыми обратными к гомоморфизму $j_{n}: B r_{n} \rightarrow S G_{n}$. Определим один из них $h_{n}: S G_{n} \rightarrow B r_{n}$ по формулам $h_{n}\left(g_{i}\right)=\sigma_{i}$, $h_{n}\left(a_{i}\right)=e$, а второй $h_{n}^{\prime}: S G_{n} \rightarrow B r_{n}$, исходя из следующего действия на образуюшие: $h_{n}^{\prime}\left(g_{i}\right)=\sigma_{i}, h_{n}^{\prime}\left(a_{i}\right)=\sigma_{i}$. Пусть $\operatorname{deg}_{n}$ есть гомоморфизм $S G_{n} \rightarrow \mathbb{Z}$, сопоставляющий каждому элементу групшы $S G_{n}$ сумму степеней образуюших $a_{i}$, входящих в запись этого элемента. Определим заплетение

$$
c_{\bar{m}, \bar{n}}: \overline{m+n} \rightarrow \overline{m+n}
$$

в категории $\mathscr{Z}: c_{\bar{m}, \bar{n}}=m \cdot n$. Тогда гомоморфизмы $\operatorname{deg}_{n}$ порождают функтор категорий с заплетением $\mathscr{S} \mathscr{G} \rightarrow \mathscr{Z}$ и соответствующее двукратно петлевое отображение

$$
\Omega B\left(\amalg_{n \geqslant 0} B S G_{n}\right) \rightarrow S^{1} .
$$

Аналогично гомоморфизмы $h_{n}$ порождают функтор категорий с заплетением $\mathscr{S} \mathscr{G} \rightarrow$ $\mathscr{B}$ и соответствующее двукратно петлевое отображение

$$
\Omega B\left(\amalg_{n \geqslant 0} B S G_{n}\right) \rightarrow \Omega^{2} S^{2} .
$$

Обозначим через $\lambda$ композицию

$$
\Omega B\left(\amalg_{n \geqslant 0} B S G_{n}\right) \stackrel{\operatorname{diag}}{\longrightarrow} \Omega B\left(\amalg_{n \geqslant 0} B S G_{n}\right) \times \Omega B\left(\amalg_{n \geqslant 0} B S G_{n}\right) \longrightarrow S^{1} \times \Omega^{2} S^{2} .
$$

Пусть $W$ есть двукратное пространство петель, являюшееся слоем отображения $\lambda$. Определим гомоморфизм $\mathbb{Z} \rightarrow S G_{n}$ как вложение циклической группы в качестве подгруппы, порожденной элементом $a_{1}$. Тогда композиция $\mathbb{Z} \longrightarrow S G_{n} \stackrel{\operatorname{deg}_{n}}{\longrightarrow} \mathbb{Z}$ равна тождественному гомоморфизму. Так же, как в доказательстве теоремы 12.1 , мы строим отображение $\psi_{S}: S^{1} \times B B r \rightarrow B S G^{+}$. 
Теорема 12.3. Двукратно петлевое отображение

$$
\lambda: \Omega B\left(\amalg_{n \geqslant 0} B S G_{n}\right) \rightarrow S^{1} \times \Omega^{2} S^{2}
$$

расщепляется петлевым отображением

$$
1 \times \psi_{S}^{+}: \mathbb{Z} \times S^{1} \times B B r^{+} \rightarrow \mathbb{Z} \times B S G^{+} .
$$

Следовательно, имеется эквивалентность

$$
\mathbb{Z} \times B S G^{+} \simeq S^{1} \times \Omega^{2} S^{2} \times W
$$

Доказательство аналогично доказательству соответствующего утверждения теоремы 12.1.

СЛЕДСТВИЕ 12.3. В гомологиях с чельми коэффициентами эпиморфизм ал$2 e 6 p$

$$
\lambda_{*}: H_{*}(S G ; \mathbb{Z}) \rightarrow H_{*}(\mathbb{Z} \times B r ; \mathbb{Z})
$$

расщепляется при помощи мономорфизма:

$$
\psi_{S_{*}}: H_{*}(\mathbb{Z} \times B r ; \mathbb{Z}) \rightarrow H_{*}(S G ; \mathbb{Z}) .
$$

Гомологии с коэффициентами в $\mathbb{Z} / p$ группы кос с особенностями из бесконечного числа нитей $H_{*}(S G ; \mathbb{Z} / p)$ изоморфньи $H_{*}(\mathbb{Z} ; \mathbb{Z} / p) \otimes H_{*}(B r ; \mathbb{Z} / p) \otimes H_{*}(W ; \mathbb{Z} / p)$ как алгебрыл.

Результаты этого параграфа были получены в [84].

\section{$\S$ 13. Косы и спектры Тома}

Для изучения спектров Тома для кос нам потребуются некоторые сведения из теории гомотопий, связанные с теоремой М. Маховальда [39], [40]. Предположим в этом параграфе (до теоремы 13.6), что пространства и спектры локализованы вне 2.

Предельная ортогональная группа $O$ и ее классифишируюшее пространство $B O$ являются бесконечнократными пространствами петель, следовательно, определены итерированные классифищируюшие пространства $B^{k} O$.

Пусть $X$ есть $C W$-комплекс, заданньй вместе с отображениями $f: S^{n+1} \rightarrow X$ и $g: X \rightarrow B^{n+1} O$ такими, что вьполнено следуюшее условие. Рассмотрим композицию

$$
g f: S^{n+1} \rightarrow X \rightarrow B^{n+1} O
$$

и применим функтор $n$-кратных петель к ней:

$$
\Omega^{n} g f: \Omega^{n} S^{n+1} \rightarrow \Omega^{n} B^{n+1} O \simeq B O .
$$

Пусть $S^{1} \rightarrow \Omega^{n} S^{n+1}$ есть вложение, являющееся $n$-м сопряженным к тождественному отображению $S^{n+1} \rightarrow S^{n+1}$. Условие состоит в том, что композиция

$$
S^{1} \rightarrow \Omega^{n} S^{n+1} \rightarrow B O
$$

нетривиальна. Отображение $\Omega^{n} g: \Omega^{n} X \rightarrow B O$ порождает спектр Тома $M(X, n)$. 
Пусть теперь $X=S^{3}, n=2$ и отображение $g: S^{3} \rightarrow B^{3} O$ нетривиально. Оно индуцирует отображение $\Omega^{2} g: \Omega^{2} S^{3} \rightarrow B O$, которое в свою очередь порождает отображение

$$
\Omega^{2} g: \Omega^{2} S^{2} \rightarrow \mathbb{Z} \times B O .
$$

Теорема 13.1 (М. Маховальд). Спектр Тома $M\left(S^{3}, 2\right)$ әквивалентен спектру Эйленберга-Маклейна $H \mathbb{Z} / 2$ :

$$
M\left(S^{3}, 2\right) \simeq H \mathbb{Z} / 2 .
$$

ДокАЗАТЕЛЬСТВо. Имеется стандартное отображение из алгебры Стинрода по модулю $2 \mathscr{A}$ в $H^{*}\left(M\left(S^{3}, 2\right) ; \mathbb{Z} / 2\right)$, задаваемое сопоставлением $S q^{I}$ элемента $S q^{I}(U)$, где $U$ - класс Тома. Для доказательства теоремы достаточно установить, что это отображение есть изоморфизм.

Заметим:

1) Ряды Эйлера-Пуанкаре для $H^{*}\left(\Omega^{2} S^{3} ; \mathbb{Z} / 2\right)$ и $\mathscr{A}$ совпадают. Следовательно, достаточно увидеть, что рассматриваемое отображение есть мономорфизм, так как каждый ряд "конечного типа".

2) Рассматриваемое отображение есть морфизм коалгебр, и, следовательно, достаточно увидеть, что оно инъективно на модуле примитивных.

3) Модуль примитивных для $\mathscr{A}$ задается милноровскими $Q_{i}$, и имеется не больше одного из них в каждой размерности. Следовательно, достаточно проверить, что каждый $Q_{i}$ имеет ненулевой образ.

4) Обозначим через $w_{i} i$-й класс Штифеля-Уитни, а $x_{2^{k}-1}$ есть единственный примитивный элемент из $H_{*}\left(\Omega^{2} S^{3} ; \mathbb{Z} / 2\right)$ в размерности $2^{k}-1$. Из работы $\Phi$. П. Петерсона и Х. Тоды [85] следует, что $Q_{k}(U)=\left(w_{2^{k}-1}+\right.$ разложимые $) U$ в $H^{*}(M O ; \mathbb{Z} / 2)$.

5) Пусть $\langle$, $\rangle$ обозначает обычное кронекеровское произведение, которое вычисляет значение когомологического класса на гомологическом той же размерности. Ниже мы проверим, что

$$
\left\langle g^{*}\left(w_{2^{k}-1}\right), x_{2^{k}-1}\right\rangle=1
$$

и

$$
\left\langle g^{*} \text { (разложимые) }, x_{2^{k}-1}\right\rangle=0 .
$$

Тогда $Q_{k}(U)$ не равен нулю в $H^{*}\left(M\left(S^{3}, 2\right) ; \mathbb{Z} / 2\right)$ и теорема будет доказана.

6) Поскольку $x_{2^{k}-1}$ примитивен, $\left\langle g^{*}\right.$ (разложимые), $\left.x_{2^{k}-1}\right\rangle$ равно нулю. Отсюда следует формула (13.3).

7) Чтобы проверить (13.2), положим $S q^{I}=S q^{1} S q^{2} S q^{4} \ldots S q^{2^{k-2}}$. Следовательно,

$$
S q^{I} w_{2^{k-1}}=w_{2^{k-1}}+\text { разложимые }
$$

по формуле Ву. Значит,

$$
\begin{aligned}
\left\langle\left(\Omega^{2} g\right)^{*}\left(w_{2^{k}-1}\right), x_{2^{k}-1}\right\rangle & =\left\langle\left(\Omega^{2} g\right)^{*} S q^{I} w_{2^{k-1}}, x_{2^{k}-1}\right\rangle=\left\langle S q^{I} w_{2^{k-1}},\left(\Omega^{2} g\right)_{*} x_{2^{k}-1}\right\rangle \\
& =\left\langle w_{2^{k-1}},\left(\Omega^{2} g\right)_{*} S q_{*}^{2^{k-2}} \ldots S q_{*}^{2} S q_{*}^{1} x_{2^{k}-1}\right\rangle \\
& =\left\langle w_{2^{k-1}},\left(\Omega^{2} g\right)_{*} x_{1}^{2^{k-1}}\right\rangle=1,
\end{aligned}
$$

так как $w_{2^{k-1}}$ двойствен примитивному классу в $H_{*}(B O ; \mathbb{Z} / 2)$, заданному $2^{k-1}$-й степенњю одномерного класса. 
ЗАмЕчАния. 1) Это доказательство никак не использует операции Араки-КудоДаера-Лашофа. Для него только требуется наличие некоторого петлевого отображения из $\Omega^{2} S^{3}$ в $B O$, ненулевого на первой группе гомологий.

2) Это доказательство приведено в [86] и было намечено в [31].

Теорема 13.2. Спектр Тома $M(X, n), n \geqslant 2$, есть букет надстроек над спектром Эйленберга-Маклейна $H \mathbb{Z} / 2$.

Доказательство следует из теоремы М. Маховальда.

Перед тем как рассматривать спектры Тома для кос, дадим некоторые общие определения и конструкции.

Предположим, что заданы последовательность конечно представленных моноидов $H_{n}$ для $n=0,1,2, \ldots$ и гомоморфизмы $g_{n}: H_{n} \rightarrow H_{n+1}$. Обозначим через $g_{n+m}^{n}$ композицию $g_{n+m-1} \ldots g_{n}, g_{n+m}^{n}: H_{n} \rightarrow H_{n+m}$, а через $H$ прямой предел системы $\left\{H_{n}, g_{n}\right\}$. Предположим также, что для всех $m$ и $n$ сушествуют гомоморфизмы

$$
\nu_{m, n}: B r_{m} \times H_{n} \rightarrow H_{m+n}
$$

такие, что $\nu_{0, n}(e, x)=\operatorname{Id}(x), x \in H_{n}$ и $е$ есть единица группы $B r_{0}=\{e\}$, и диаграммы

$$
\begin{aligned}
& B r_{l} \times B r_{m} \times H_{n} \stackrel{\mathrm{Id} \times \nu_{m, n}}{\longrightarrow} B r_{l} \times H_{m+n} \\
& \downarrow \mu_{l, m} \times \mathrm{Id} \quad \downarrow \nu_{l, m+n} \\
& B r_{l+m} \times H_{n} \stackrel{\nu_{l+m, n}}{\longrightarrow} H_{l+m+n} \\
& B r_{l} \times H_{m} \stackrel{i_{l+k}^{l} \times g_{m+n}^{m}}{\longrightarrow} B r_{l+k} \times H_{m+n} \\
& \downarrow \nu_{l, m} \quad \downarrow \nu_{l+k, m+n} \\
& H_{l+m} \stackrel{g_{l+k+m+n}^{l+m}}{\longrightarrow} H_{l+k+m+n}
\end{aligned}
$$

коммутативны с точностью до сопряжения обратимыми элементами моноидов.

Мы называем такую систему моноидов модульной над косами системой моноидов. Если группы кос заменены системой симметрических групп в приведенном выше условии, то такая система моноидов будет называться симметрически модульной системой моноидов.

Предположим, что система моноидов $\left\{H_{n}, g_{n}\right\}$ такова, что $H_{0}$ состоит из одного элемента $H_{0}=\{e\}$. Тогда $\nu_{m, 0}$ есть гомоморфизм из $B r_{m}$ в $H_{m}$. Через канонические гомоморфизмыs $\tau_{m}: B r_{m} \rightarrow \Sigma_{m}$ каждая симметрически модульная система моноидов наделяется структурой модульной над косами системы. Предположим теперь, что система моноидов $\left\{H_{n}, g_{n}\right\}$ такова, что для всех $m$ и $n$ сушествуют гомоморфизмы

$$
\mu_{m, n}^{H}: H_{m} \times H_{n} \rightarrow H_{m+n}
$$


такие, что $\mu_{e, n}^{H}(e, x)=\operatorname{Id}(x)$ и $\mu_{m, n}^{H}(x, e)=\operatorname{Id}(x)$, где $x \in H_{n}$ и $e-$ единица моноида $H_{0}$, а диаграмма

$$
\begin{aligned}
& H_{m} \times H_{n} \stackrel{g_{m+k}^{m} \times g_{n+l}^{n}}{\longrightarrow} H_{m+k} \times H_{n+l} \\
& \downarrow \mu_{l, m}^{H} \quad \downarrow \mu_{m+k, n+l}^{H} \\
& H_{l+m} \stackrel{g_{m+k+n+l}^{l+m}}{\longrightarrow} H_{m+k+n+l}
\end{aligned}
$$

и аналог диаграммы (2.8) коммутативны с точностью до сопряжения обратимыми элементами моноидов.

Мы назьваем такую систему моноидов моноидальной системой моноидов. Если моноидальная и модульная над косами структуры системы моноидов согласованы очевидным образом, то мы говорим, что $\left\{H_{n}\right\}$ есть алгебра над косами.

Морфизмы моноидальных систем моноидов определяются обычным образом. Групшы кос и симметрические групшы образуют моноидальные системы групा $\left\{B r_{n}, i_{n}\right\}$ и $\left\{\Sigma_{n}, i_{n}\right\}$ стандартным образом. Если имеется моноидальная система моноидов $\left\{H_{n}, g_{n}\right\}$ и морфизмы моноидальных систем

$$
\phi:\left\{B r_{n}, i_{n}\right\} \rightarrow\left\{H_{n}, g_{n}\right\},
$$

то система $\left\{H_{n}, g_{n}\right\}$ становится модульной над косами системой моноидов.

Пусть $K$ есть ассоциативное кольцо с единицей 1 , а $s_{m}$ - каноническое представление из симметрической групшы $\Sigma_{m}$ в $G L_{m} K$. Стандартные вложения $G L_{m} K$ и $G L_{n} K$ в качестве диагональных блоков в $G L_{m+n} K$ определяют моноидальную структуру для системы групп $G L_{n} K$. Морфизмы $s_{m}$ и $s_{m} \tau_{m}$ определяют структуры симметрически модульной системы и модульной над косами системы групा $G L_{n} K$. Обозначим эту модульную над косами структуру через $\psi$. Предположим теперь, что имеется модульная над косами система моноидов $\left\{H_{n}, g_{n}\right\}$ и модульньй над косами морфизм $\rho$ из $\left\{H_{n}, g_{n}\right\}$ в $\left\{G L_{n} K\right\}$. Это означает, что имеется система гомоморфизмов (представлений)

$$
\rho_{n}: H_{n} \rightarrow G L_{n} K
$$

Предположим, что диаграммы

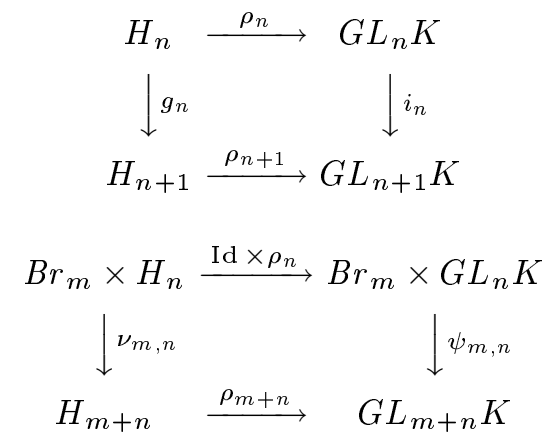

коммутативны с точностью до сопряжения. 
Мы говорим в этой ситуации, что система представлений $\rho_{n}$ согласована с модульной над косами структурой системы моноидов $H_{n}$. Аналогично мы определяем, что система представлений согласована с симметрически модульной структурой системы моноидов.

Сушествует большое количество примеров структур, описанных выше. Некоторые из них уже обсуждались: полные линейные группы $\left\{G L_{n} K\right\}$ образуют структуру алгебры над симметрической группой, сами симметрические группы $\left\{\Sigma_{n}\right\}$ образуют структуру алгебры над косами. Любая система конечных групп Кокстера $\left\{W_{n}\right\}$, действуюших на $\mathbb{R}^{n}$, образует симметрически модульную структуру, а соответствующая система обобщенных групп кос $\left\{B r\left(W_{n}\right)\right\}$ образует модуль над косами. Пусть $G$ есть группа, а $\gamma$-гомоморфизм из $G$ в $\mathbb{Z} / 2 ; \gamma: G \rightarrow \mathbb{Z} / 2$. Рассмотрим сплетение $\Sigma_{n} \int G$. Гомоморфизм $\gamma$ индуцирует гомоморфизм $\gamma_{n}: \Sigma_{n} \int G \rightarrow \Sigma_{n} \int \mathbb{Z} / 2$. Последнее сплетение $\Sigma_{n} \int \mathbb{Z} / 2$ изоморфно группе Кокстера $C_{n}$ и, следовательно, вложено в ортогональную группу $O_{n}$. Таким образом, имеется композиция

$$
\Sigma_{n} \int G \rightarrow \Sigma_{n} \int \mathbb{Z} / 2 \rightarrow O_{n} \rightarrow G L_{n} \mathbb{R}
$$

дающая необходимое представление $\Sigma_{n} \int G$. Имеются канонические спаривания

$$
\begin{aligned}
& \left(\Sigma_{k} \times \Sigma_{n}\right) \int G \rightarrow \Sigma_{n+k} \int G, \\
& \Sigma_{k} \times\left(\Sigma_{n} \int G\right) \rightarrow \Sigma_{n+k} \int G,
\end{aligned}
$$

снабжающие $\left\{\Sigma_{n} \int G\right\}$ структурой алгебры над симметрической группой, а представление (13.5) согласовано с этой структурой. Аналогично можно рассматривать сплетение группы $G$ с группами кос.

Другие примеры описанных вьшше структур дают группы Aut $F_{n}, n=1,2, \ldots$, aвтоморфизмов свободных групп $F_{n}$ и их подгрупшы. Канонические вложения $(2.3)$ порождают структуру моноидальной системы групп. Стандартные вложения $t$ и $r$ симметрической группы и группы кос в Aut $F_{n}$ описаны в $\S 2$. Композиция морфизмов $t$ и $\tau$ дает еще один морфизм моноидальных систем групп

$$
\tau t:\left\{B r_{n}\right\} \rightarrow\left\{\text { Aut } F_{n}\right\}
$$

Гомоморфизм $t$ определяет структуру алгебры над симметрической группой для системы Aut $F_{n}$, а гомоморфизмы $r$ и $\tau t$ определяют две различные структуры алгебры над косами для системы групп Aut $F_{n}$. Абелеанизация свободной групшы $F_{n}$ есть свободная абелева группа ранга $n$. Это определяет эпиморфизм

$$
\rho_{n}: \text { Aut } F_{n} \rightarrow G L_{n} \mathbb{Z}
$$

Система представлений $\rho_{n}$ согласована со структурой алгебры над симметрической группой и двумя структурами алгебры над косами для системы групп Aut $F_{n}$.

Система моноидов $H_{n}<$ Aut $F_{n}, H_{n}<H_{n+1}$, содержаших симметрические группы:

$$
\Sigma_{n}<H_{n}<\operatorname{Aut} F_{n}
$$


или группы кос:

$$
B r_{n}<H_{n}<\text { Aut } F_{n}
$$

такая, что композиции

$$
\begin{aligned}
\Sigma_{n} & \rightarrow H_{n} \rightarrow \operatorname{Aut} F_{n} \rightarrow G L_{n} \mathbb{Z}, \\
B r_{n} & \rightarrow H_{n} \rightarrow \text { Aut } F_{n} \rightarrow G L_{n} \mathbb{Z}
\end{aligned}
$$

являются каноническими представлениями, является симметрически модульной системой или модульной над косами системой моноидов. Более конкретным примером этой ситуации является группа кос-перестановок $B P_{n}$ из $\S 5$. Как и в случае групп Aut $F_{n}$, гомоморфизмы $t$ индуцируют структуру алгебры над симметрической группой. Гомоморфизмы $r$ и $\tau t$ определяют две различные структуры алгебры над косами для системы групп $B P_{n}$. Система представлений, возникающая из Aut $F_{n}$, согласована со структурами алгебры над симметрической группой и алгебры над косами на $B P_{n}$.

Редуцированная группа кос-перестановок $\overline{B P}_{m}$ из $\S 5$ не является подгруппой Aut $F_{m}$. Однако, используя представление этой группы, мы можем определить структуру алгебры над симметрической группой и две различные структуры алгебры над косами для системы групп $\bar{B} P_{m}$. Представления Aut $F_{m} \rightarrow G L_{m} \mathbb{Z}$, $m=0,1, \ldots$, отображают элементы $\xi_{i}$ и $\sigma_{i}$ в одну и ту же матрицу. Следовательно, представления

$$
\overline{B P}_{m} \rightarrow G L_{m}(\mathbb{Z}), \quad m=0,1, \ldots,
$$

корректно определены и согласованы со структурами алгебры над симметрической группой и алгебры над косами на $\overline{B P}_{n}$.

Пусть $\operatorname{Aut}\left(\vee^{k} S^{n}\right)$ есть моноид пунктированных самоэквивалентностей букета $n$-мерных сфер $\vee^{k} S^{n}$. Пусть $V_{k}$ определено следуюшим образом:

$$
V_{k}=\underset{n}{\lim } \operatorname{Aut}\left(\vee^{k} S^{n}\right)
$$

Спаривание (13.4) задается для системы $\pi_{0}\left(V_{k}\right)$ очевидньм образом. Отображения

$$
\operatorname{Aut}\left(\bigvee^{k} S^{0}\right) \rightarrow \operatorname{Aut}\left(\vee^{k} S^{1}\right) \rightarrow V_{k}
$$

определены с помощью функтора надстройки. Они индуцируют гомоморфизмы

$$
\operatorname{Aut}\left(\bigvee^{k} S^{0}\right) \rightarrow \pi_{0} \operatorname{Aut}\left(\bigvee^{k} S^{1}\right) \rightarrow \pi_{0} V_{k}
$$

$H$-пространство Aut $\left(\bigvee^{k} S^{1}\right)$ гомотопически эквивалентно Aut $F_{k}$, что определяет гомоморфизм $\chi:$ Aut $F_{k} \rightarrow \pi_{0} V_{k}$ как композицию

$$
\text { Aut } F_{k} \cong \pi_{0} \operatorname{Aut}\left(\vee^{k} S^{1}\right) \rightarrow \pi_{0} V_{k} \text {. }
$$

Отображение

$$
\pi_{0} \operatorname{Aut}\left(\bigvee^{k} S^{n}\right) \rightarrow \operatorname{Aut}\left(H_{n}\left(\bigvee^{k} S^{n} ; \mathbb{Z}\right)\right) \cong G L_{k} \mathbb{Z}
$$


индуцирует гомоморфизм $\pi_{0} V_{k} \rightarrow G L_{k} \mathbb{Z}$ такой, что композиция

$$
\text { Aut } F_{k} \stackrel{\chi}{\longrightarrow} \pi_{0} V_{k} \longrightarrow G L_{k} \mathbb{Z}
$$

равна $\rho_{k}$. Гомоморфизм $\chi$ порождает структуру алгебры над симметрической группой и две структуры алгебры над косами для системы групп $\pi_{0} V_{n}$.

Другой пример системы моноидов, которая возникает не из групп, дается системой моноидов Баеса-Бирман, рассматривавшейся в $\S 5$. Гомоморфизм $j_{n}(\S 5)$ из групшы кос $B r_{n}$ в $S B_{r}$ и спаривание $\mu$ (см. $\left.\S 12\right)$ определяют структуру алгебры над косами для системы моноидов $S B_{n}$.

Гомоморфизм $h_{n}: S B_{n} \rightarrow \overline{B P}_{n}$ определяется по формулам $h_{n}\left(g_{i}\right)=\sigma_{i}, h_{n}\left(a_{i}\right)=\xi_{i}$. Композиция $h_{n} \circ j_{n}$ равна каноническому гомоморфизму $\kappa_{n}: B r_{n} \rightarrow \overline{B P}_{n}$. Система представлений для редуцированной групш кос-перестановок определяет представления для моноидов $S B_{n}$, согласованные со структурой алгебры над косами.

Предположим теперь, что сушествует гомоморфизм $f$ из кольца $K$ в вешественные числа $\mathbb{R}$, отображаюший единицу $K$ в единицу $\mathbb{R}$. Тогда $f$ порождает гомоморфизм

$$
G L_{n} f: G L_{n} K \rightarrow G L_{n} \mathbb{R}
$$

Обозначим его для краткости $f_{n}$. Имеется каноническая ретракция $r_{n}$ из классифицирующего пространства полной линейной групшы над вещественными числами в классифицирующее пространство ортогональной группы

$$
r_{n}: B G L_{n} \mathbb{R} \rightarrow B O_{n}
$$

Полезна следующая классическая лемма о $r_{n}$.

ЛЕмма 13.1. Диаграмма

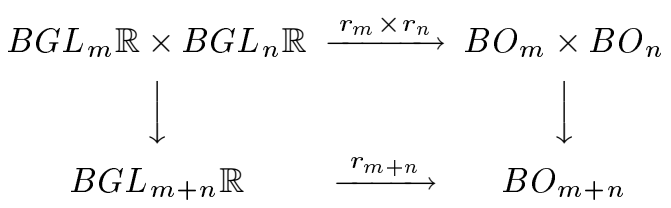

в которой вертикальные стрелки являются каноническими вложсениями, гомотопически коммутативна.

Пусть $\left\{H_{n}\right\}$ - модульная над косами система моноидов, согласованная с представлениями $\rho_{n}$. Рассмотрим функтор классифищируюшего пространства $B$ [67], [87], [88], [89] для моноида $H_{n}$ и возьмем композицию

$$
r_{n} B f_{n} B \rho_{n}: B H_{n} \rightarrow B G L_{n} K \rightarrow B G L_{n} \mathbb{R} \rightarrow B O_{n} .
$$

Она индуцирует векторное расслоение

$$
F_{n} \longrightarrow E_{n} \stackrel{p}{\longrightarrow} B H_{n} .
$$


Добавим теперь еще одно отображение $B \mu_{m, n}$ к композиции $(13.6)$ при $m+n$ :

$$
r_{m+n} B f_{m+n} B \rho_{m+n} B \mu: B B r_{m} \times B H_{n} \rightarrow B H_{m+n} \rightarrow B G L_{m+n} \mathbb{R} \rightarrow B O_{m+n} .
$$

Из леммы 13.1 получаем коммутативную диаграмму

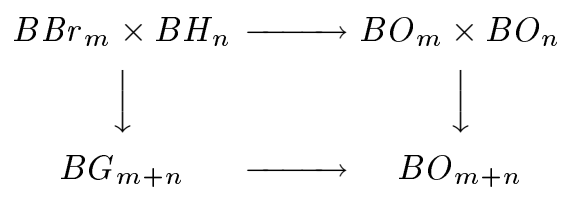

в которой левая нижняя композиция есть отображение (13.7). Каноническое представление групшы кос в ортогональную группу $O_{n}$ порождает отображение $\mathrm{BBr}_{n} \rightarrow B \mathrm{O}_{n}$. Применим функтор пространства Тома к этому отображению, получим:

$$
M \mathrm{Br}_{n} \rightarrow M \mathrm{O}_{n}
$$

Следующая теорема основывается на теореме М. Маховальда (теорема 13.1) и теореме Г. Сегала (теорема 8.11) и была доказана Ф.Р. Коэном [30] и Ш. Буллетом [43].

Теорема 13.3. Спектр Тома $\left\{\mathrm{MBr}_{n}\right\}$ изоморфен спектру Эйленберга-Маклейна для группь $\mathbb{Z} / 2$ :

$$
M B r_{n} \cong H \mathbb{Z} / 2 .
$$

Применим функтор пространства Тома к композициям (13.6) и (13.7). Получаем:

$$
\begin{gathered}
M r_{n} M f_{n} M \rho_{n}: M H_{n} \rightarrow M G L_{n} K \rightarrow M G L_{n} \mathbb{R} \rightarrow M O_{n}, \\
M r_{m+n} M f_{m+n} M \rho_{m+n} M \mu: M B r_{m} \wedge M H_{n} \rightarrow M H_{m+n} \rightarrow M G L_{m+n} \mathbb{R} \rightarrow M O_{m+n} .
\end{gathered}
$$

Наши условия на систему моноидов $H_{n}$ означают, что спектр Тома $M H$ становится модульным над спектром Тома групп кос, т.е. над $H \mathbb{Z} / 2$. Хорошо известно, что такой спектр сам есть букет надстроек над $H \mathbb{Z} / 2$. Если система моноидов $H_{n}$ является моноидальной и эта структура согласована со структурой модуля над косами и системой представлений, то спектр $M H$ становится мультипликативным спектром. Предположим дополнительно, что $M H$ коммутативен и $D$ есть мультипликативный дискретньй спектр (букет сфер) такой, что $H_{*}(D ; \mathbb{Z} / 2) \cong \pi_{*}(M H)$ как кольца. Тогда мы строим мультипликативный морфизм спектров $H \mathbb{Z} / 2 \wedge D \rightarrow M H$ как композицию

$$
H \mathbb{Z} / 2 \wedge D \longrightarrow M H \wedge M H \stackrel{\mu}{\longrightarrow} M H .
$$

Получаем следующую теорему.

Теорема 13.4. Спектр Тома МН системы моноидов, модульной над косами, с системой представлений, согласованной с модульной над косами структурой, эквивалентен букету надстроек над спектром Эйленберга-Маклейна для группь $\mathbb{Z} / 2$. Если система моноидов $H_{n}$ имеет моноидальную структуру и эта структура согласована с двумя предьдущими, то спектр МН мультипликативен. Если, дополнительно, МН коммутативен, то он эквивалентен смәш-произведению $H \mathbb{Z} / 2$ с некоторым мультипликативньлм дискретным спектром D:

$$
M H \simeq H \mathbb{Z} / 2 \wedge D
$$


СлеДСТвИЕ 13.1. Спектр Тома МН симметрически модульной системы моноидов с системой представлений, согласованной с симметрически модульной структурой, әквивалентен букету надстроек над спектром Эйленберга-Маклейна для группь $\mathbb{Z} / 2$. Спектр $M H$ есть модульный спектр над спектром Тома

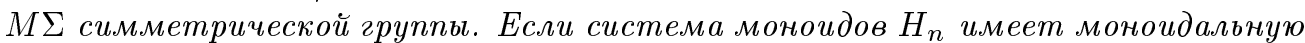
структуру и эта структура согласована с двумя предьдущими, то спектр МН мультипликативен. Если, дополнительно, $М$ н коммутативен, то он әквивалентен смәи-произведению $H \mathbb{Z} / 2$ с некоторым мультипликативным дискретным спектром D:

$$
M H \simeq H \mathbb{Z} / 2 \wedge D
$$

Теорема 13.4 и вычисления гомологий групп, проделанные в $\S 8,9,11$ и 12 позволяют вычислить спектры Тома для групп, обсуждавшихся ранее в настояшей работе. Это проделано в [61] и [86].

Э. Браун и $\Phi$. Петерсон [90], а также Р. Л. Коэн [91] изучали спектры Тома $M B r_{k}$ для фиксированных $k$. Пусть $B(l)$ обозначает спектр Брауна-Джитлера [92]. Э. Браун и $\Phi$. Петерсон [90] доказали следующий факт.

Теорема 13.5. Спектр Тома $\mathrm{MBr}_{k}$ 2-эквивалентен спектру Брауна-Джитлера $B([k / 2])$, где $[a]$ обозначает челую часть числа $а$.

СЛЕДСТВИЕ 13.2. Если морфизм

$$
t_{n}: M B r_{n} \rightarrow K(\mathbb{Z} / 2)
$$

представляет образующую когомологий $\mathrm{MBr}_{n}$ как модуля над алгеброй Стинрода, а $X$ - любой $C W$-комплекс, то соответствующий морфизм обобщенных теорий гомологий

$$
\left(M B r_{n}\right)_{q}(X) \rightarrow H_{q}(X, \mathbb{Z} / p)
$$

сюрвективен для $q \leqslant 2[n / 2]+1$.

Для нечетных простых Р. Л. Коэн [91] доказал следующий факт.

Теорема 13.6. Для $p>2 M_{k p}(k \not \equiv 0 \bmod p)$ гомотопически $p$-эквивалентен $(p-2) k$-й надстройке над спектром Брауна-Джитлера $S^{(p-2) k} B([k / p], p)$. Если морфизм

$$
s_{k}: M B r_{k p} \rightarrow K(\mathbb{Z} / p,(p-2) k)
$$

представляет образующую когомологий $\mathrm{MBr}_{k p}$ как модуля над алгеброй Стинрода, а $X$ - любой $C W$-комплекс, то соответствующий морфизм обобщенных теорий гомологий

$$
M B r\left(C_{k p}\right)_{q+(p-2) k}(X) \rightarrow H_{q}(X, \mathbb{Z} / p)
$$

сюрвективен для

$$
q \leqslant \begin{cases}2 p([k / p]+1)-1, & \text { если } k \neq 0 \bmod p \\ 2 k-1, & \text { если } k \equiv 0 \bmod p .\end{cases}
$$


Пусть $\Lambda_{p}$ есть $\Lambda$-алгебра по модулю $p$, описанная в [93]. Таким образом, $\Lambda_{2}$ есть градуированная $\mathbb{Z} / 2$-алгебра, порожденная элементами $\lambda_{i}$ степени $i$ для $i \geqslant 0$, подчиненными определенным соотношениям. Если $p$ нечетно, $\Lambda_{p}$ есть градуированная $\mathbb{Z} / p$-алгебра, порожденная элементами $\lambda_{i-1}$ степени $2 i(p-1)-1$ для $i \geqslant 1$ и элементами $\mu_{i-1}$ степени $2 i(p-1)$ для $i \geqslant 0$, также подчиненными определенным соотношениям. Пусть $J_{k}$ есть левьй идеал алгебры $\Lambda_{p}$, порожденный $\lambda_{0}, \ldots, \lambda_{k-1}$, если $p=2$, и $\lambda_{0}, \ldots, \lambda_{k-1}, \mu_{-1}, \ldots, \mu_{k-1}$, если $p$ нечетно. Тогда из результатов работ [91], [93] получаем следуюшие факты.

СЛЕДСТВИЕ 13.3. 2-локализачия гомотопической группь $\pi_{q}\left(M B r_{n}\right)$ изоморфна $\left(\Lambda_{2} / J_{[n / 2]}\right)_{q} \quad$ при $q<2[n / 2] ;$ р-локализачия гомотопической группы $\pi_{q}\left(M B r\left(C_{k p}\right)\right), k \not \equiv 0 \bmod p$, изоморфна $\left(\Lambda_{p} / J_{[k / p]}\right)_{q-(p-2) k}$ nри $q<p(2[k / p]+k+2)$ $-2(k+1)$.

\section{§14. Косы, монополи и пространства рациональных функций}

Пусть $\mathrm{Rat}_{k}$ есть пространство рациональных функций (степени $k$ )

$$
f: S^{2} \rightarrow S^{2}
$$

нормализованных предположением, что $f$ имеет $k$ полюсов и $f(\infty)=0$. Такая функция $f(z)$ может быть записана единственным образом в виде

$$
f(z)=\frac{a_{1} z^{k-1}+\cdots+a_{k}}{z^{k}+b_{1} z^{k}+\cdots+b_{k}}=\frac{h(z)}{m(z)},
$$

где полиномы $h(z)$ и $m(z)$ не имеют общих корней. $\Phi$. Р. Коэн, Р. Л. Коэн, Б. М. Манн и Р. Дж. Мильграм доказали следующий факт [44].

Tеорема 14.1. Пространство $\mathrm{Rat}_{k}$ стабильно гомотопически әквивалентно классифичирующему пространству группь кос из $2 k$ нитей $B r_{2 k}$ :

$$
\operatorname{Rat}_{k} \simeq_{S} B B r_{2 k} .
$$

Это означает, что существует гомотопическая әквивалентность между итерированныции надстройками этих пространств

$$
\Sigma^{N} \operatorname{Rat}_{k} \simeq \Sigma^{N} B \mathrm{Br}_{2 k}
$$

для достаточно больших $N$.

Пусть $\mathfrak{s u}(2)$ есть алгебра Ли группы Ли $S U(2)$. Обозначим через $A$ связность на тривиальном $S U(2)$-расслоении над $\mathbb{R}^{3}: \mathbb{R}^{3} \times S U(2)$, через $\varphi$ - $\mathfrak{s u}(2)$-значную функцию на $\mathbb{R}^{3}$. Пространство $\mathscr{A}$ пар $(A, \varphi)$, имеюших конечную энергию и удовлетворяюших условию

$$
1-|\varphi| \in L^{6}\left(\mathbb{R}^{3}\right),
$$

рассматривалось в [94] и [46] в связи с уравнениями Янга-Миллса-Хиггса. К.Г. Таубс доказал следуюший факт [94]. 
ТЕОРема 14.2. Существует гомотопическая әквивалентность

$$
I: \Omega^{2} S^{2} \rightarrow \mathscr{A}
$$

Пусть $\mathscr{G}$ есть группа калибровочньх преобразований:

$$
\mathscr{G}=\operatorname{Map}_{0}\left(\mathbb{R}^{3}, S U(2)\right)
$$

где $\mathrm{Map}_{0}$ означает отображения

$$
g: \mathbb{R}^{3} \rightarrow S U(2)
$$

удовлетворяющие условию

$$
\lim _{t \rightarrow \infty} g(t, 0,0)=(1,0,0)
$$

Группа $\mathscr{G}$ стягиваема. Она действует на $\mathscr{A}$, определяя фактор-пространство

$$
\mathscr{B}=\mathscr{A} / \mathscr{G}
$$

Пространство $\mathscr{A}$ есть пространство главного расслоения со слоем $\mathscr{G}$. Поскольку $\mathscr{G}$ стягиваема, заключаем, что проекция

$$
\mathscr{A} \rightarrow \mathscr{B}
$$

есть гомотопическая эквивалентность.

СлЕДСтвиЕ 14.1. Существует естественная гомотопическая әквивалентHocms

$$
\mathscr{B} \rightarrow \Omega^{2} S^{2}
$$

Теорема 14.2 и следствие 14.1 означают, что пространства $\mathscr{A}$ и $\mathscr{B}$ состоят из компонент связности, заиндексованных целыми числами $\mathbb{Z}$ :

$$
\mathscr{A}_{k} \simeq \mathscr{B}_{k} \rightarrow \Omega_{k}^{2} S^{2}
$$

Это число $k$ назьвается зарядом пары $(A, \varphi)$.

Пусть $\mathscr{M}_{k}$ есть пространство модулей отмеченных $S U(2)$-монополей в $\mathbb{R}^{3}$ заряда $k$ (определения см., например, в [95]). Оно является подпространством в $\mathscr{B}_{k}$ :

$$
\mathscr{M}_{k} \subset \mathscr{B}_{k}
$$

Пространство $\mathscr{M}_{k}$ есть гладкое многообразие размерности $4 k$ и $\mathscr{M}_{1}=S^{1} \times \mathbb{R}^{3}$.

К. Г. Таубс доказал также следуюшую теорему [94]. 
Tеорема 14.3. Вложение $i: \mathscr{M}_{k} \rightarrow \mathscr{B}_{k}$ является асимптотической гомотопической эквивалентностью, что означает, что существует функиия $q(k), \partial л я$ которой

$$
\lim _{k \rightarrow \infty} q(k)=\infty
$$

и отображсение $і$ индуцирует изоморфизм гомотопических групп $\pi_{q} n p u q \leqslant q(k)$.

Таким образом, пространство $\mathscr{M}_{k}$ есть конечномерная гомотопическая аппроксимация пространства $\Omega_{k}^{2} S^{2}$, а в $\S 8$ мы замечали, что конфигурационное пространство $B\left(\mathbb{R}^{2}, k\right)$ есть конечномерная гомологическая аппроксимация того же пространства.

C. К. Дональдсон установил следующую связь между пространствами $\mathscr{M}_{k}$ и $\mathrm{Rat}_{k}$ (см. [45]).

Tеорема 14.4. Многообразие $\mathscr{M}_{k}$ диффеоморфно $\operatorname{Rat}_{k}$ :

$$
\mathscr{M}_{k} \cong \mathrm{Rat}_{k}
$$

Теоремы 14.1 и 14.4 означают, что три пространства: пространство монополей $\mathscr{M}_{k}$, пространство рациональных функций Rat ${ }_{k}$ и классифицируюшее пространство группы кос из $2 k$ нитей $B B r_{2 k}$, имеют те же самые обобщенные гомологии и когомологии:

$$
\begin{aligned}
h^{*}\left(\mathscr{M}_{k}\right) & \cong h^{*}\left(B B r_{2 k}\right), \\
h^{*}\left(\operatorname{Rat}_{\mathrm{k}}\right) & \cong h^{*}\left(B B r_{2 k}\right)
\end{aligned}
$$

для любой теории $h^{*}(\cdot)$.

Теперь пусть $\operatorname{Rat}_{k}^{0}$ есть подпространство пространства $\mathrm{Rat}_{k}$, состоящее из рациональных функций с $k$ различными простыми полюсами. Если $f \in \operatorname{Rat}_{k}^{0}$, то она может быть записана в виде

$$
f=\sum_{i=1}^{k} \frac{a_{i}}{z_{i}-b_{i}}
$$

где $b_{i}$ - различные комплексные числа, а $a_{i}$-ненулевые комплексные числа. Следовательно,

$$
\operatorname{Rat}_{k}^{0}=F(\mathbb{C}, k) \times_{\Sigma_{k}}\left(\mathbb{C}^{*}\right)^{k},
$$

где $\mathbb{C}^{*}$ есть пространство ненулевых комплексных чисел. Симметрическая группа действует на обоих пространствах $F(\mathbb{C}, k)$ и $\left(\mathbb{C}^{*}\right)^{k}$ перестановками. Отсюда следует, что $\mathrm{Rat}_{k}^{0}$ есть классифицциующее пространство группы $R b_{k}$ ленточны $x$, или осн $a^{-}$ щенных, кос, являющейся сплетением $R b_{k}=B r_{k} \int \mathbb{Z}$. Группа $R b_{k}$, естественным образом, является подгруппой группы $B r_{2 k}$. Вложение

$$
R b_{k} \rightarrow B r_{2 k}
$$

задается процессом "кабелирования", описьваемым следующим образом. Возьмем $k$ пар нитей и перекрутим $i$-ю пару $n_{i}$ раз. Затем сплетем эти $k$ пар в соответствии $c$ косой $b \in B r_{k}$. Это дает косу из $2 k$ нитей, и отображение, переводяшее $\left(b ; n_{1}, \ldots, n_{k}\right)$ в полученную косу, есть необходимое вложение (14.1). 
Таким образом, имеются два следующих отображения из пространства $B R b_{k}=$ $\operatorname{Rat}_{k}^{0}$ :

$$
\begin{gathered}
\phi: B R b_{k} \rightarrow B b r_{2 k}, \\
\psi: \operatorname{Rat}_{k}^{0} \rightarrow \operatorname{Rat}_{k}=\mathscr{M}_{k} .
\end{gathered}
$$

Р. Л. Коэн и Дж. Д. С. Джонс описали индуцированное отображение в обобщенных когомологиях [46].

ТЕОРема 14.5. Для любой обобщенной теории когомологий $h^{*}(\cdot)$ отображения

$$
\begin{gathered}
\phi^{*}: h^{*}\left(B B r_{2 k}\right) \rightarrow h^{*}\left(B R b_{k}\right), \\
\psi^{*}: h^{*}\left(\mathscr{M}_{k}\right) \rightarrow h^{*}\left(B R b_{k}\right)
\end{gathered}
$$

оба иньективны и имеют один и тот же образ. Следовательно, имеется естественный изоморфизм

$$
\left(\phi^{*}\right)^{-1} \psi^{*}: h^{*}\left(\mathscr{M}_{k}\right) \rightarrow h^{*}\left(B B r_{2 k}\right) .
$$

Для пары $c=(A, \varphi) \in \mathscr{A}$ имеется вещественньй оператор Дирака $\delta_{c}$. К. Г. Таубс доказал, что этот оператор расширяется до Фредгольмова оператора в подходящих пространствах Соболева [94]. Более того, если две пары $c_{1}$ и $c_{2}$ калибровочно эквивалентны, то операторы $\delta_{c_{1}}$ и $\delta_{c_{2}}$ изоморфны. Таким образом, мы получаем семейство операторов, параметризованное точками $c \in \mathscr{B}$. К. Г. Таубс доказал, что это семейство непрерьвно, и, следовательно, определено индексное расслоение

Отображение (13.1)

$$
\operatorname{ind}(\delta) \in K O^{0}(\mathscr{B}) \text {. }
$$

$$
\Omega^{2} S^{2} \rightarrow \mathbb{Z} \times B O
$$

задает элемент $\gamma \in K O^{0}\left(\Omega^{2} S^{2}\right)$ с соответствуюшими компонентами $\gamma_{k} \in K O^{0}\left(\Omega_{k}^{2} S^{2}\right)$.

Tеорема 14.6 [46]. При естественном изоморфизме $K O^{0}\left(\mathscr{B}_{k}\right)$ и $K O^{0}\left(\Omega_{k}^{2} S^{2}\right)$ индексное расслоение ind $(\delta)$ отожсдествляется $c \gamma_{k}$ :

$$
\begin{gathered}
K O^{0}\left(\mathscr{B}_{k}\right) \cong K O^{0}\left(\Omega_{k}^{2} S^{2}\right), \\
\operatorname{ind}(\delta) \leftrightarrow \gamma_{k} .
\end{gathered}
$$

Пусть $\pi_{k}: R b_{k} \rightarrow O_{k}$ есть композиция

$$
R b_{k}=B r_{k} \int \mathbb{Z} \rightarrow \Sigma_{k} \int \mathbb{Z} / 2 \hookrightarrow O_{k},
$$

где первое отображение индуцировано двумя эпиморфизмами: $B r_{k} \rightarrow \Sigma_{k}$ и $\mathbb{Z} \rightarrow \mathbb{Z} / 2$, а второе является каноническим вложением $\Sigma_{k} \int \mathbb{Z} / 2$ как группы Кокстера $C_{k}$ в $O_{k}$. Это представление определяет расслоение, которое мы будем обозначать также через $\pi_{k}$. Это расслоение может быть описано следуюшим образом. Пусть $H$ есть пространство вешественного расслоения Хопфа над $\mathbb{C}^{*}=\mathbb{R} \times S^{1}$, тогда $\pi_{k}$ есть расслоение

$$
F(\mathbb{C}, k) \times_{\Sigma_{k}}(H)^{k} \rightarrow F(\mathbb{C}, k) \times_{\Sigma_{k}}\left(\mathbb{C}^{*}\right)^{k} .
$$

Расслоение $\pi_{k}$ имеет такое наглядное описание: его слой над конфигурацией из $k$ точек - это пространство всех $\mathbb{R}$-значных функций на этом множестве.

Предположим, что $c \in \mathscr{M}_{k}$, тогда индексное расслоение ind $(\delta)$ определено над пространством монополей $\mathscr{M}_{k}$. 
TеОрема 14.7. Образ индексного расслоения ind $(\delta)$ при отображсении $\psi^{*}$ совпадает с расслоением, индуцированным отображением $\pi_{k}$ :

$$
\psi^{*}(\operatorname{ind}(\delta))=\pi_{k} \in K O^{0}\left(B R b_{k}\right) .
$$

Доказательство этой теоремы было дано в [47]. Для $k=1 \mathscr{M}_{1}=\mathbb{R}^{3} \times S^{1}$ и $\operatorname{ind}(\delta)$ есть нетривиальное линейное расслоение над $\mathscr{M}_{1}$, т.е. вещественное линейное расслоение Хопфа над окружностью $S^{1}$.

Пусть $q: R b_{k} \rightarrow B r_{k}$ есть канонический гомоморфизм факторизации. Отображение

$$
i^{0}: K O^{0}\left(B B r_{2 k}\right) \rightarrow K O^{0}\left(B B r_{k}\right),
$$

индуцированное вложением $i: B r_{k} \hookrightarrow B r_{2 k}$, расщепляется. Более точно, доказан следующий факт [46].

ПРЕДЛОЖЕНИЕ 14.1. Имеется естественное отображение

$$
\alpha: K O^{0}\left(B B r_{k}\right) \rightarrow K O^{0}\left(B B r_{2 k}\right)
$$

maкое, что

i) $i^{0} \alpha=1$,

ii) $j^{0} \alpha=q^{0}$.

Пусть $\rho_{k}: B r_{k} \rightarrow O_{k}$ есть представление через перестановки. Отображение $\alpha$ позволяет охарактеризовать индексное расслоение над $\mathscr{M}_{k}[46]$.

ТеОРема 14.8. Используя естественньй изоморфизм $K O^{0}\left(\mathscr{M}_{k}\right)$ и $K O^{0}\left(B B r_{2 k}\right)$, индексное расслоение ind $(\delta)$ мохсет бъть отожсдетвлено с $\rho_{2 k}-\alpha\left(\rho_{2 k}\right)$ :

$$
\begin{gathered}
K O^{0}\left(\mathscr{M}_{k}\right) \cong K O^{0}\left(B B r_{2 k}\right), \\
\operatorname{ind}(\delta) \leftrightarrow \rho_{2 k}-\alpha\left(\rho_{2 k}\right)
\end{gathered}
$$

\section{Дополнение. Категории и пространства петель}

Здесь мы собрали некоторые понятия и факты, которые могут быть полезны при чтении основного текста обзора. Очень хорошими ссылками являются работы Ф. Адамса [66] и П. Мэя [96].

Вспомним [56], что моноидальная (или тензорная) категория $\mathscr{V}=(\mathscr{V}, \otimes, I, a, l, r)$ состоит из категории $\mathscr{V}$, функтора $\otimes: \mathscr{V} \times \mathscr{V} \rightarrow \mathscr{V}$, называемого произведением (или тензорным произведением), объекта $I \in \mathcal{V}$, называемого единичным облектом, и естественных эквивалентностей

$$
\begin{gathered}
a=a_{A, B, C}:(A \otimes B) \otimes C \stackrel{\sim}{\longrightarrow} A \otimes(B \otimes C), \\
l=l_{A}: I \otimes A, \quad r=r_{A}: A \otimes I A,
\end{gathered}
$$

назьваемых ассоциативностью, левой единицей и правой единицей соответственно, таких что для любых объектов $A, B, C, D \in \mathscr{V}$ следующие две диаграммы, называемые пятиугольником ассоциативности и треугольником для единицы, коммутируют: 


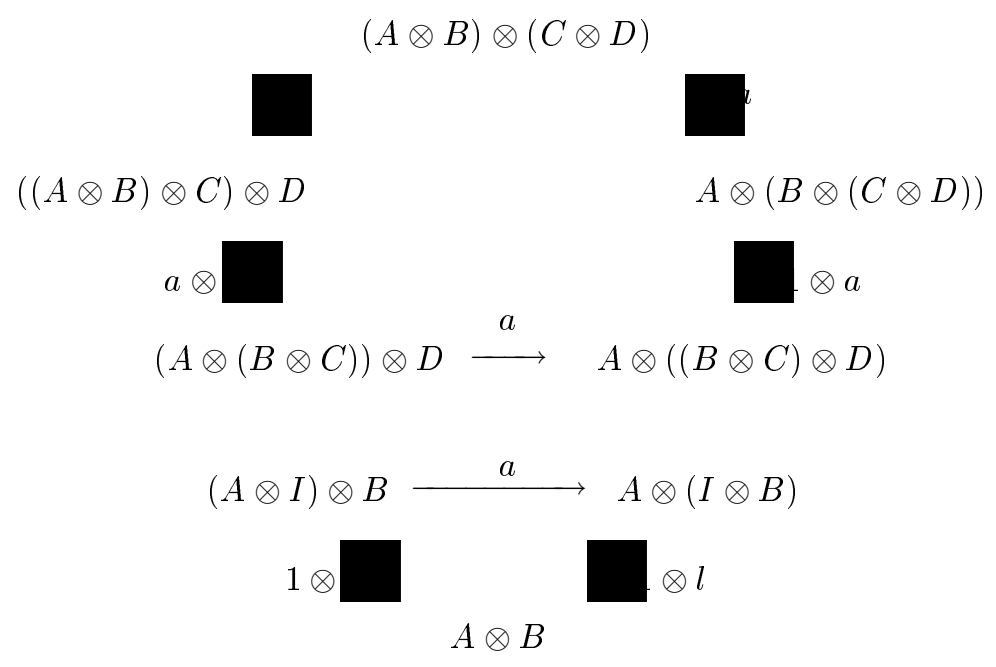

Тензорная категория называется строгой, когда все морфизмы $a_{A, B}, l_{A}, r_{A}$ являются тождественными.

Заплетение (англ. - braiding) в моноидальной категории $\mathscr{V}$ состоит из семейства естественных эквивалентностей

$$
c=c_{A, B}:(A \otimes B) \stackrel{\sim}{\longrightarrow} B \otimes A
$$

в $\mathcal{V}$ таких, что две диаграммы (B1) и (B2) коммутируют:

(B1)

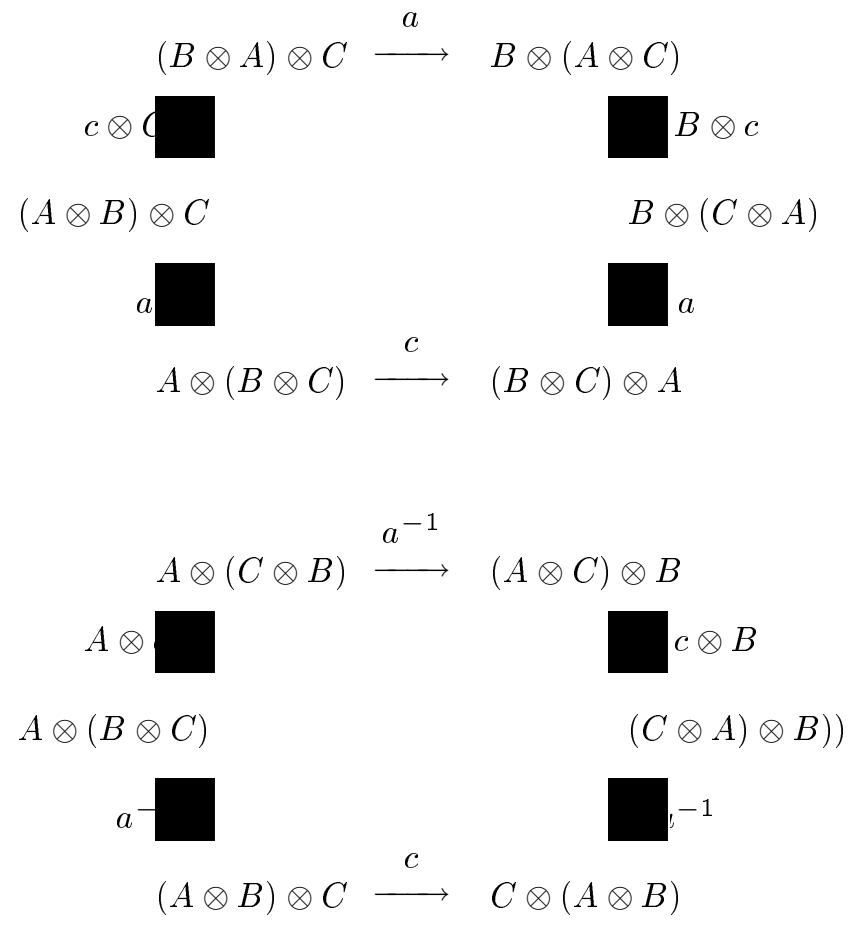


Если $c$ - заплетение, то таковым же будет и $c^{\prime}$, заданное по формуле $c^{\prime}=c_{A, B}^{-1}$. Симметрией называется заплетение, для которого $c=c^{\prime}$.

Моноидальная категория с заплетением (англ. - braided monoidal category) или просто категория $c$ заплетением, есть пара $(\mathscr{V}, c)$, состояшая из моноидальной категории и заплетения.

Симметрическая моноидальная категория есть пара $(\mathscr{V}, s)$, состоящая из моноидальной категории и симметрии. Пермутативная категория есть строгая симметрическая моноидальная категория.

Примеры моноидальных категорий с заплетением и симметрических моноидальных категорий имеются в основном тексте обзора. Главным примером моноидальной категории с заплетением является категория, образованная группами кос $\mathscr{B}$. Следуюшая система элементов:

$$
\sigma_{m} \ldots \sigma_{1} \sigma_{m+1} \ldots \sigma_{2} \ldots \sigma_{n+m-1} \ldots \sigma_{n} \in B r_{m+n}
$$

определяет заплетение $c$ в $\mathscr{B}$. Графически такой элемент изображен на рис. Д.1.

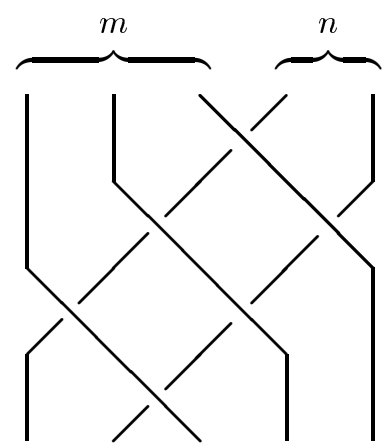

Рис. Д.1

Пусть $\mathscr{P}(T)$ обозначает множество подмножеств множества $T$, а $\mathbf{n}$ обозначает множество $\{1,2, \ldots, n\}$. Г. Сегал [71] определяет $Г$ как категорию всех конечных множеств, морфизмами которой из $S$ в $T$

$$
S \rightarrow T
$$

являются отображения

$$
\theta: S \rightarrow \mathscr{P}(T)
$$

такие, что $\theta(\alpha)$ и $\theta(\beta)$ не пересекаются, если $\alpha \neq \beta$. Композиция $\theta: S \rightarrow \mathscr{P}(T)$ и $\phi: T \rightarrow \mathscr{P}(U)$ есть

$$
\psi: S \rightarrow \mathscr{P}(U)
$$

где $\psi(\alpha)=\bigcup_{\beta \in \theta(\alpha)} \psi(\beta)$.

Г-категорией называется контравариантньй функтор $\mathscr{E}$ из Г в категории такой, чTO

(i) $\mathscr{E}(\mathbf{0})$ эквивалентно категории с одним объектом и одним морфизмом; 
(ii) для каждого $n$ функтор

$$
p_{n}: \mathscr{E}(\mathbf{n}) \rightarrow \underbrace{\mathscr{E}(\mathbf{1}) \times \cdots \times \mathscr{E}(\mathbf{1})}_{n \text { раз }}
$$

индуцированньй морфизмом $i_{k}: \mathbf{1} \rightarrow \mathbf{n}$ в $Г$, где $i_{k}(1)=k \subset \mathbf{n}$, является эквивалентностью категорий.

Мы определяем $c n е к т р ~ E$ как последовательность пространств $E_{i}$, гомеоморфных пространству петель $\Omega E_{i+1}$. Отображениями спектров являются последовательности $f_{i}: D_{i} \rightarrow E_{i}$ такие, что $f_{i}$ согласуется с $\Omega f_{i+1}$ при помоши указанного семейства гомеоморфизмов. Пространства, имеющие гомотопический тип некоторого пространства $E_{0}$ из гомотопического класса некоторого $f_{0}$, называются бесконечно кратными пространствами петель и бесконечно кратными петлевыми отображсениями.

Имеется функтор стабилизации $Q$ из пространств в пространства, определенньй по формуле

$$
Q X=\lim _{\rightarrow} \Omega^{j} S^{j} X, \quad \pi_{n} Q X=\pi_{n}^{S} X
$$

где $S$ есть функтор надстройки, а $\pi_{*}^{S} X$ - стабильные гомотопические группы пространства $X$. Имеется также функтор стабилизации $Q_{\infty}$ из пространств в спектры, определенньй по формуле

$$
\left(Q_{\infty} X\right)_{i}=Q S^{i} X=\underline{\lim } \Omega^{j} S^{i+j} X
$$

Поскольку $E_{0} \cong \Omega^{n} E_{n}$, это дает $n$ различных координат, которые могут быть использованы для определения произведения, и все эти произведения между собой гомотопны. Таким образом, возникает пространство произведений, пространство трехкратных произведений $E_{0} \times E_{0} \times E_{0} \rightarrow E_{0}$, и т. д. В этих пространствах закодирована внутренняя структура $E_{0}$, происходящая из $E_{i}$. Подходящий формализм выражен в понятии операды. Операда $\mathscr{C}$ состоит из пространств $\mathscr{C}(j), j \geqslant 0$, которые надо мыслить как пространства $j$-арных операций, $\mathscr{C}(0)$ есть точка, $\mathscr{C}(1)$ содержит единичный элемент 1. Имеется правое действие симметрической группы $\Sigma_{j}$ на пространстве $\mathscr{C}(j), j \geqslant 0$, и имеются отображения

$$
\gamma: \mathscr{C}(k) \times \mathscr{C}\left(j_{1}\right) \times \cdots \times \mathscr{C}\left(j_{k}\right) \rightarrow \mathscr{C}\left(j_{1}+\cdots+j_{k}\right)
$$

Введенные объекты подчинены некоторому числу аксиом, которые можно найти в [88].

Действие $\theta$ операды $\mathscr{C}$ на пространстве $X$ состоит из $\Sigma_{j}$-эквивариантных отображений

$$
\mathscr{C}(j) \times X^{j} \rightarrow X
$$

где слева $\Sigma_{j}$ действует по диагонали, а справа тривиальньм образом. Эти отображения также подчинены некоторым естественным аксиомам [88]. Отображение $\theta_{0}$ является вложением базисной точки в $X$. Единица $1 \in \mathscr{C}(1)$ определяет тождественное отображение пространства $X$. Эквивариантность отображения $\theta_{j}$ согласована с перестановками переменных, а отображения $\gamma$ связаны с композицией. Пара $(X, \theta)$, состоящая из пространства и действия операды $\mathscr{C}$ на нем, называется $\mathscr{C}$-пространством. 
Пунктированное отображение $f: X \rightarrow X^{\prime}$ между двумя $\mathscr{C}$-пространствами $(X, \theta)$ и $\left(X^{\prime}, \theta^{\prime}\right)$ называется $\mathscr{C}$-отображением, если оно коммутирует с действием операд.

Операда $\mathscr{C}$ называется $E_{\infty}$-операдой, если каждое пространство $\mathscr{C}(j)$ стягиваемо согласовано со свободным $\Sigma_{j}$-действием. $E_{\infty}$-пространством называется $\mathscr{C}$-пространство, где $\mathscr{C}$ есть любая $E_{\infty}$-операда, и $\mathscr{C}$-отображение назьвается $E_{\infty}$-отображением в этом случае.

Г-пространство есть контравариантный функтор $A$ из Г в топологические пространства такой, что:

(i) $A(\mathbf{0})$ стягиваемо;

(ii) для любого $n$ отображение

$$
p_{n}: A(\mathbf{n}) \rightarrow \underbrace{A(\mathbf{1}) \times \cdots \times A(\mathbf{1})}_{n \text { раз }},
$$

индуцированное отображениями $i_{k}: \mathbf{1} \rightarrow \mathbf{n}$ в $Г$, где $i_{k}(1)=k \subset \mathbf{n}$, является гомотопической эквивалентностью.

$У_{\mathcal{M н о ж}}$ жние на $Г$-пространстве $A$ есть контравариантньй функтор $\hat{A}$ из $\Gamma \times \Gamma$ в топологические пространства вместе с естественньми преобразованиями

$$
\begin{gathered}
i_{1}: \hat{A}(S, T) \rightarrow A(S), \quad i_{2}: \hat{A}(S, T) \rightarrow A(T), \\
m: \hat{A}(S, T) \rightarrow A(S \times T),
\end{gathered}
$$

функториальньми по $S$ и $T$ в $\Gamma$, и такой, что отображение

$$
i_{1} \times i_{2}: \hat{A}(S, T) \rightarrow A(S) \times A(T)
$$

есть гомотопическая эквивалентность.

По аналогии со случаями моноидов или групп может быть определено классифицируюшее пространство категории $\mathscr{C}$ (см., например, [66]). Мы обозначаем его через $|\mathscr{C}|$. Для Г-категории $\mathscr{E}$ функтор

$$
S \rightarrow|\mathscr{E}(S)|
$$

обозначается через $|\mathscr{E}|$. Г. Сегал [71] доказал, что если $\mathscr{E}$ есть Г-категория, то $|\mathscr{E}|$ есть Г-пространство.

Операда кубиков $\mathscr{C}_{n}$ определяется для каждого $n$ следующим образом. Пусть $\mathscr{C}_{n}(j)$ есть пространство линейных вложений ("кубиков") $I^{n} \rightarrow I^{n}$ с параллельными осями. Пространства $\mathscr{C}_{n}(j)$ составляют операду кубиков $\mathscr{C}_{n}$, являюшуюся подоперадой $\mathscr{C}_{n+1}$. Прямой предел есть операда $\mathscr{C}_{\infty}$. Пространство $\mathscr{C}_{n}(j)$ является $\Sigma_{j}$-эквивариантно эквивалентным конфигурационному пространству наборов из $j$ различных точек в $\mathbb{R}^{n}$ и является $(n-2)$-связным, следовательно, $\mathscr{C}_{\infty}$ есть $E_{\infty}$-операда. Отображение дополнения кубиков в базисную точку определяет естественное действие операды кубиков $\mathscr{C}_{n}$ на $n$-кратных пространствах петель. Принцип распознавания утверждает, что обратное также верно. Чтобы сформулировать это более точно, нам понадобится понятие группового пополнения. Для $H$-пространства $X$ групповое 
пополнение есть $H$-отображение $g: X \rightarrow Z$ такое, что $\pi_{0} Z$ есть группа и индуцированньй гомоморфизм в гомологиях

$$
g_{*}: H_{*}(X ; k) \rightarrow H_{*}(Z ; k)
$$

является $\mathbb{Z}\left[\pi_{0} X\right]$-локализацией понтрягинского кольца $H_{*}(X ; k)$ для любого коммутативного кольца коэффищиентов $k$. Если $\mathbb{Z}\left[\pi_{0} X\right]$ само является группой, то $g$ есть эквивалентность.

Принцип распознавания утверждает, что если $X$ есть $E_{\infty}$-пространство, то имеется спектр $\mathbf{B} X=\left\{B_{i} X\right\}$ и $E_{\infty}$-отображение $\iota: X \rightarrow B_{0} X$, являюшееся групповым пополнением. Конструкция П. Мэя [88] основана на операдной технике. Аналогично, Г. Сегал [71] для каждого Г-пространства $A$ строит спектр, которьй мультипликативен, если Г-пространство $A$ было с умножением.

Первая часть следующей теоремы была доказана П. Мэем [67], а вторая - Р. В. Томасоном [97].

ТЕоРема Д.1. Классифичирующее пространство симметрической моноидальной категории становится бесконечнократным пространством петель после применения плюс-конструкиии Квиллена и все бесконечнократные пространства петель получаются таким способом.

Модифицируя доказательство П. Мэя [67] и доказательство Р. В. Томасона [97] теоремы Д.1 на случай категории с заплетением, можно доказать аналогичньй факт в этом случае. Это сделано З. Федоровичем [98].

ТЕОРемА Д.2. Классифицирующее пространство моноидальной категории с заплетением становится двукратным пространством петель после применения плюс-конструкиии Квиллена, и все двукратные пространства петель получаются таким способом. Функторы, сохраняющие заплетение, индуцируют двукратно петлевъе отображения плюс-конструкций классифицирующих пространств соответствующих категорий.

\section{СПИСОК ЛИТЕРАТУРЫ}

[1] Artin E. Theorie der Zöpfe // Abh. Math. Sem. Univ. Hamburg. 1925. V. 4. P. 47-72.

[2] Hurwitz A. Über Riemannische Flächen mit gegebenen Verzweigungspunkten // Math. Ann. 1891. V. 39. P. 1-61.

[3] Fricke R., Klein F. Vorlesungen über die Theorie der automorphen Functionen. Bd. I. Gruppentheoretischen Grundlagen. Leipzig: Teubner, 1897. (New York: Johnson Repr. Corp., 1965.)

[4] Artin E. Theory of braids // Ann. Math. 1947. V. 48. № 1. P. 101-126.

[5] Markoff A. A. Über die freie Äquivalenz der geschlossenen Zöpfe // Math. Sb. 1936. V. 16. P. 73-78.

[6] Birman J.S. Braids, Links, and Mapping Class Groups. Princeton: Princeton Univ. Press, 1975. (Ann. of Math. Stud. V. 82.)

[7] Jones V.F. R. Hecke algebra representations of braid groups and link polynomials // Ann. of Math. 1987. V. 126. P. 335-388.

[8] Turaev V.G. The Yang-Baxter equation and invariants of links // Invent. Math. 1988. V. 92. P. $527-553$.

[9] Прасолов В. В., Сосинский А. Б. Узлы, зацепления, косы и трехмерные многообразия. М.: МЦНМО, 1997. 
[10] Fox R., Neuwirth L. The braid groups // Math. Scand. 1962. V. 10. № 1. P. 119-126.

[11] Fadell E., Neuwirth L. Configuration spaces // Math. Scand. 1962. V. 10. № 1. P. 111-118.

[12] Арнольд В.И.О некоторых топологических инвариантах алгебраических функций // Труды ММО. 1970. Т. 21. С. 27-46.

[13] Васильев В. А. Топология дополнений к дискриминантам. М.: Фазис, 1997.

[14] Брискорн Э. О группах кос (по В.И. Арнольду) // Математика (сб. переводов). 1974. T. 18. № 3. C. $46-59$.

[15] Sossinsky A. B. Preparation theorems for isotopy invariants of links in 3-manifolds // Lecture Notes in Math. 1992. V. 1510. P. 354-362.

[16] Schechtman V. V., Varchenko A. N. Arrangements of hyperplanes and Lie algebra homology // Invent. Math. 1991. V. 106. P. 139-194.

[17] Lambropoulou S. Solid torus links and Hecke algebras of type B // Proc. Conference on Quantum Topology / ed. D. N. Yetter. Singapore: World Scientific Press, 1993. P. 225-245.

[18] Вершинин В. В. Инварианты Васильева и косы с особенностями // УМН. 1998. Т. 53. № 2. C. 141-142.

[19] Vershinin V. V. On Vassiliev invariants for links in handlebodies // J. Knot Theory Ramifications. 1998. V. 7. № 5. P. 701-712.

[20] Марков А. А. Основы алгебраической теории кос // Труды МИАН. 1945. Т. 16.

[21] Baez J. C. Link invariants of finite type and perturbation theory // Lett. Math. Phys. 1992. V. 26. P. $43-51$

[22] Birman J.S. New points of view in knot theory // Bull. Amer. Math. Soc. 1993. V. 28. № 2. P. 253-387.

[23] Fenn R., Rimányi R., Rourke C. Some remarks on the braid-permutation group // Topics in Knot Theory. Dordrecht: Kluwer Acad. Publ., 1993. P. 57-68.

[24] Fenn R., Rimányi R., Rourke C. The braid-permutation group // Topology. 1997. V. 36. № 1. P. 123-135.

[25] Арнольд В.И. Топологические инварианты алгебраических функций. II // Функцион. анализ и его прил. 1970. Т. 4. № 2. С. 1-9.

[26] А рнольд В. И.Кольцо когомологий группы крашеных кос // Матем. заметки. 1969. Т. 5. № 2. C. 227-231.

[27] Фукс Д. Б. Когомологии групп кос $\bmod 2 / /$ Функцион. анализ и его прил. 1970. Т. 4. № 2. C. $62-75$.

[28] Cohen F. Cohomology of braid spaces // Bull. Amer. Math. Soc. 1973. V. 79. № 4. P. $763-766$

[29] Cohen F. Homology of $\Omega^{n+1} \Sigma^{n+1} X$ and $C_{n+1} X, n>0$ // Bull. Amer. Math. Soc. 1973. V. 79. №6. P. 1236-1241.

[30] Cohen F. Braid orientations and bundles with flat connections // Invent. Math. 1978. V. 46. P. $99-110$.

[31] Cohen F. Artin's braid groups, classical homotopy theory, and other curiosities // Contemp. Math. 1988. V. 78. P. 167-206.

[32] Cohen F., Lada T., May J.P. The Homology of Iterated Loop Spaces. Berlin: Springer-Verlag, 1976. (Lecture Notes in Math. V. 533.)

[33] Вайнштейн Ф. В.Когомологии групп кос // Функцион. анализ и его прил. 1978. Т. 12. № 2. C. $72-73$.

[34] Горюнов В.В. Когомологии групп кос серий $C$ и $D$ и некоторые стратификации // Функцион. анализ и его прил. 1978. Т. 12. № 2. С. 76-77.

[35] Горюнов В.В. Когомологии групп кос серий $C$ и $D / /$ Труды ММО. 1981. Т. 42. C. $234-242$.

[36] Segal G. B. Configuration spaces and iterated loop spaces // Invent. Math. 1973. V. 21. P. 213-221.

[37] Barrat M. G. A Free group functor for stable homotopy // Proc. Sympos. Pure Math. 1971. № 22. P. 31-35. 
[38] Priddy S. B. On $\Omega^{\infty} S^{\infty}$ and the infinite symmetric group // Proc. Sympos. Pure Math. 1971. № 22. P. 217-220.

[39] Mahowald M. A new family in $\pi_{*}^{s} / /$ Topology. 1977. V. 16. P. 249-254.

[40] Mahowald M. Ring spectra which are Thom complexes // Duke Math. J. 1977. V. 46. № 3. P. 549-259.

[41] Salvetti M. Topology of the complement of real hyperplanes in $\mathbb{C}^{N} / /$ Invent. Math. 1987. V. 88. P. $603-618$.

[42] Salvetti M. The homotopy type of Artin groups // Math. Res. Lett. 1994. V. 1. P. 565-577.

[43] Bullett S. Permutations and braids in cobordism theory // Proc. London Math. Soc. 1979. V. 38. № 3. P. 517-531.

[44] Cohen F., Cohen R. L., Mann B. M., Milgram R. J. The topology of rational functions and divisors of surfaces // Acta Math. 1991. V. 166. P. 163-221.

[45] Дональдсон С. К. Уравнения Нама и классификация монополей // Монополи. Топологические и вариационные методы. Сб. статей. М.: Мир, 1989. С. 123-159.

[46] Cohen R. L., Jones J. D.S. Representations of braid groups and operators coupled to monopoles // London Math. Soc. Lecture Notes Ser. 1990. V. 150. P. 191-207.

[47] Cohen R. L., Jones J. D. S. Monopoles, braid groups, and the Dirac operators // Comm. Math. Phys. 1993. V. 158. № 2. P. 241-266.

[48] Garside F. A. The braid group and other groups // Quart. J. Math. Oxford Ser. (2). 1969. V. 20. P. $235-254$

[49] Брискорн Э., Сайто К. Группы Артина и группы Кокстера // Математика (сб. переводов). 1974. Т. 18. №6. С. 56-79.

[50] Dehornoy P. Groups with a complemented presentation // J. Pure Appl. Algebra. 1997. V. 116. № 1-3. P. 115-137.

[51] Dehornoy P. A fast method for comparing braids // Adv. Math. 1997. V. 125. № 2. P. $200-235$.

[52] Smale S. On the topology of algorithms. I // J. Complexity. 1988. V. 4. № 4. P. 81-89.

[53] Васильев В.А. Когомологии групп кос и сложность алгоритмов // Функцион. анализ и его прил. 1988. Т. 22. № 3. С. 15-24.

[54] Sergiescu V. Graphes planaires et présentations des groupes de tresses // Math. Z. 1993. V. 214. P. $477-490$.

[55] Лин В.Я. Косы Артина и связанные с ними группы и пространства // Итоги науки и техники. Алгебра, топология, геометрия 1979. Т. 17. С. 159-227.

[56] Mac Lane S. Categories for the Working Mathematician. New York: Springer-Verlag, 1971. (Graduate Texts in Mathematics.)

[57] Бурбаки Н. Группы и алгебры Ли. Группы Кокстера и системы Титса. Группы, порожденные отражениями. Системы корней. М.: Мир, 1972.

[58] Deligne P. Les immeubles des groupes de tresses généralisés // Invent. Math. 1972. V. 17. P. 273-302.

[59] Брискорн Э. Фундаментальная группа пространства регулярных орбит комплексификации конечной группы, порожденной отражениями // УМН. 1975. Т. 30. №6. С. 147-151.

[60] Chow W.-L. On the algebraical braid group // Ann. of Math. 1948. V. 49. № 3. P. 654-658.

[61] Vershinin V. V. Thom spectra of generalized braid groups // Preprint № 95/02-2. Nantes: Université de Nantes, 1995.

[62] Scott G. P. Braid groups and the group of homeomorphisms of a surface // Proc. Cambridge Philos. Soc. 1970. V. 68. P. 605-617.

[63] Вершинин В. В.О группах кос в телах с ручками // Сиб. матем. журн. 1998. Т. 39. № 4. C. $755-764$

[64] Fenn R., Keyman E., Rourke C. The Singular Braid Monoid Embeds in a Group. Preprint. Brighton: Univ. of Sussex, 1996.

[65] Segal G. B. Classifying spaces and spectral sequences // Publ. Math. I.H.E.S. 1968. V. 34. P. $105-112$

[66] Адамс Дж. Бесконечнократные пространства петель. М.: Мир, 1982. 
[67] May J. P. $E_{\infty}$ spaces, group completions, and permutative categories // London Math. Soc. Lecture Notes Ser. 1974. V. 11. P. 61-93.

[68] Фукс Д. Б. Квилленизация и бордизмы // Функцион. анализ и его прил. 1974. Т. 8. № 1. C. $36-42$.

[69] Waldhausen F. Algebraic $K$-theory of topological spaces. II // Lecture Notes in Math. 1979. V. 763. P. 356-394.

[70] Hatcher A. Homological stability for automorphism groups of free groups // Comment. Math. Helv. 1995. V. 70. P. 39-62.

[71] Segal G. B. Categories and cohomology theories // Topology. 1974. V. 13. P. 293-312.

[72] Cohen F., Taylor L. On the representation theory associated to the cohomology of configuration spaces // Contemp. Math. 1993. V. 146. P. 91-109.

[73] Ossa E. On the cohomology of configuration spaces // Algebraic Topology: New Trends in Localization and Periodicity (Barcelona Conference on Algebraic Topology, 1994). Basel: Birkhäuser, 1996. P. 353-361.

[74] Голубева В.А., Лексин В. П. Квадратичные соотношения в когомологиях обобщенных крашеных кос и тождества Данкла // Функцион. анализ и его прил. 1996. Т. 30. № 2. C. $73-76$.

[75] Greenberg P., Sergiescu V. An acyclic extension of the braid group // Comment. Math. Helv.. V. 66. № 1. P. 109-138.

[76] Dyer E., Lashof R. Homology of iterated loop spaces // Amer. J. Math. 1962. V. 84. № 1. P. $35-88$.

[77] Браун К. С. Когомологии групп. М.: Наука, 1982.

[78] Маркарян Н. С. Гомологии групп кос с нетривиальными коэффициентами // Матем. заметки. 1996. Т. 59. №6. С. 227-231.

[79] Горески М., Макферсон Р. Стратифицированная теория Морса. М.: Мир, 1991.

[80] Orlik P., Solomon L. Combinatorics and topology of complements of hyperplanes // Invent. Math. 1980. V. 56. P. 167-189.

[81] McDuff D. Configuration spaces of positive and negative particles // Topology. 1975. V. 14. P. 91-107.

[82] Bödigheimer C.-F., Cohen F.R., Milgram R. J. Truncated symmetric products and configuration spaces // Math. Z. 1993. V. 214. № 2. P. 179-216.

[83] Joyal A., Street R. Braided tensor categories // Adv. Math. 1993. V. 102. P. 20-78.

[84] Вершинин В. В. Обобщения кос с гомологической точки зрения // Алгебра, геометрия, анализ и матем. физика. 10-я Сибирская школа. Новосибирск: ИМ, 1997. С. 40-62.

[85] Peterson F. P., Toda H. On the structure of $H^{*}(B S F ; Z / p) / /$ J. Math. Kyoto Univ. 1967. V. 7. P. 113-121.

[86] Cohen F., Vershinin V. V. Thom Spectra which are Wedges of Eilenberg-MacLane Spectra // Fields Inst. Commun. 1998. V. 19. P. 43-65.

[87] Dold A., Lashof R. Principal quasifibrations and fiber homotopy equivalence of bundles // Illinois J. Math. 1959. V. 3. P. 285-305.

[88] Мэй Дж. П. Геометрия итерированных пространств петель. Дополнение к книге: Бордман Дж., Фогт Р. Гомотопические инвариантные алгебраические структуры на топологических пространствах. М.: Мир, 1977.

[89] Milgram R. J. The bar construction and abelian H-spaces // Illinois J. Math. 1967. V. 11. P. $242-250$.

[90] Brown E., Peterson F. The stable decomposition of $\Omega^{2} S^{r+2} / /$ Trans. Amer. Math. Soc. 1978. V. 243. P. 287-298.

[91] Cohen R. L. The geometry of $\Omega^{2} S^{3}$ and braid orientations // Invent. Math. 1979. V. 54. P. 53-67.

[92] Brown E., Gitler S. A spectrum whose cohomology is a certain cyclic module over the Steenrod algebra // Topology. 1973. V. 12. P. 283-295.

[93] Bousfield A., Curtis E., Kan D., Quillen D., Rector D., Schlesinger J. The $\bmod p$ lower central series and the Adams spectral sequence // Topology. 1966. V. 5 . P. 331-342. 
[94] Таубс К. Г. Монополи и отображения из $S^{2}$ в $S^{2}$; топология конфигурационного пространства // Монополи. Топологические и вариационные методы. Сб. статей. М.: Мир, 1989. C. $297-379$.

[95] Атья М., Хитчин Н. Геометрия и динамика магнитных монополей. М.: Мир, 1991.

[96] Мэй Дж. П. Теория бесконечнократных пространств петель // УМН. 1981. Т. 36. №6. C. $137-195$.

[97] Thomason R. W. Symmetric monoidal categories model all connective spectra // Theory Appl. Categ. 1995. V. 1. № 5. P. 78-118.

[98] Fiedorowicz Z. Operads and Iterated Monoidal Categories. Preprint. Columbus, OH: Ohio State Univ., 1995.

Институт математики им. С. Л. Соболева, Новосибирск

Поступила в редакцию

E-mail: versh@math.nsc.ru

19.12.1997 